\title{
MODELING AND ANALYZING LARGE SWARMS WITH COVERT LEADERS
}

by

Yu Sun

A dissertation submitted to the Faculty of the University of Delaware in partial fulfillment of the requirements for the degree of Doctor of Philosophy in Applied Mathematics

Spring 2015

(C) $2015 \mathrm{Yu}$ Sun

All Rights Reserved 
ProQuest Number: 3718378

All rights reserved

INFORMATION TO ALL USERS

The quality of this reproduction is dependent upon the quality of the copy submitted.

In the unlikely event that the author did not send a complete manuscript and there are missing pages, these will be noted. Also, if material had to be removed, a note will indicate the deletion.

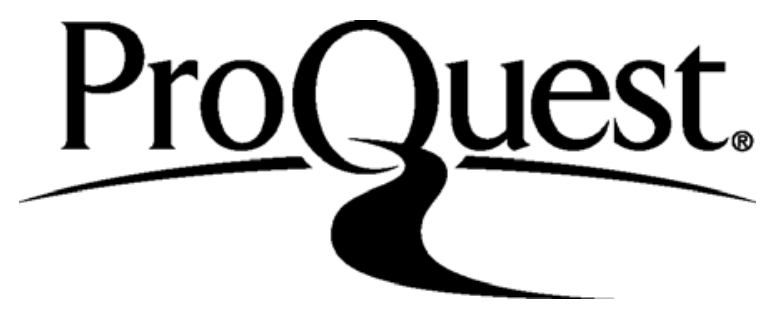

ProQuest 3718378

Published by ProQuest LLC (2015). Copyright of the Dissertation is held by the Author.

All rights reserved.

This work is protected against unauthorized copying under Title 17, United States Code Microform Edition (C) ProQuest LLC.

ProQuest LLC.

789 East Eisenhower Parkway

P.O. Box 1346

Ann Arbor, Ml $48106-1346$ 


\section{MODELING AND ANALYZING LARGE SWARMS WITH COVERT LEADERS}

Yu Sun

Approved:

Louis F. Rossi, Ph.D.

Chair of the Department of Mathematical Sciences

Approved:

George H. Watson, Ph.D.

Dean of the College of Arts \& Sciences

Approved:

James G. Richards, Ph.D.

Vice Provost for Graduate and Professional Education 
I certify that I have read this dissertation and that in my opinion it meets the academic and professional standard required by the University as a dissertation for the degree of Doctor of Philosophy.

Signed:

Louis F. Rossi, Ph.D.

Professor in charge of dissertation

I certify that I have read this dissertation and that in my opinion it meets the academic and professional standard required by the University as a dissertation for the degree of Doctor of Philosophy.

Signed:

John Pelesko, Ph.D.

Member of dissertation committee

I certify that I have read this dissertation and that in my opinion it meets the academic and professional standard required by the University as a dissertation for the degree of Doctor of Philosophy.

Signed:

Pak-Wing Fok, Ph.D.

Member of dissertation committee

I certify that I have read this dissertation and that in my opinion it meets the academic and professional standard required by the University as a dissertation for the degree of Doctor of Philosophy.

Signed:

Chien-Chung Shen, Ph.D.

Member of dissertation committee 


\section{ACKNOWLEDGEMENTS}

First and foremost, I would like to express my special appreciation and thanks to my advisor Professor Dr. Rossi, you have been a tremendous mentor for me. Thank you for encouraging my research and always lighting the light when I was struggling in the dark to find the solution. Without your help, this thesis will not have been started and finished.

I want to thank my external committee member Dr. Chien-Chung Shen. Thank you for spending time on discussing my work every week since I joined the research group and giving me the useful suggestions from the view of a computer scientist.

I would also like to thank my dissertation committee members, Dr. John Pelesko and Dr. Pak-Wing Fok. Thank you for your time to evaluate my research and for your generous help.

I am grateful to the faculty and staff members at the Department of Mathematical Science at University of Delaware. It is my honor to be here for 5 years. Deeply thanks to Dr. Driscoll, Dr. Cakoni, Dr. Hsiao, Dr. Monk, Dr. Colton, Dr. Plechac, Dr. Rakesh, Dr. Leung, Dr. Zhang, Dr. Ou, Dr. Schleiniger, Dr. Cristina Bacuta, Dr. Seraphin, Ms. Deborah See and Ms. Pamela Irwin. You taught me the knowledge and you made my life easier throughout my graduate years.

Thanks to all my friends, you light up my life.

Last but not the least, thanks to my family, especially to my parents. Thank you for your endless love and support. 


\section{TABLE OF CONTENTS}

LIST OF TABLES . . . . . . . . . . . . . . . . . . . v vii

LIST OF FIGURES . . . . . . . . . . . . . . . . . . . . viii

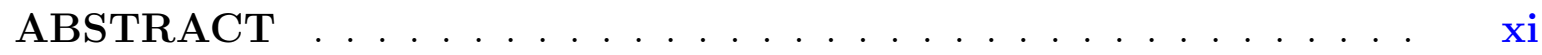

\section{Chapter}

1 THREE-ZONE MODELS OF SWARMS WITH LEADERS . . . . 1

1.1 Collective Animal Behavior Background . . . . . . . . . . . . . . 1

1.2 Introduction to Swarms with Leadership . . . . . . . . . . . . 2

1.3 The Covert Leader Model . . . . . . . . . . . . . . . . . . . . 4

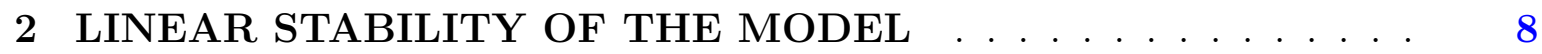

2.1 Linear stability analysis . . . . . . . . . . . . . . . 8

2.1.1 Linearization . . . . . . . . . . . . . . . . . . . . 8

2.1 .2 Analysis . . . . . . . . . . . . . . . . . . . . 14

2.1.3 Conclusion . . . . . . . . . . . . . . . . . . . . . . . 17

2.2 Verification . . . . . . . . . . . . . . . . . . 17

2.3 Conclusion . . . . . . . . . . . . . . . . . . . . . 21

3 COLLECTIVE DECISIONS . . . . . . . . . . . . . . . . . 22

3.1 Experiments . . . . . . . . . . . . . . . . . . . . . . 22

3.2 Mathematical Analysis for the bifurcation . . . . . . . . . . 27

3.2.1 Case study I: Two individuals . . . . . . . . . . . . . 27

3.2.2 Case study II: Extension for two individuals system . . . . . . 37

3.3 Conclusions . . . . . . . . . . . . . . . . . . . . . . . . . 49 
4 INFORMATION TRANSFER IN SWARMS WITH LEADERS

4.1 Introduction . . . . . . . . . . . . . . . . . 50

4.2 Introduction to Information Theory . . . . . . . . . . . . . 51

4.3 Transfer Entropy . . . . . . . . . . . . . . . . . . . . 60

4.4 Local Transfer Entropy . . . . . . . . . . . . . . . . . . . . . 63

4.5 Transfer Entropy for Swarms . . . . . . . . . . . . . . . . . 65

4.6 Results . . . . . . . . . . . . . . . . . . . . 67

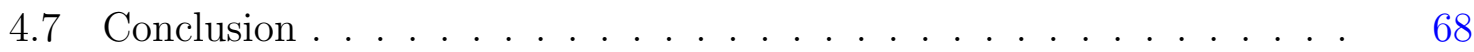

5 USING THE PAGERANK METHOD TO FIND LEADERS IN A

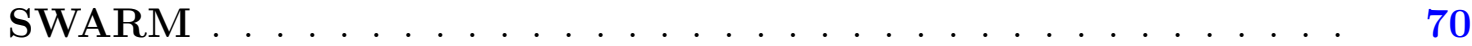

5.1 Introduction to PageRank Method . . . . . . . . . . . . 71

5.2 PageRank Applied to Swarms . . . . . . . . . . . . . . . . . . 75

5.3 Method . . . . . . . . . . . . . . . . . . 76

5.4 Experiments . . . . . . . . . . . . . . . . . . . . . 78

5.4 .1 Couzin Model . . . . . . . . . . . . . . . . . . . . . . 79

5.4.2 The Weight of the External Information . . . . . . . . . . 82

5.4 .3 Our model . . . . . . . . . . . . . . . . . . 85

5.5 Conclusion . . . . . . . . . . . . . . . . . . . 86

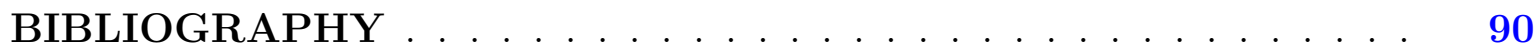




\section{LIST OF TABLES}

1.1 Continuum Swarm Model Variables and parameters . . . . . . . . . 7

$2.1 \quad$ Parameter values . . . . . . . . . . . . . . . . . . . . 18

3.1 Parameter values . . . . . . . . . . . . . . . . 34

$3.2 \quad$ Velocity and position for two individual system . . . . . . . . . 37

3.3 y coordinate for the equilibrium state . . . . . . . . . . 39

3.4 The eigenvalues of $\mathrm{M}$ for $\theta=\pi / 2 \ldots \ldots$. . . . . . . . 44

5.1 Results of PageRank method for finding leaders. 14 experiments are performed with the same parameters except the initial orientation and leaders are randomly chosen. . . . . . . . . . . . . . . 82

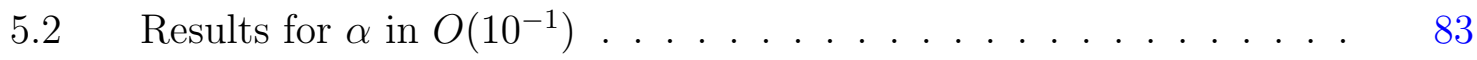

5.3 Results for $\alpha$ in $O\left(10^{-2}\right) \ldots \ldots \ldots$. . . . . . . . . 83 


\section{LIST OF FIGURES}

1.1 ZOR: An individual will try to move away from others in the innermost zone of repulsion. ZOO: An individual will try to align itself with others in the central zone of orientation. ZOA: An individual will try to move toward individuals in the outermost zone of attraction. . . . . . . . . . . . . . . . . .

1.2 Cross-sectional plots of kernels with $\sigma_{1}=3 / 4, \sigma_{2}=9 / 4, \sigma_{3}=15 / 4$. Kernels $G$ and $K$ are scaled so that they can be viewed together with $H \ldots \ldots \ldots \ldots \ldots \ldots \ldots \ldots \ldots$

2.1 In this swarm, $15 \%$ of the individuals are covert leaders using the nonlinear model. The initial velocity is random with the speed varying from 0 to 1 . The leaders possess additional information $\vec{g}=[1,0]^{T}$. Red arrows represent the leaders, and black arrows represent the followers.. . . . . . . . . . . . . . . .

2.2 Final swarm configuration using the QualNet simulator . . . . .

2.3 In this swarm, all parameters are the same as in Figure 3 except we use the linear leadership model. Notice that most of the leaders aggregate at the leading edge.

3.1 Normalized probability of the group direction in an experiment with two groups of leaders. In this case, $7 \%$ of the individuals in the swarm have one type of additional information, and another $7 \%$ have the other type. The $\mathrm{x}$-axis represents difference between the additional information of the two types of leaders. The y-axis represents the direction of the whole group. The color represents the likelihood of the outcome shown on the graph. . . . . . . . . .

3.2 Leaders from one group carrying away the swarm and most of the other leaders. However some leaders splinter away from the swarm.

3.3 The position of the bifurcation point depends on $\rho$. . . . . . . 
3.4 Measurements of the splinter fraction from the experiments described in Figure 3.1. The simulations using ideal interactions and the QualNet simulations are in excellent agreement. . . . . . . . .

3.5 With symmetric initial data, the swarm remains stable even though the symmetry is not enforced as the swarm evolves. . . . . . . .

3.6 Initial condition for the experiments with disjoint leaders . . . . . .

3.7 Random initial velocities but with symmetry imposed along the $\mathrm{x}$

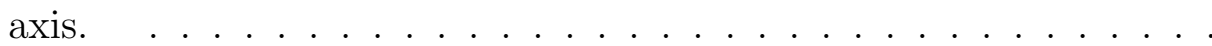

3.8 Splinter fraction from initial conditions on a regular lattice with initial velocity vector $[1,0]^{T} \ldots \ldots$. . . . . . . . . . . 27

3.9 two individuals with different type of external information . . . . $\quad 28$

3.10 The relationship between local interactions and external information at steady state. . . . . . . . . . . . . . . . .

3.11 Graph of $f_{\text {right }}$ in $(-10,0)$. Details of the graph of $f_{\text {right }}$ in $(-2,0)$ are shown on the right . . . . . . . . . . . . . . .

3.12 Weight for external information when $y_{1}$ is in $(-10,0) \ldots . .$. . .

3.13 Interaction for individual 1 when $y_{1}$ is in $(-10,0)$. Detailed plot of interaction for individual 1 when $y_{1}$ is in $(-2,0)$ is shown on the right.

3.14 Bifurcation point for different $\mathrm{m} \ldots$. . . . . . . . . . . . 46

3.15 Refined Bifurcation point for different $\mathrm{m}$. . . . . . . . . . . 47

3.16 In this swarm, both of them are leaders containing opposite external information, they finally get to a stable steady state. . . . . . . . . 48

3.17 In this swarm, both of them are leaders containing different external information $\theta=6 \pi / 18$ and they finally get to a stable steady state.

3.18 In this swarm, both of them are leaders containing opposite external information, and they finally split. . . . . . . . . . . . . 49

$4.1 \quad H(p)$ versus $\mathrm{p} \ldots \ldots \ldots \ldots \ldots \ldots$ 
4.2 The transfer entropy received by followers and leaders over time along with the swarm configuration at key times during self-organization. Leaders are displayed in red, but have the same interaction influence others no differently from the followers. . . . . . . . . . . . .

$5.1 \quad$ Linked pages . . . . . . . . . . . . . . . . 71

5.2 The process of creating binary data for individual $i \ldots$. . . . . . 77

5.3 Movement at key time step . . . . . . . . . . . . . . 80

$5.4 \quad$ Leader's index position in the sorted eigenvector . . . . . . . . . . . 81

5.5 Leader's index position in the sorted eigenvector . . . . . . . . . . . 81

$5.6 \quad$ Leader's index position in the sorted eigenvector for $\alpha=0.009 \ldots 3$

$5.7 \quad$ Leader's index position in the sorted eigenvector for $\alpha=0.001$. . 84

5.8 Leader's index position in the 40 smallest entires of the sorted

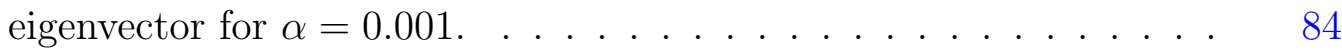

$5.9 \quad$ Leader's index position in the sorted eigenvector for $\alpha=0.0005$. . . 85

5.10 Leader's index position in the sorted eigenvector for our model. . . 86

5.11 Weight before the external information for leaders along the time . 86

5.12 Leader 49's movement at key time step . . . . . . . . . . . . 87 


\begin{abstract}
Swarm dynamics is the study of collections of agents that interact with one another without central control. In natural systems, insects, birds, fish and other large mammals function in larger units to increase the overall fitness of the individuals. Their behavior is coordinated through local interactions to enhance mate selection, predator detection, migratory route identification and so forth [3, 7, 23, 28, 35]. Some of the individuals may possess additional information, like food sources. Those individuals with additional information may act as leaders and affect the movement of the whole group. In artificial systems, swarms of automated devices can augment human activities such as search and rescue, and environmental monitoring by covering large areas with multiple nodes $[2,8,26,27,34]$. Sometimes we would like to inject additional information into the system without telling every device. Then the informed one functions as a leader. We would like to build a model to simulate the behavior of swarms with covert leaders. Then based on the model, we would like to know the stability of the system, the collective decision of the swarms when there is knowledge confliction, and the way to find the covert leaders when we observe a group of individuals in motion.

In Chapter 1, we extend the covert leadership model in large swarms. A leader is a member of the swarm that acts upon information in addition to what is provided by local interactions. A covert leader is a leader that is treated no differently than others in the swarm, so leaders and followers participate equally in whatever interaction model is used [32]. We focus our efforts on the behaviors driven by the three-zone swarming model and present a new nonlinear model in which leaders will respond more strongly to additional information when the swarm is less dense. Similarly, leaders in dense regions behave more like followers.
\end{abstract}


In Chapter 2, we perform linear stability analysis on the model. The result is the same as the leaderless model, which says that the growth or decay of perturbations in an infinite, uniform swarm depends on the strength of attraction relative to repulsion and orientation. It tells us that we could inject additional information into the system without changing the stability criteria. We verify our analysis with simulation. We also compare our model with more popular linear leadership models. The leaders in our model are embedded in the swarms instead of accumulating into the front in contrast to the linear model. We apply this model to wireless robotic applications, in which densities are calculated utilizing positions of neighboring robots. The result on the QualNet platform is consistent with our ideal simulation results.

In Chapter 3, we explore problems where two classes of covert leaders with different information try to influence the same swarm. The swarms will choose the average direction if the information differential is small. The swarms will randomly choose a direction of the leaders' if the information differential is large. We validate our modeling and analysis using realistic wireless protocols and channel models on the QualNet network simulator. We also perform two case studies which are simplified forms of our model to find the bifurcation point analytically.

In Chapter 4, we try to solve the problem: whether or not it is possible to distinguish between followers and leaders when we observe a group of individuals in motion. We explore the interplay between swarm dynamics, covert leadership and theoretical information transfer. Depending upon the leadership model, leaders can use their external information either all the time or in response to local conditions [10, 37]. We use theoretical information transfer as a means of analyzing swarm interactions. We find that covert leaders can be distinguished from followers in a swarm because they receive less transfer entropy than followers.

Finally, in Chapter 5, we would like to find a method to detect who are the leaders and who are the followers. Inspired by the PageRank method which is used by Google to rank the importance of web pages, we apply a modified PageRank method to the swarms. We test this method on the Couzin model so that we could control 
the weight of the external information that the leaders respond to. We find that the method works well when the leaders respond to the external information relatively strongly which means the weight of the external information that the leaders respond to should be above $O\left(10^{-2}\right)$. To our nonlinear model, the weight is changing with time and below $O\left(10^{-2}\right)$. This method can not detect the leaders in our model. From this point of view, the leaders in our model really are covert. 


\section{Chapter 1}

\section{THREE-ZONE MODELS OF SWARMS WITH LEADERS}

\subsection{Collective Animal Behavior Background}

Living in groups is a widespread phenomena in the world of animals, such as schools of fish, flocks of birds and herds of sheep. Being a member of a group could gain benefits in many ways [36]. Weimerskirch et al. [43] provide empirical evidence showing that the great white pelicans trained to fly in ' $\mathrm{V}$ ' formation save a significant amount of energy measured through the heart rates, especially for the followers, which enables the pelicans to increase their foraging or migratory range. Animals can also conserve heat and water by huddling together, due to the reduced surface area and the increased temperature or humidity of the surrounding air [16]. Access to information is another key benefit of being near to others. The ocean skater could initiate avoidance behavior before an approaching predator can be seen due to the interactions between individuals, in the form of increased bodily encounters and/or visual stimulation by adjacent individuals [38]. This type of information transfer is based on cues. Information could also be transferred through other signals. For example, many species of ants deposit pheromones when they return to the nest after finding a food souce. Other ants will follow the trail and find the food when they encounter those trails [44].

Often when animals aggregate together, density patterns emerge. A large wildebeest herd, viewed from the above, migrates in a common direction of motion, and ex-

hibits a wavelike broad front [9]. Fish schools also come in many different shapes and sizes: stationary swarms; predator avoiding vacuoles and flash expansions; hourglasses and vortices; highly aligned cruising parabolas, herds, and balls [36]. The formation of the group level pattern is said to be self-organized because it is not encoded in 
the individual level rules [36]. Finding the simplest explanation for complex collective phenomena becomes one of the principal aims of self-organization theory [9].

Mathematical modeling is a good way to solve the mystery of collective behavior. Niwa presents a model that could predict group size distribution and the results hold for various data from pelagic fishes and mammalian herbivores in the wild [24]. Later, he shows another work describing the dynamics of groups of individuals on a constrained lattice [25]. It extends the non-spatial model introduced in [24], but the limitation of this work is that it does not describe how the interactions between individuals produce the group dynamics. Self-propelled particle (SPP) models give a possible way to solve such kinds of problem. The concept of SPP was first introduced in 1995 by Vicsek et al [39], and has been used by many investigators [20, 4, 11, 14, 19, 31, 39, 42]. The particles in the SPP model move in one-, two-, or three-dimensions. Each particle has a local interaction zone within which it responds to other particles. The exact form of the interactions varies between models, but in general, it contains attraction, and/or alignment, and/or repulsion.

\subsection{Introduction to Swarms with Leadership}

This thesis mainly focuses on modeling and analyzing large swarms with leadership. In natural systems, animals that forage or travel in groups, make movement decisions that depend on social interactions among group members. However, in many cases, a small number of individuals have pertinent information, such as knowledge about the location of a food source or a migration route [10]. Here we just consider the case that the information is transferred by local interactions or cues but not signals. Then those individuals possessing the additional information will act as covert leaders. A leader is an individual guided by additional information that ordinary individuals, followers, do not possess. A covert leader is a leader who acts on additional information but is treated like all the other individuals in the swarm [32]. More precisely, all swarm influences must be functions of the sum of the covert leaders and followers taken together because it is not possible to distinguish one from the other. There are 
long-standing conjectures about the nature of information transfer in swarms through mutual interactions. Wang et. al.[40] recently established for the first time direct evidence of information cascades in swarms using a three-zone model without the influence of leadership in the case where two or more coherent structures interact with one another. Leadership provides a way to inject additional information directly into a swarm without additional environment cues.

For the purposes of this study, we limit our consideration to three-zone swarming, a special case in SPP models. In three-zone swarming behavior, individual behavior is driven by the position and orientation of neighboring individuals in each of the three concentric zones. An individual will try to move away from others in the innermost zone of repulsion. An individual will try to align itself with others in the central zone of orientation. Finally, an individual will try to move toward individuals in the outermost zone of attraction. Individual behavior is the weighted contribution from the three influences.

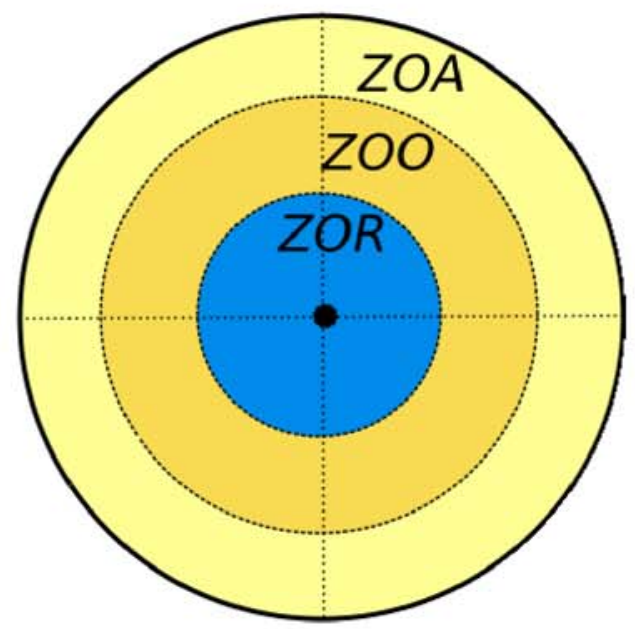

Figure 1.1: ZOR: An individual will try to move away from others in the innermost zone of repulsion. ZOO: An individual will try to align itself with others in the central zone of orientation. ZOA: An individual will try to move toward individuals in the outermost zone of attraction.

We will explore the dynamics of large swarms by treating the continuum limit of the underlying model. In this limit, individuals are represented as a density which is a function of space and time. Similarly, the velocities of discrete individuals are 
represented by a velocity function. The dynamics of the swarm is described by a system of coupled partial differential equations capturing necessary conservation principles and the local interactions (i.e. behavior) between neighboring members of the swarm.

\subsection{The Covert Leader Model}

Animals do not need long-range information to coordinate group behavior, a fact which is often stressed by proponents of self-organization theory. They also state that localizing information input may provide significant adaptive benefits to an individual within a group, allowing sensitive response not only to predators but also to environmental obstacles [9]. When developing our continuum model, we assume that the individuals in a swarm make decisions based only on the positions and velocities of nearby individuals rather than using information about every member of the swarm. We use the same Gaussians kernels (and moments of Gaussians) used by Miller et. al. that give the desired effects of repulsion, orientation, and attraction [20].

$$
\begin{array}{r}
H_{\sigma_{1}}=\frac{1}{8 \pi \sigma_{1}^{4}} \vec{x} \exp \left(-\frac{|\vec{x}|^{2}}{4 \sigma_{1}^{2}}\right) \quad \text { (repulsion) } \\
G_{\sigma_{2}}=\frac{1}{4 \pi \sigma_{2}^{2}} \exp \left(-\frac{|\vec{x}|^{2}}{4 \sigma_{2}^{2}}\right) \quad \text { (orientation) } \\
K_{\sigma_{3}}=-\frac{1}{64 \pi \sigma_{3}^{6}} \vec{x}|\vec{x}|^{2} \exp \left(-\frac{|\vec{x}|^{2}}{4 \sigma_{3}^{2}}\right) \quad \text { (attraction) }
\end{array}
$$

The parameters $\sigma_{1}, \sigma_{2}, \sigma_{3}$ are the sizes of the zones. These zones will overlap because they are continuous with infinite support. However, they decay exponentially so that there are distinct zones where one kernel dominates the interaction over the other two. The kernels are normalized to preserve density or density gradients and therefore make the swarming model self-consistent. Specifically, if we apply test functions,

$$
\begin{aligned}
& \phi(\vec{x})=\vec{a} \cdot \vec{x}+b, \\
& \psi(\vec{x})=b,
\end{aligned}
$$


then

$$
\begin{aligned}
& H_{\sigma_{1}} * \phi=-\vec{a}, \\
& G_{\sigma_{2}} * \psi=b . \\
& K_{\sigma_{3}} * \phi=\vec{a},
\end{aligned}
$$

The convolution is performed on the whole domain from $-\infty$ to $\infty$.

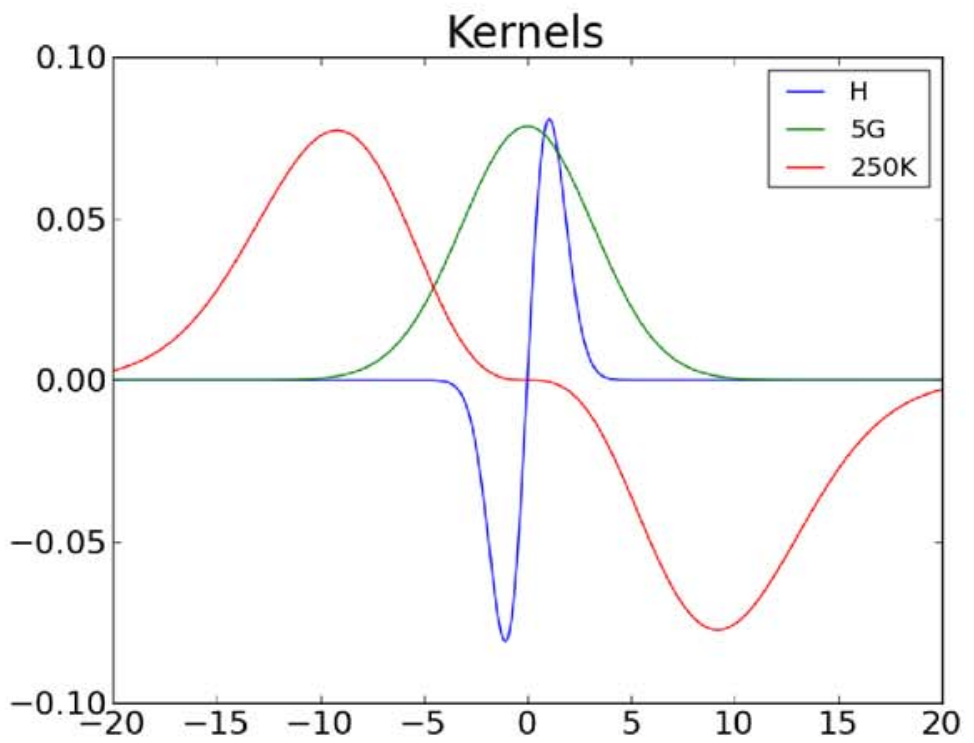

Figure 1.2: Cross-sectional plots of kernels with $\sigma_{1}=3 / 4, \sigma_{2}=9 / 4, \sigma_{3}=15 / 4$. Kernels $G$ and $K$ are scaled so that they can be viewed together with $H$.

Using the same methodology as in [20], we derive evolution equations based on conservation principles and behavioral principles for a swarm with covert leaders.

The swarm must conserve mass because followers and leaders are neither created nor destroyed.

$$
\begin{aligned}
& \frac{\partial \rho_{f}}{\partial t}+\nabla \cdot\left(\rho_{f} \vec{v}_{f}\right)=0 \\
& \frac{\partial \rho_{l}}{\partial t}+\nabla \cdot\left(\rho_{l} \vec{v}_{l}\right)=0
\end{aligned}
$$


The desired velocity of the followers is made through local interactions (repulsion, orientation, attraction):

$$
\vec{v}_{f d}=H_{\sigma_{1}} *\left(\rho_{f}+\rho_{l}\right)+\frac{G_{\sigma_{2}} *\left(\rho_{f} \vec{v}_{f}+\rho_{l} \vec{v}_{l}\right)}{G_{\sigma_{2}} *\left(\rho_{f}+\rho_{l}\right)}+c_{a} K_{\sigma_{3}} *\left(\rho_{f}+\rho_{l}\right)
$$

Where $H_{\sigma_{1}} *\left(\rho_{f}+\rho_{l}\right)$ represents repulsion, $\frac{G_{\sigma_{2}} *\left(\rho_{f} \vec{v}_{f}+\rho_{l} \vec{v}_{l}\right)}{G_{\sigma_{2}} *\left(\rho_{f}+\rho_{l}\right)}$ represents orientation, and $K_{\sigma_{3}} *\left(\rho_{f}+\rho_{l}\right)$ represents attraction.

Covert leaders respond to a combination of social cues, similar to leaderless swarming, and a preferred velocity vector known to the covert leader but not the followers. Gordon et al. showed that the contact rates increase when density was low and decrease when density was high [13]. Here we present a new nonlinear model in which a leader will respond more strongly to additional information when the swarm is less dense, meaning there are fewer individuals with which to interact. Conversely, leaders in denser regions behave more like followers. The relative importance of social cues to the known preferred velocity depends exponentially on the local density. The parameter $\rho$ is a density over which the influence of the known velocity will decay by a factor of $1 / e$. Decreasing $\rho$ makes leaders more sensitive to density changes.

$$
\begin{aligned}
\vec{v}_{l d}= & {\left[1-e^{-\frac{G_{\sigma_{2}} *\left(\rho_{f}+\rho_{l}\right)}{\rho}}\right]\left[H_{\sigma_{1}} *\left(\rho_{f}+\rho_{l}\right)+\frac{G_{\sigma_{2}} *\left(\rho_{f} \vec{v}_{f}+\rho_{l} \vec{v}_{l}\right)}{G_{\sigma_{2}} *\left(\rho_{f}+\rho_{l}\right)}\right.} \\
& \left.+c_{a} K_{\sigma_{3}} *\left(\rho_{f}+\rho_{l}\right)\right]+e^{-\frac{G_{\sigma_{2}} *\left(\rho_{f}+\rho_{l}\right)}{\rho}} \vec{g}
\end{aligned}
$$

where $\vec{g}$ represents the external information. Once the desired velocity has been determined, the velocity changes toward $\vec{v}_{l d}$ and $\vec{v}_{f d}$ by a linear control process:

$$
\begin{aligned}
& \frac{\partial \vec{v}_{f}}{\partial t}+\left(\vec{v}_{f} \cdot \nabla\right) \vec{v}_{f}=\kappa\left(\vec{v}_{f d}-\vec{v}_{f}\right) \\
& \frac{\partial \vec{v}_{l}}{\partial t}+\left(\vec{v}_{l} \cdot \nabla\right) \vec{v}_{l}=\kappa\left(\vec{v}_{l d}-\vec{v}_{l}\right)
\end{aligned}
$$

The meaning of the parameters or variables in the model are shown in Table 1.1.

Using this concise mathematical approach, one can search for stable structures in large swarms and determine how they depend upon size and influence of each of the zones. Earlier results [20] explain the emergence of specific axisymmetric and nonaxisymmetric structures in swarms without leaders. In addition, we could explain how 
Table 1.1: Continuum Swarm Model Variables and parameters

\begin{tabular}{|c|c|}
\hline \hline$t$ & Time \\
\hline$\vec{x}$ & Position \\
\hline$\rho_{f}(\vec{x}, t)$ & Follower density \\
\hline$\rho_{l}(\vec{x}, t)$ & Leader density \\
\hline$\vec{v}_{f}(\vec{x}, t)$ & Follower velocity \\
\hline$\vec{v}_{l}(\vec{x}, t)$ & Leader velocity \\
\hline$\vec{v}_{f d}(\vec{x}, t)$ & Followers' desired direction \\
\hline$\vec{v}_{l d}(\vec{x}, t)$ & Leaders' desired direction \\
\hline$\kappa$ & Turning/acceleration rate \\
\hline$c_{a}$ & Attraction/repulsion ratio \\
\hline$\vec{g}$ & $\begin{array}{c}\text { Additional information (direction) } \\
\text { known only to leaders }\end{array}$ \\
\hline \hline
\end{tabular}

smaller compact structures are preferred over larger structures given certain parameter combinations.

These results also apply to traditional leadership models in which leaders' behaviors are a linear combination of swarm interaction terms and additional information. However, in these regimes, leaders are influenced by additional information independent of nearby individuals. For the models we present here, since the response is slightly nonlinear, new stability criteria and new structures emerge as a function of swarming parameters. We will discuss the new stability criteria and new structure in Chapter 2. 


\section{Chapter 2 \\ LINEAR STABILITY OF THE MODEL}

\subsection{Linear stability analysis}

The environment may affect the behavior of the swarm. Radakov used an artificial stimulus to frighten only a small part of a school of silverside fish. He observed that the fish nearest to the stimulus change their directions away from the stimulus immediately and then this behavior propagates to the whole group [30]. In this experiment, the small disturbance has been amplified by the swarm. But we would like to know whether the disturbance would damp out under special conditions.

We want to predict the group's behavior without simulating all possible environmental factors. Linear stability analysis gives us a way to analyze a dynamic system. We firstly linearize the system around a steady state. And then we can examine the response of the system to see whether the perturbation will damp out or be amplified. This result will indicate the general behavior of the group.

For our system, an infinite swarm with uniform density moves in the direction dictated by the leaders: $\vec{g}=\vec{v}^{0}$ is an equilibrium solution. We want to understand the effect of a small disturbance in the densities and velocities in our system. So we

linearize the equations, add plane waves which have terms proportional to $e^{i(\vec{\xi} \vec{x}-\omega t)}$ and analyze the dispersion relation $\omega(\vec{\xi})$. When the imaginary part of $\omega$ is positive, the perturbation will grow, and when the imaginary part of $\omega$ is negative, the perturbation will damp out. Our analysis is done in 2D.

\subsubsection{Linearization}

At steady state, all the agents regardless of whether they are leaders or followers should have the same velocity. We use $\vec{v}^{0}$ to represent the velocity of the swarm. This 
value should be equal to the external information denoted by $\vec{g}$. Then the desired velocity of the leader $\vec{v}_{l d}$ and the desired velocity of the follower $\vec{v}_{f d}$ should also be equal to the current velocity so that the velocity of the individuals will not change. Mathematically we could represent the relationship among the desired velocity, current velocity and external information at steady state as follows:

$$
\vec{v}_{f}^{0}=\vec{v}_{l}^{0}=\vec{v}_{f d}^{0}=\vec{v}_{l d}^{0}=\vec{g}=\vec{v}^{0}
$$

where $\vec{v}_{f}^{0}$ and $\vec{v}_{l}^{0}$ represent the current velocity of the leaders and the followers.

We use $\rho_{f}^{0}$ to represent the density of the followers, and $\rho_{l}^{0}$ to represent the density of the leaders at steady state. At steady state, we assume that the swarms are uniformly distributed and have the following density:

$$
\rho_{f}^{0}+\rho_{l}^{0}=1
$$

We add a small perturbation to the density $\rho_{f}^{0}, \rho_{l}^{0}$ and velocity $\vec{v}_{l}^{0}, \vec{v}_{f}^{0}$ with $O(\epsilon)$, where $\epsilon$ is a small number.

$$
\begin{aligned}
\rho_{f} & =\rho_{f}^{0}+\epsilon \rho_{f}^{1}+\cdots & \rho_{l} & =\rho_{l}^{0}+\epsilon \rho_{l}^{1}+\cdots \\
\vec{v}_{f} & =\vec{v}_{f}^{0}+\epsilon \vec{v}_{f}^{1}+\cdots & \vec{v}_{l} & =\vec{v}_{l}^{0}+\epsilon \vec{v}_{l}^{1}+\cdots \\
\vec{v}_{f d} & =\vec{v}_{f d}^{0}+\epsilon \vec{v}_{f d}^{1}+\cdots & \vec{v}_{l d} & =\vec{v}_{l d}^{0}+\epsilon \vec{v}_{l d}^{1}+\cdots
\end{aligned}
$$


Then we linearize the PDEs by expanding about $\epsilon$ and collecting terms of $O(\epsilon)$.

$$
\begin{aligned}
& \vec{v}_{f d}^{1}=H_{\sigma_{1}} *\left(\rho_{f}^{1}+\rho_{l}^{1}\right)+G_{\sigma_{2}} *\left(\rho_{f}^{0} \vec{v}_{f}^{1}+\rho_{l}^{0} \vec{v}_{l}^{1}\right)+c_{a} K_{\sigma_{3}} *\left(\rho_{f}^{1}+\rho_{l}^{1}\right) \\
& \quad \begin{aligned}
\vec{v}_{l d}^{1}= & \left(1-e^{-\frac{1}{\rho}}\right)\left(H_{\sigma_{1}} *\left(\rho_{f}^{1}+\rho_{l}^{1}\right)+G_{\sigma_{2}} *\left(\rho_{f}^{0} \vec{v}_{f}^{1}+\rho_{l}^{0} \vec{v}_{l}^{1}\right)\right. \\
& \left.+c_{a} K_{\sigma_{3}} *\left(\rho_{f}^{1}+\rho_{l}^{1}\right)\right)
\end{aligned} \\
& \frac{\partial \vec{v}_{f}^{1}}{\partial t}+\left(\vec{v}^{0} \cdot \nabla\right) \vec{v}_{f}^{1}=\kappa\left(\vec{v}_{f d}^{1}-\vec{v}_{f}^{1}\right) \\
& \frac{\partial \vec{v}_{l}^{1}}{\partial t}+\left(\vec{v}^{0} \cdot \nabla\right) \vec{v}_{l}^{1}=\kappa\left(\vec{v}_{l d}^{1}-\vec{v}_{l}^{1}\right) \\
& \frac{\partial \rho_{f}^{1}}{\partial t}+\nabla \rho_{f}^{1} \cdot \vec{v}^{0}+\rho_{f}^{0}\left(\nabla \cdot \vec{v}_{f}^{1}\right)=0 \\
& \frac{\partial \rho_{l}^{1}}{\partial t}+\nabla \rho_{l}^{1} \cdot \vec{v}^{0}+\rho_{l}^{0}\left(\nabla \cdot \vec{v}_{l}^{1}\right)=0
\end{aligned}
$$

The first four equations can be reduced to two by plugging in the expressions for $\vec{v}_{f d}^{1}$ and $\vec{v}_{l d}^{1}$ given by (2.1a) and (2.1b) into (2.1c) and (2.1d),

$$
\begin{aligned}
& \frac{\partial \vec{v}_{f}^{1}}{\partial t}+\left(\vec{v}^{0} \cdot \nabla\right) \vec{v}_{f}^{1}= \kappa\left(H_{\sigma_{1}} *\left(\rho_{f}^{1}+\rho_{l}^{1}\right)+G_{\sigma_{2}} *\left(\rho_{f}^{0} \vec{v}_{f}^{1}+\rho_{l}^{0} \vec{v}_{l}^{1}\right)\right. \\
&\left.+c_{a} K_{\sigma_{3}} *\left(\rho_{f}^{1}+\rho_{l}^{1}\right)-\vec{v}_{f}^{1}\right) \\
& \frac{\partial \vec{v}_{l}^{1}}{\partial t}+\left(\vec{v}^{0} \cdot \nabla\right) \vec{v}_{l}^{1}= \kappa\left(( 1 - e ^ { - \frac { 1 } { \rho } } ) \left(H_{\sigma_{1}} *\left(\rho_{f}^{1}+\rho_{l}^{1}\right)+G_{\sigma_{2}} *\left(\rho_{f}^{0} \vec{v}_{f}^{1}+\rho_{l}^{0} \vec{v}_{l}^{1}\right)\right.\right. \\
&\left.\left.+c_{a} K_{\sigma_{3}} *\left(\rho_{f}^{1}+\rho_{l}^{1}\right)\right)-\vec{v}_{l}^{1}\right) \\
& \frac{\partial \rho_{f}^{1}}{\partial t}+\nabla \rho_{f}^{1} \cdot \vec{v}^{0}+\rho_{f}^{0}\left(\nabla \cdot \vec{v}_{f}^{1}\right)=0 \\
& \frac{\partial \rho_{l}^{1}}{\partial t}+\nabla \rho_{l}^{1} \cdot \vec{v}^{0}+\rho_{l}^{0}\left(\nabla \cdot \vec{v}_{l}^{1}\right)=0
\end{aligned}
$$

Our analysis of the plane waves will take place in Fourier space.

$$
\hat{f}(\xi)=\int_{-\infty}^{\infty} f(x) e^{i \xi \cdot x} d x
$$


The transformed equations for (2.2) are:

$$
\begin{aligned}
& \left(\hat{v_{f}^{1}}\right)_{t}-i\left(\vec{v}^{0} \cdot \xi\right) \hat{v}_{f}^{1}=\kappa\left(\left(\hat{H}_{\sigma_{1}}+c_{a} \hat{K_{\sigma_{3}}}\right)\left(\hat{\rho_{f}^{1}}+\hat{\rho_{l}^{1}}\right)+\hat{G_{\sigma_{2}}}\left(\rho_{f}^{0} \hat{v_{f}^{1}}+\rho_{l}^{0} \hat{v_{l}^{1}}\right)-\hat{v_{l}^{1}}\right) \\
& \left(\hat{v_{l}^{1}}\right)_{t}-i\left(\vec{v}^{0} \cdot \xi\right) \hat{v_{l}^{1}}=\kappa\left(\left(1-\hat{e}^{-\frac{1}{\rho}}\right)\left(\left(\hat{H}_{\sigma_{1}}+c_{a} \hat{K_{\sigma_{3}}}\right)\left(\hat{\rho_{f}^{1}}+\hat{\rho_{l}^{1}}\right)+\hat{G_{\sigma_{2}}}\left(\hat{\rho_{f}^{0}} \hat{v_{f}^{1}}+\hat{\rho_{l}^{0}} \hat{v_{l}^{1}}\right)\right)-\hat{v_{l}^{1}}\right) \\
& \quad \frac{\partial \hat{\rho_{f}^{1}}}{\partial t}-i \hat{\rho_{f}^{1}}\left(\xi \cdot \vec{v}^{0}\right)-i \rho_{f}^{0}\left(\xi \cdot \hat{v_{f}^{1}}\right)=0 \\
& \frac{\partial \hat{\rho_{l}^{1}}}{\partial t}-i \hat{\rho}_{l}^{1}\left(\xi \cdot \vec{v}^{0}\right)-i \rho_{l}^{0}\left(\xi \cdot \hat{v_{l}^{1}}\right)=0
\end{aligned}
$$

The transformed kernels are:

$$
\begin{aligned}
& \widehat{H_{\sigma_{1}}}=i \vec{\xi} \exp \left(-\sigma_{1}^{2}|\vec{\xi}|^{2}\right) \\
& \widehat{G_{\sigma_{2}}}=\exp \left(-\sigma_{2}^{2}|\vec{\xi}|^{2}\right) \\
& \widehat{K_{\sigma_{3}}}=\frac{1}{2} i\left(-2+\sigma_{3}^{2}|\vec{\xi}|^{2}\right) \vec{\xi} \exp \left(-\sigma_{3}^{2}|\vec{\xi}|^{2}\right)
\end{aligned}
$$

We substitute plane waves (or their Fourier transforms) into the linearized equations.

$$
\begin{aligned}
& \rho_{f}^{1}=A e^{i\left(x \xi_{0}-\omega t\right)} \quad \widehat{\rho_{f}^{1}}=4 \pi A \delta\left(\xi-\xi_{0}\right) e^{-i \omega t} \\
& \rho_{l}^{1}=B e^{i\left(x \xi_{0}-\omega t\right)} \quad \widehat{\rho_{l}^{1}}=4 \pi B \delta\left(\xi-\xi_{0}\right) e^{-i \omega t} \\
& \vec{v}_{f}^{1}=\vec{C} e^{i\left(x \xi_{0}-\omega t\right)} \quad \widehat{v_{f}^{1}}=4 \pi \vec{C} \delta\left(\xi-\xi_{0}\right) e^{-i \omega t} \\
& \vec{v}_{l}^{1}=\vec{D} e^{i\left(x \xi_{0}-\omega t\right)} \quad \widehat{v_{l}^{1}}=4 \pi \vec{D} \delta\left(\xi-\xi_{0}\right) e^{-i \omega t}
\end{aligned}
$$

Since the velocities are vector-valued, the coefficients C and D are vectors.

$$
\begin{aligned}
& \vec{C}=\left[\begin{array}{l}
C_{1} \\
C_{2}
\end{array}\right] \\
& \vec{D}=\left[\begin{array}{c}
D_{1} \\
D_{2}
\end{array}\right]
\end{aligned}
$$


Simplifying,

$$
\begin{aligned}
& A \omega+A\left(\vec{\xi}_{0} \cdot \vec{v}_{0}\right)+\rho_{f}^{0}\left(\vec{\xi}_{0} \cdot \vec{C}\right)=0 \\
& B \omega+B\left(\vec{\xi}_{0} \cdot \vec{v}_{0}\right)+\rho_{l}^{0}\left(\overrightarrow{\xi_{0}} \cdot \vec{D}\right)=0 \\
& -\vec{C} i \omega-\vec{C} i\left(\vec{\xi}_{0} \cdot \vec{v}_{0}\right)=\kappa\left(\left(\hat{H_{\sigma_{1}}}+c_{a} \hat{K_{\sigma_{3}}}\right)(A+B)+\hat{G_{\sigma_{2}}}\left(\vec{C} \rho_{f}^{0}+\vec{D} \rho_{l}^{0}\right)-\vec{C}\right) \\
& -\vec{D} i \omega-\vec{D} i\left(\vec{\xi}_{0} \cdot \vec{v}_{0}\right)=\kappa\left(\left(1-e^{-\frac{1}{\rho}}\right)\left(\left(\hat{H_{\sigma_{1}}}+c_{a} \hat{K_{\sigma_{3}}}\right)(A+B)+\hat{G_{\sigma_{2}}}\left(\vec{C} \rho_{f}^{0}+\vec{D} \rho_{l}^{0}\right)\right)-\vec{D}\right)
\end{aligned}
$$

Separating $(2.5 \mathrm{c})$ and $(2.5 \mathrm{~d})$ each into two equations and breaking the vectors into components, $\widehat{H_{\sigma_{1}}}$ and $\widehat{K_{\sigma_{3}}}$ are both vector quantities represented by

$$
\widehat{H_{\sigma_{1}}}=\left(\begin{array}{c}
\hat{H}_{1} \\
\hat{H}_{2}
\end{array}\right) \quad \widehat{K_{\sigma_{3}}}=\left(\begin{array}{c}
\hat{K}_{1} \\
\hat{K}_{2}
\end{array}\right)
$$

and

$$
\hat{\xi_{0}}=\left(\begin{array}{c}
\xi_{1} \\
\xi_{2}
\end{array}\right) \quad \hat{v}_{0}=\left(\begin{array}{c}
v_{1} \\
v_{2}
\end{array}\right)
$$

Now we have a set of 6 equations and 6 unknowns $\left(A, B, C_{1}, C_{2}, D_{1}, D_{2}\right)$. For this system, we could write it into a matrix form.

$$
\begin{aligned}
& A\left(\omega+\overrightarrow{\xi_{0}} \cdot \overrightarrow{v_{0}}\right)+C_{1}\left(\rho_{f}^{0} \xi_{1}\right)+C_{2}\left(\rho_{f}^{0} \xi_{2}\right)=0 \\
& B\left(\omega+\overrightarrow{\xi_{0}} \cdot \vec{v}_{0}\right)+D_{1}\left(\rho_{l}^{0} \xi_{1}\right)+D_{2}\left(\rho_{l}^{0} \xi_{2}\right)=0 \\
& A\left(\kappa\left(\hat{H}_{1}+c_{a} \hat{K_{1}}\right)\right)+B\left(\kappa\left(\hat{H}_{1}+c_{a} \hat{K_{1}}\right)\right)+ \\
& C_{1}\left(i \omega+i\left(\overrightarrow{\xi_{0}} \cdot \vec{v}_{0}\right)-\kappa+\kappa \rho_{f}^{0} \hat{G_{\sigma_{2}}}\right)+D_{1}\left(\kappa \rho_{l}^{0} \hat{G_{\sigma_{2}}}\right)=0 \\
& A\left(\kappa\left(\hat{H_{2}}+c_{a} \hat{K_{2}}\right)\right)+B\left(\kappa\left(\hat{H_{2}}+c_{a} \hat{K_{2}}\right)\right)+ \\
& C_{2}\left(i \omega+i\left(\overrightarrow{\xi_{0}} \cdot \vec{v}_{0}\right)-\kappa+\kappa \rho_{f}^{0} \hat{G_{\sigma_{2}}}\right)+D_{2}\left(\kappa \rho_{l}^{0} \hat{G_{\sigma_{2}}}\right)=0 \\
& A \kappa\left(\left(1-e^{-\frac{1}{\rho}}\right)\left(\hat{H_{1}}+c_{a} \hat{K_{1}}\right)\right)+B \kappa\left(\left(1-e^{-\frac{1}{\rho}}\right)\left(\hat{H_{1}}+c_{a} \hat{K_{1}}\right)\right)+ \\
& C_{1}\left(1-e^{-\frac{1}{\rho}}\right) \kappa \rho_{f}^{0} \hat{G_{\sigma_{2}}}+D_{1}\left(i \omega+i\left(\vec{\xi}_{0} \cdot \vec{v}_{0}\right)-\kappa+\left(1-e^{-\frac{1}{\rho}}\right) \kappa \rho_{l}^{0} \hat{G_{\sigma_{2}}}\right)=0 \\
& A \kappa\left(\left(1-e^{-\frac{1}{\rho}}\right)\left(\hat{H_{2}}+c_{a} \hat{K_{2}}\right)\right)+B \kappa\left(\left(1-e^{-\frac{1}{\rho}}\right)\left(\hat{H_{2}}+c_{a} \hat{K_{2}}\right)\right)+ \\
& C_{2}\left(1-e^{-\frac{1}{\rho}}\right) \kappa \rho_{f}^{0} \hat{G_{\sigma_{2}}}+D_{2}\left(i \omega+i\left(\vec{\xi}_{0} \cdot \vec{v}_{0}\right)-\kappa+\left(1-e^{-\frac{1}{\rho}}\right) \kappa \rho_{l}^{0} \hat{G_{\sigma_{2}}}\right)=0
\end{aligned}
$$




$$
M\left(\begin{array}{c}
A \\
B \\
C_{1} \\
C_{2} \\
D_{1} \\
D_{2}
\end{array}\right)=0
$$

The matrix $M$ is:

$$
M=\left(\begin{array}{lll}
M_{1} & M_{2} & M_{3}
\end{array}\right)
$$

where

$$
M_{1}=\left(\begin{array}{cc}
\omega+\vec{\xi}_{0} \cdot \vec{v}_{0} & 0 \\
0 & \omega+\vec{\xi}_{0} \cdot \vec{v}_{0} \\
\kappa\left(\hat{H}_{1}+c_{a} \hat{K_{1}}\right) & \kappa\left(\hat{H}_{1}+c_{a} \hat{K}_{1}\right) \\
\kappa\left(\hat{H}_{2}+c_{a} \hat{K_{2}}\right) & \kappa\left(\hat{H}_{2}+c_{a} \hat{K}_{2}\right) \\
\kappa\left(1-e^{-\frac{1}{\rho}}\right)\left(\hat{H}_{1}+c_{a} \hat{K}_{1}\right) & \kappa\left(1-e^{-\frac{1}{\rho}}\right)\left(\hat{H}_{1}+c_{a} \hat{K_{1}}\right) \\
\kappa\left(1-e^{-\frac{1}{\rho}}\right)\left(\hat{H}_{2}+c_{a} \hat{K_{2}}\right) & \kappa\left(1-e^{-\frac{1}{\rho}}\right)\left(\hat{H}_{2}+c_{a} \hat{K_{2}}\right)
\end{array}\right)_{6 \times 2}
$$




$$
M_{3}=\left(\begin{array}{cc}
0 & 0 \\
\rho_{l}^{0} \xi_{1} & \rho_{l}^{0} \xi_{2} \\
\kappa \rho_{l}^{0} \hat{G_{\sigma_{2}}} & 0 \\
0 & \kappa \rho_{l}^{0} \hat{G_{\sigma_{2}}} \\
i \omega+i\left(\overrightarrow{\xi_{0}} \cdot \vec{v}_{0}\right)-\kappa & 0 \\
+\left(1-e^{-\frac{1}{\rho}}\right) \kappa \rho_{l}^{0} \hat{G}_{\sigma_{2}} & i \omega+i\left(\overrightarrow{\xi_{0}} \cdot \overrightarrow{v_{0}}\right)-\kappa \\
0 & +\left(1-e^{-\frac{1}{\rho}}\right) \kappa \rho_{l}^{0} \hat{G_{2}}
\end{array}\right)_{6 \times 2}
$$

\subsubsection{Analysis}

Our goal is to analyze the dispersion coefficient $\omega(\xi)$. We want to determine if its imaginary part is positive or negative. A negative imaginary part would imply stability. A positive imaginary part would indicate that the perturbation grows, hence unstable. For example:

$$
e^{-i \omega t}=e^{-i(a+b i) t}=\underbrace{e^{-i a t} e^{b t}}_{\text {grows with time if } b>0}
$$

If we want the plane waves to be non-trivial, $A, B, C_{1}, C_{2}, D_{1}, D_{2}$ should not be all zero. So we are looking for non-trivial solutions to the system, i.e. solutions in the nullspace of this matrix $M$. We set the determinant of $M$ to be zero (non-zero determinant would imply no non-trivial solutions). The determinant of $M$ is:

$$
\begin{aligned}
& \operatorname{det}(M)= \\
& e^{-\frac{2}{\rho}}\left(\omega+\overrightarrow{\xi_{0}} \cdot \overrightarrow{v_{0}}\right)\left(\kappa-i\left(\omega+\overrightarrow{\xi_{0}} \cdot \overrightarrow{v_{0}}\right)\right)^{2}\left(-\hat{G_{\sigma_{2}}} \rho_{l}^{0} \kappa+e^{\frac{1}{\rho}}\left(\kappa\left(-1+\hat{G_{\sigma_{2}}}\right)+i\left(\omega+\overrightarrow{\xi_{0}} \cdot \overrightarrow{v_{0}}\right)\right)\right) \\
& \left(e ^ { \frac { 1 } { \rho } } \left(-\hat{H_{1}} \kappa \xi_{1}-\hat{H_{2}} \kappa \xi_{2}-c_{a} \kappa\left(\hat{K_{1}} \xi_{1}+\hat{K_{2}} \xi_{2}\right)-\kappa \xi_{1} v_{1}+\hat{G_{\sigma_{2}}} \kappa \xi_{1} v_{1}-\kappa \xi_{2} v_{2}+i \xi_{1}^{2} v_{1}^{2}\right.\right. \\
& \left.+\hat{G_{\sigma_{2}}} \kappa \xi_{2} v_{2}+2 i \xi_{1} \xi_{2} v_{1} v_{2}+i \xi_{2}^{2} v_{2}^{2}-\kappa \omega+\hat{G_{\sigma_{2}}} \kappa \omega+2 i \xi_{1} v_{1} \omega+2 i \xi_{2} v_{2} \omega+i \omega^{2}\right) \\
& \left.+\kappa \rho_{l}^{0}\left(\left(\hat{H}_{1}+c_{a} \hat{K_{1}}\right) \xi_{1}+\left(\hat{H}_{2}+c_{a} \hat{K_{2}}\right) \xi_{2}-\hat{G_{\sigma_{2}}} \xi_{1} v_{1}-\hat{G_{\sigma_{2}}} \xi_{2} v_{2}-\hat{G_{\sigma_{2}}} \omega\right)\right)
\end{aligned}
$$

At least one of these factors must be equal to zero.

Setting the first factor of (2.7) to be zero, then we could get:

$$
\omega=-\vec{\xi}_{0} \cdot \vec{v}_{0}
$$


The imaginary part of $\omega$ is equal to zero, so the disturbance neither decays nor grows.

Seting the second factor of (2.7) equal to zero,

$$
\omega=-\vec{\xi}_{0} \cdot \vec{v}_{0}-i \kappa
$$

the imaginary part is always less than zero, so it's always stable.

The third factor of (2.7) gives us:

$$
-\hat{G_{\sigma_{2}}} \rho_{l}^{0} \kappa+e^{\frac{1}{\rho}}\left(\kappa\left(-1+\hat{G_{\sigma_{2}}}\right)+i\left(\omega+\overrightarrow{\xi_{0}} \cdot \overrightarrow{v_{0}}\right)\right)=0
$$

which means:

$$
\left.\omega=-\vec{\xi}_{0} \cdot \vec{v}_{0}-i \kappa\left(1-\widehat{G_{\sigma_{2}}}+\widehat{G_{\sigma_{2}}} e^{-\frac{1}{\rho}} \rho_{l}^{0}\right)\right)
$$

Recall that $\widehat{G_{\sigma_{2}}}=e^{-\sigma_{2}^{2}|\vec{\xi}|^{2}}(2.4 \mathrm{~b})$, therefore

$$
0 \leq \widehat{G_{\sigma_{2}}} \leq 1
$$

When the imaginary part of $\omega$ is less than 0 , the swarm will be stable. i.e.

$$
-\left(1+\hat{G_{\sigma_{2}}}\left(-1+\rho_{l}^{0} e^{-\frac{1}{\rho}}\right)\right)<0
$$

which means

$$
\rho_{l}^{0}>e^{\frac{1}{\rho}}\left(1-\frac{1}{\hat{G_{\sigma_{2}}}}\right)
$$

With the help of (2.8), the right hand side of (2.9) is always less than 0 . We know that the density of the followers $\rho_{l}^{0}$ is always greater than zero. So the inequality (2.9) always holds. That means the system is always stable.

Seting the fourth term of (2.7) to be zero:

$$
\begin{aligned}
& e^{\frac{1}{\rho}}\left(-\hat{H_{1}} \kappa \xi_{1}-\hat{H_{2}} \kappa \xi_{2}-c_{a} \kappa\left(\hat{K_{1}} \xi_{1}+\hat{K_{2}} \xi_{2}\right)-\kappa \xi_{1} v_{1}+\hat{G_{\sigma_{2}}} \kappa \xi_{1} v_{1}-\kappa \xi_{2} v_{2}+i \xi_{1}^{2} v_{1}^{2}\right. \\
& \left.+\hat{G_{\sigma_{2}}} \kappa \xi_{2} v_{2}+2 i \xi_{1} \xi_{2} v_{1} v_{2}+i \xi_{2}^{2} v_{2}^{2}-\kappa \omega+\hat{G_{\sigma_{2}}} \kappa \omega+2 i \xi_{1} v_{1} \omega+2 i \xi_{2} v_{2} \omega+i \omega^{2}\right)+ \\
& \kappa \rho_{l}^{0}\left(\left(\hat{H}_{1}+c_{a} \hat{K_{1}}\right) \xi_{1}+\left(\hat{H_{2}}+c_{a} \hat{K_{2}}\right) \xi_{2}-\hat{G_{\sigma_{2}}} \xi_{1} v_{1}-\hat{G_{\sigma_{2}}} \xi_{2} v_{2}-\hat{G_{\sigma_{2}}} \omega\right)=0
\end{aligned}
$$


Using the quadratic formula, we could get the roots of (2.10):

$$
\begin{aligned}
& \omega=-\left(\vec{\xi}_{0} \cdot \vec{v}_{0}\right)-\frac{1}{2} i \kappa\left(1+\hat{G_{\sigma_{2}}}\left(-1+\rho_{l}^{0} e^{-\frac{1}{\rho}}\right)\right) \pm
\end{aligned}
$$

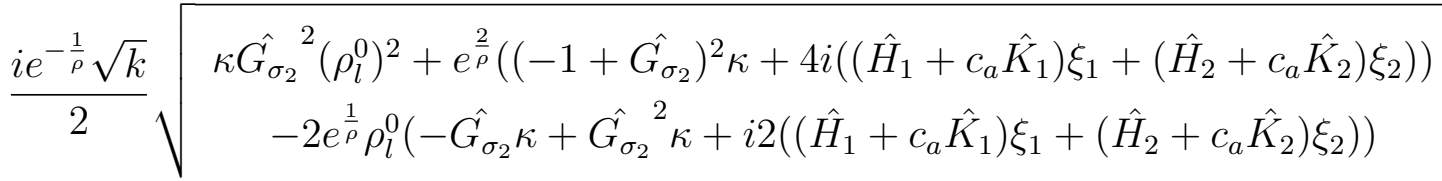

Substituting the values of $\hat{H}_{\sigma_{1}}, \hat{K_{\sigma_{3}}}$ and $\hat{G_{\sigma_{2}}}$ into $(2.11)$, then

$$
\begin{aligned}
& \left(\hat{H}_{1}+c_{a} \hat{K_{1}}\right) \xi_{1}+\left(\hat{H}_{2}+c_{a} \hat{K}_{2}\right) \xi_{2}=i\left|\vec{\xi}_{0}\right|^{2}\left[e^{-\sigma_{1}^{2}\left|\vec{\xi}_{0}\right|^{2}}+c_{a} \frac{1}{2}\left(-2+\sigma_{3}^{2}\left|\vec{\xi}_{0}\right|^{2}\right) e^{-\sigma_{3}^{2}\left|\vec{\xi}_{0}\right|^{2}}\right] \\
& \hat{G_{\sigma_{2}}}=e^{-\sigma_{2}^{2}\left|\vec{\xi}_{0}\right|^{2}}
\end{aligned}
$$

So

$$
\begin{aligned}
& \omega=-\left(\vec{\xi}_{0} \cdot \vec{v}_{0}\right)-\frac{1}{2} i \kappa\left(1+e^{-\sigma_{2}^{2}\left|\vec{\xi}_{0}\right|^{2}}\left(-1+e^{-\frac{1}{\rho}} \rho_{l}^{0}\right)\right) \pm
\end{aligned}
$$

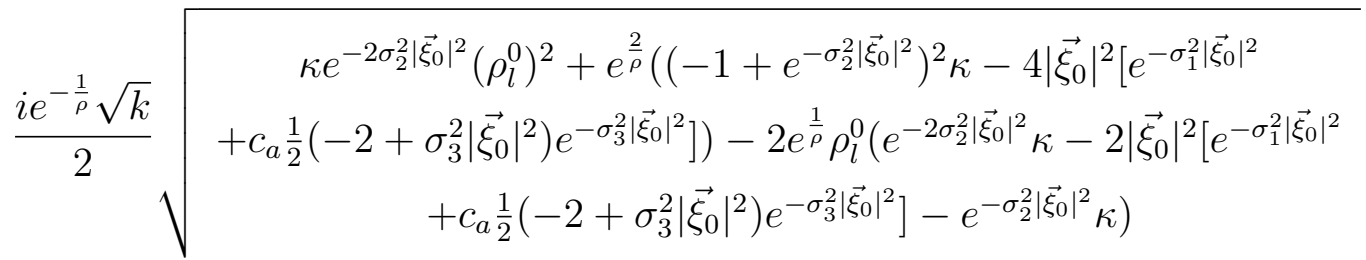

If the imaginary part of $\omega$ is positive then the system will be unstable. If the value under the square root is negative, then the imaginary part of $\omega$ is negative. So here we just consider the value under the square root is positive. When the follow inequality holds, the system will be unstable.

$$
\begin{aligned}
& -\frac{1}{2} \kappa\left(1+e^{-\sigma_{2}^{2}\left|\vec{\xi}_{0}\right|^{2}}\left(-1+e^{-\frac{1}{\rho}} \rho_{l}^{0}\right)\right) \pm
\end{aligned}
$$

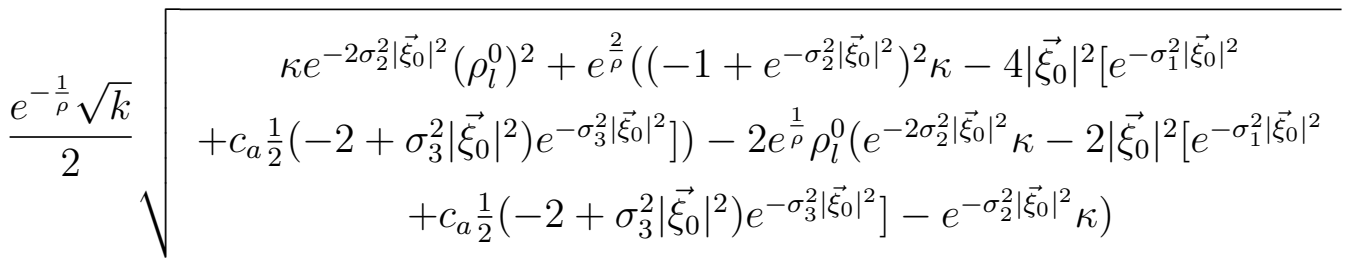

$$
\begin{aligned}
& >0
\end{aligned}
$$


For the '-' choice of ' \pm ' in (2.12), the left hand side of (2.12) is always less than 0, which means it is always stable. So we explore the alternative '+' choice,

$$
\begin{aligned}
& -\frac{1}{2} \kappa\left(1+e^{-\sigma_{2}^{2}\left|\vec{\xi}_{0}\right|^{2}}\left(-1+e^{-\frac{1}{\rho}} \rho_{l}\right)\right)+ \\
& \begin{array}{l}
\frac{e^{-\frac{1}{\rho}} \sqrt{k}}{2} \sqrt{\kappa e^{-2 \sigma_{2}^{2}\left|\vec{\xi}_{0}\right|^{2}}\left(\rho_{l}^{0}\right)^{2}+e^{\frac{2}{\rho}}\left(\left(-1+e^{-\sigma_{2}^{2}\left|\vec{\xi}_{0}\right|^{2}}\right)^{2} \kappa-4\left|\vec{\xi}_{0}\right|^{2}\left[e^{-\sigma_{1}^{2}\left|\vec{\xi}_{0}\right|^{2}}\right.\right.} \\
\left.\left.+c_{a} \frac{1}{2}\left(-2+\sigma_{3}^{2}\left|\vec{\xi}_{0}\right|^{2}\right) e^{-\sigma_{3}^{2}\left|\vec{\xi}_{0}\right|^{2}}\right]\right)-2 e^{\frac{1}{\rho}} \rho_{l}^{0}\left(e^{-2 \sigma_{2}^{2}\left|\vec{\xi}_{0}\right|^{2}} \kappa-2\left|\vec{\xi}_{0}\right|^{2}\left[e^{-\sigma_{1}^{2}\left|\vec{\xi}_{0}\right|^{2}}\right.\right. \\
\left.+c_{a} \frac{1}{2}\left(-2+\sigma_{3}^{2}\left|\vec{\xi}_{0}\right|^{2}\right) e^{\left.-\sigma_{3}^{2}\left|\vec{\xi}_{0}\right|^{2}\right]}-e^{-\sigma_{2}^{2}\left|\vec{\xi}_{0}\right|^{2}} \kappa\right)
\end{array} \\
& >0
\end{aligned}
$$

Simplifying the inequality, we get

$$
4 \kappa e^{\frac{1}{\rho}}\left|\vec{\xi}_{0}\right|^{2}\left[e^{-\sigma_{1}^{2}\left|\vec{\xi}_{0}\right|^{2}}+c_{a} \frac{1}{2}\left(-2+\sigma_{3}^{2}\left|\vec{\xi}_{0}\right|^{2}\right) e^{-\sigma_{3}^{2}\left|\vec{\xi}_{0}\right|^{2}}\right]\left(e^{\frac{1}{\rho}}-\rho_{l}^{0}\right)<0
$$

Since $e^{\frac{1}{\rho}}>1, e^{\frac{1}{\rho}}-\rho_{l}^{0}>0$. The only concern in $(2.13)$ is:

$$
e^{-\sigma_{1}^{2}\left|\vec{\xi}_{0}\right|^{2}}+c_{a} \frac{1}{2}\left(-2+\sigma_{3}^{2}\left|\vec{\xi}_{0}\right|^{2}\right) e^{-\sigma_{3}^{2}\left|\vec{\xi}_{0}\right|^{2}}<0
$$

This is the same inequality as for the leaderless swarm model mentioned in [20]. The results in [20] tell us that disturbances to the uniform state will die out if $0<c_{a} \leq 1$, and if $c_{a}>1$, infinite swarms are unstable to small wavenumber disturbances.

\subsubsection{Conclusion}

Our analysis shows that the stability properties are the same as for leaderless swarms. The stability properties depend on the relative strength of the attraction compared with the repulsion and the orientation. If $0<c_{a} \leq 1$, a plane wave perturbation with any wavenumber will not grow. If $c_{a}>1$, plane wave perturbation with small wave numbers will grow exponentially. Conversely, plane wave perturbation with large wave numbers will damp out.

\subsection{Verification}

We would like to verify our analysis result through simulation. We discretize the continuous model into $N$ individuals interacting with one another. The discretized 
method which systematically transforms convolutions into summations can be found in [20]. The resulting discretized desired velocity for each individual can be represented by the following equations:

$$
\begin{aligned}
\vec{v}_{f d, i}= & \sum_{j=1}^{N} H_{\sigma_{1}}\left(\vec{x}_{i}-\vec{x}_{j}\right)+\frac{\sum_{j=1}^{N} G_{\sigma_{2}}\left(\vec{x}_{i}-\vec{x}_{j}\right) \vec{v}\left(\vec{x}_{j}\right)}{\sum_{j=1}^{N} G_{\sigma_{2}}\left(\vec{x}_{i}-\vec{x}_{j}\right)}+c_{a} \sum_{j=1}^{N} K_{\sigma_{3}}\left(\vec{x}_{i}-\vec{x}_{j}\right) \\
\vec{v}_{l d, i}= & \left(1-\exp \left(-\frac{\sum_{j=1}^{N} G_{\sigma_{2}}\left(\vec{x}_{i}-\vec{x}_{j}\right)}{\rho}\right)\right)\left(\sum_{j=1}^{N} H_{\sigma_{1}}\left(\vec{x}_{i}-\vec{x}_{j}\right)+\frac{\sum_{j=1}^{N} G_{\sigma_{2}}\left(\vec{x}_{i}-\vec{x}_{j}\right) \vec{v}\left(\vec{x}_{j}\right)}{\sum_{j=1}^{N} G_{\sigma_{2}}\left(\vec{x}_{i}-\vec{x}_{j}\right)}\right. \\
& \left.+c_{a} \sum_{j=1}^{N} K_{\sigma_{3}}\left(\vec{x}_{i}-\vec{x}_{j}\right)\right)+\exp \left(-\frac{\sum_{j=1}^{N} G_{\sigma_{2}}\left(\vec{x}_{i}-\vec{x}_{j}\right)}{\rho}\right) \vec{g}_{i}
\end{aligned}
$$

Once the desired velocity has been computed, the velocity for each individual could be updated.

All calculations were performed using both ideal interactions and the QualNet simulator with realistic wireless communication, and results from both were found to be in reasonable agreement, see Figs 2.1(c) and 2.2. The value of the parameters we used could be found in Table 2.1.

Table 2.1: Parameter values

\begin{tabular}{|c|c|}
\hline \hline$\sigma_{1}$ & $3 / 4$ \\
\hline$\sigma_{2}$ & $9 / 4$ \\
\hline$\sigma_{3}$ & $15 / 4$ \\
\hline$\rho$ & $24 / 81 \pi$ \\
\hline$c_{a}$ & 10 \\
\hline$\kappa$ & 1 \\
\hline \hline
\end{tabular}

To validate our analysis, we performed a series of computational experiments. We choose $c_{a}=10$ in which case a constant density swarm will be unstable. Without leaders, an initially disordered swarm will collapse into an axisymmetric attractor with non-constant density moving in a single direction [21]. The final direction of propagation depends upon the initial conditions. One can control the direction of motion by adding some covert leaders which have a preference for a specific direction $\vec{g}$. One 


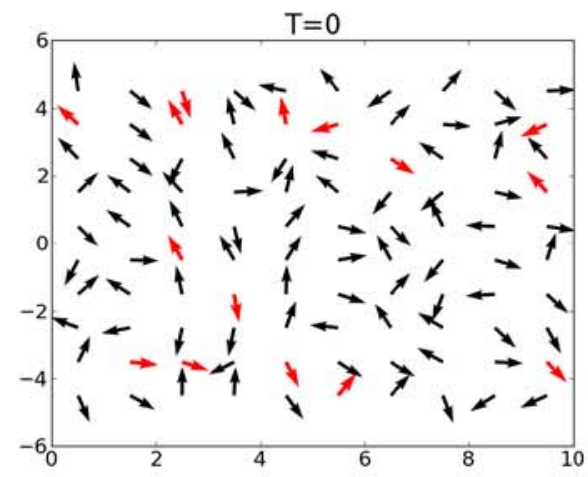

(a) Initial condition

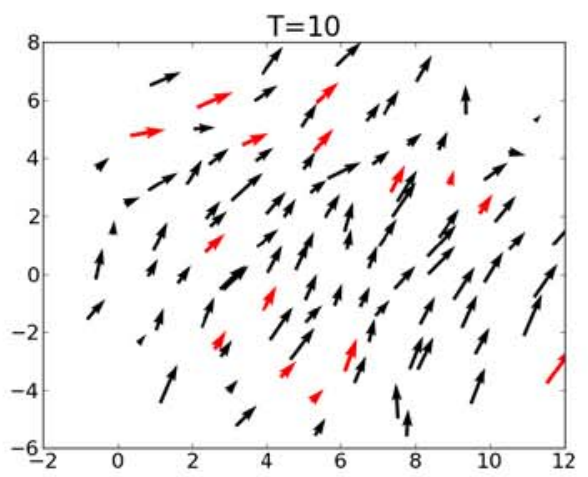

(b) Rapid reorganization

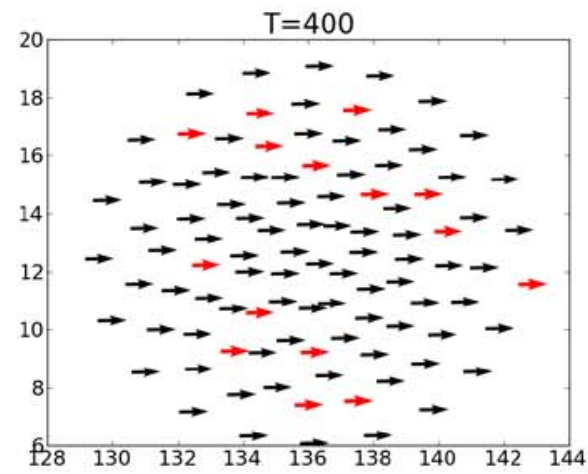

(c) Final orientation in the desired direction.

Figure 2.1: In this swarm, $15 \%$ of the individuals are covert leaders using the nonlinear model. The initial velocity is random with the speed varying from 0 to 1 . The leaders possess additional information $\vec{g}=[1,0]^{T}$. Red arrows represent the leaders, and black arrows represent the followers..

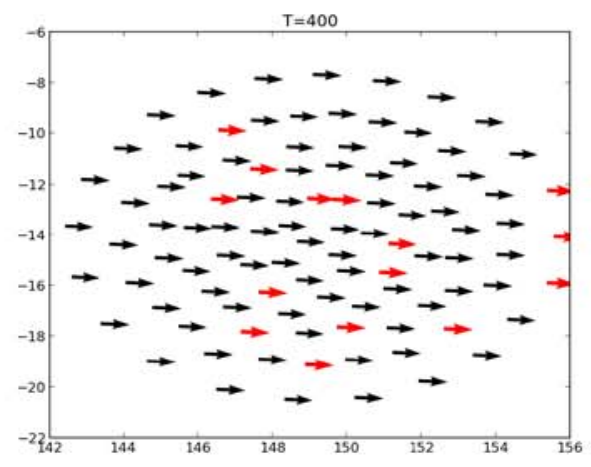

Figure 2.2: Final swarm configuration using the QualNet simulator 
example of this collapse is shown in Figure 2.1. In Figure 2.1c we observe that the leaders appear to be distributed throughout the swarm, especially near the center. In contrast, in the linear model, the leaders will accumulate into the front. In detail, in the linear model, all leaders act on additional information in the same way. Specifically, this model suggested by Couzin et. al. has the form [10],

$$
\vec{v}_{l d}=(1-\alpha)\left(H_{\sigma_{1}} *\left(\rho_{f}+\rho_{l}\right)+\frac{G_{\sigma_{2}} *\left(\rho_{f} \vec{v}_{f}+\rho_{l} \vec{v}_{l}\right)}{G_{\sigma_{2}} *\left(\rho_{f}+\rho_{l}\right)}+c_{a} K_{\sigma_{3}} *\left(\rho_{f}+\rho_{l}\right)\right)+\alpha \vec{g}
$$

in contrast to $(1.4 \mathrm{~d})$. The weight $\alpha$ is often taken to be 0.5 . The discretized form is

$$
\begin{aligned}
\vec{v}_{l d, i}= & (1-\alpha)\left[\sum_{j=1}^{N} H_{\sigma_{1}}\left(\vec{x}_{i}-\vec{x}_{j}\right)+\frac{\sum_{j=1}^{N} G_{\sigma_{2}}\left(\vec{x}_{i}-\vec{x}_{j}\right) \vec{v}\left(\vec{x}_{j}\right)}{\sum_{j=1}^{N} G_{\sigma_{2}}\left(\vec{x}_{i}-\vec{x}_{j}\right)}+c_{a} \sum_{j=1}^{N} K_{\sigma_{3}}\left(\vec{x}_{i}-\vec{x}_{j}\right)\right] \\
& +\alpha \vec{g}_{i}
\end{aligned}
$$

in contrast to $(2.14 \mathrm{~b})$. The difference is evident when comparing the results in Figure 2.1c with those in Figure 2.3.

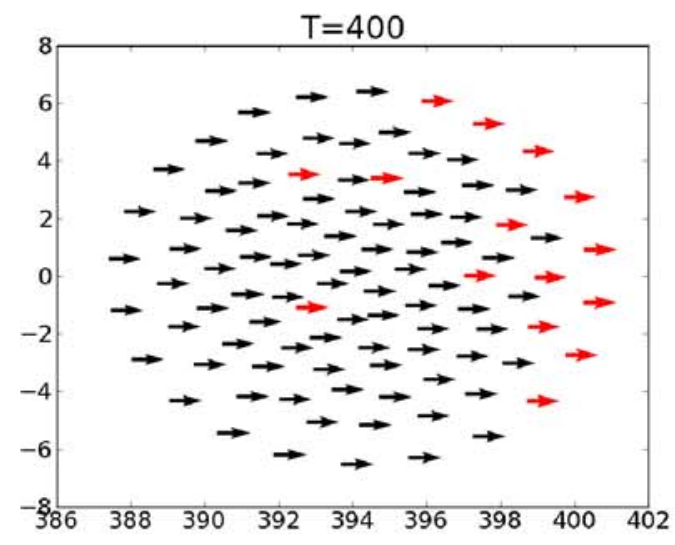

Figure 2.3: In this swarm, all parameters are the same as in Figure 3 except we use the linear leadership model. Notice that most of the leaders aggregate at the leading edge.

We have also conducted simulations using the QualNet network simulator. Due to the broadcast nature of wireless communications, uncoordinated communications among nodes within a dense swarm would result in extensive packet collisions and hence information loss. To address this issue, we have implemented a TDMA-like 
transmission scheme, where the notion of time is divided into periods of "steps." Each step is further divided into multiple constant time slots, which are assigned to nodes within a swarm. Therefore, nodes are only allowed to broadcast their position and velocity information to their neighbors on the assigned time slots. At the end of each step, nodes update their velocities based on information received during the step. The QualNet simulations use the default IEEE 802.11b Physical Layer protocol parameters for wireless communications and the Two-Ray path-loss model without fading.

\subsection{Conclusion}

Our analysis shows that the stability properties of our model are the same as for leaderless swarms, and it is consistent with our simulation results both with ideal communication and simulations with realistic wireless protocols using QualNet. This indicates that it is possible to inject additional information into a swarm without altering its dynamical properties including the swarm's stable configurations. Furthermore, the nonlinear leadership model allows leaders to embed themselves in the swarm rather than aggregating in the front. 


\section{Chapter 3 COLLECTIVE DECISIONS}

Informed individuals within a group may differ in their preferred direction due to differences in experience or motivation. Groups of animals often have to make collective decisions. They may need to move together to a specific resource, for example, a nest site or food source [10]. We consider a scenario where there are two groups of leaders possessing different external information, say $\vec{g}_{1}, \vec{g}_{2}$. For simplicity, we assume that there are equal numbers of each type of leader in the swarm, and that $\vec{g}_{2}$, is the reflection of $\vec{g}_{1}$ about the x-axis. In other words, $\arg \left(\vec{g}_{1}+\vec{g}_{2}\right)=0$. We define the information differential, a measurement of the difference between the two groups of leaders, to be

$$
\Delta \theta=\arg \left(\vec{g}_{1}-\vec{g}_{2}\right) .
$$

We explore whether swarms arrive at consensus or splinter, even though informed individuals do not explicitly know whether there are any other informed individuals.

\section{$3.1 \quad$ Experiments}

To understand the motion of the group, we define the average velocity of the group to be

$$
\vec{v}=\frac{1}{N} \sum_{j=1}^{N} \vec{v}_{j},
$$

where $N$ is the total number of individuals and $\vec{v}_{i}$ is the velocity of the $i$ th individual. We can define the direction of the group to be $\theta=\arg (\vec{v})$. In our experiments, we vary the information differential $\Delta \theta$ from 0 to $\pi$. Other parameter values such as zone size are as listed in Table 2.1. For each value $\Delta \theta$ between 0 and $\pi$ in 10 even increments, we conducted 50 experiments and measured the group direction after the 
swarm stabilized. In each experiment, the initial velocity are randomly chosen. 0.07 of the individuals are leaders with one type of information $\left(\vec{g}_{1}\right)$ and an equal number have the other type of information $\left(\vec{g}_{2}\right)$. As seen in Figure 3.1, when $\Delta \theta$ is small, the swarm arrives at a consensus and a compromise is achieved. However, there is a bifurcation

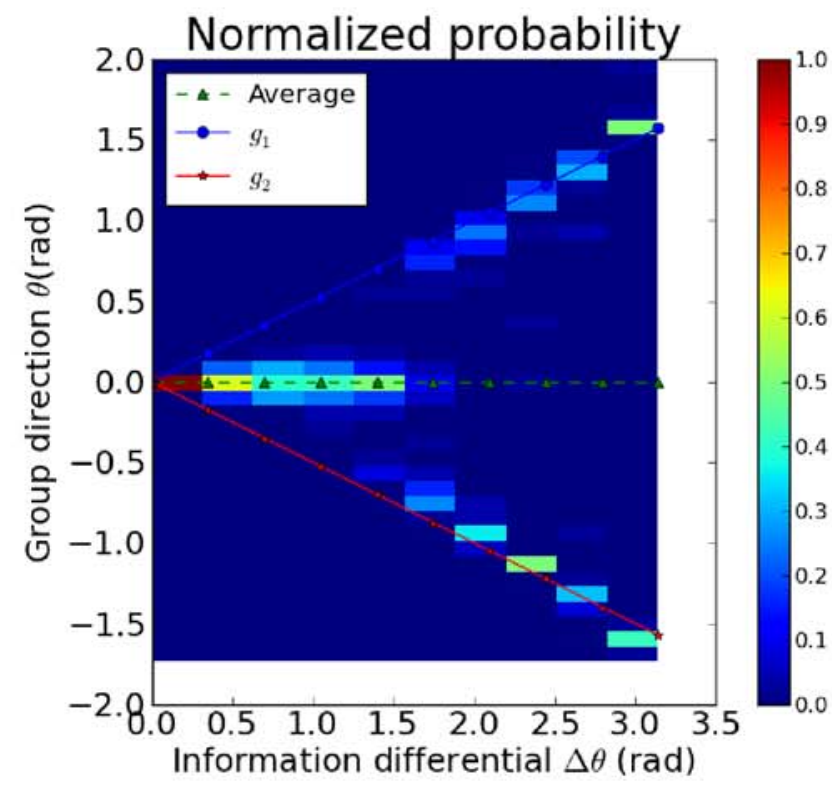

Figure 3.1: Normalized probability of the group direction in an experiment with two groups of leaders. In this case, $7 \%$ of the individuals in the swarm have one type of additional information, and another $7 \%$ have the other type. The $\mathrm{x}$-axis represents difference between the additional information of the two types of leaders. The $y$-axis represents the direction of the whole group. The color represents the likelihood of the outcome shown on the graph.

roughly at $\pi / 2$. Beyond this point, the swarm will follow one group of leaders while a small portion of individuals will splinter away from the swarm (Figs 3.2), so that the average swarm direction is aligned with one group of leaders. The precise position of the bifurcation is sensitive to $\rho$, the nonlinearity in the leadership model $(1.4 \mathrm{~d})$. The results could be found in Figure 3.3. The bifurcation will shift left with increasing $\rho$. That means with a larger $\rho$, a smaller information differential will cause the swarm to split. The bifurcation will shift right with decreasing $\rho$. That means with a smaller $\rho$, the swarm will choose the average direction even with a larger information differential. 

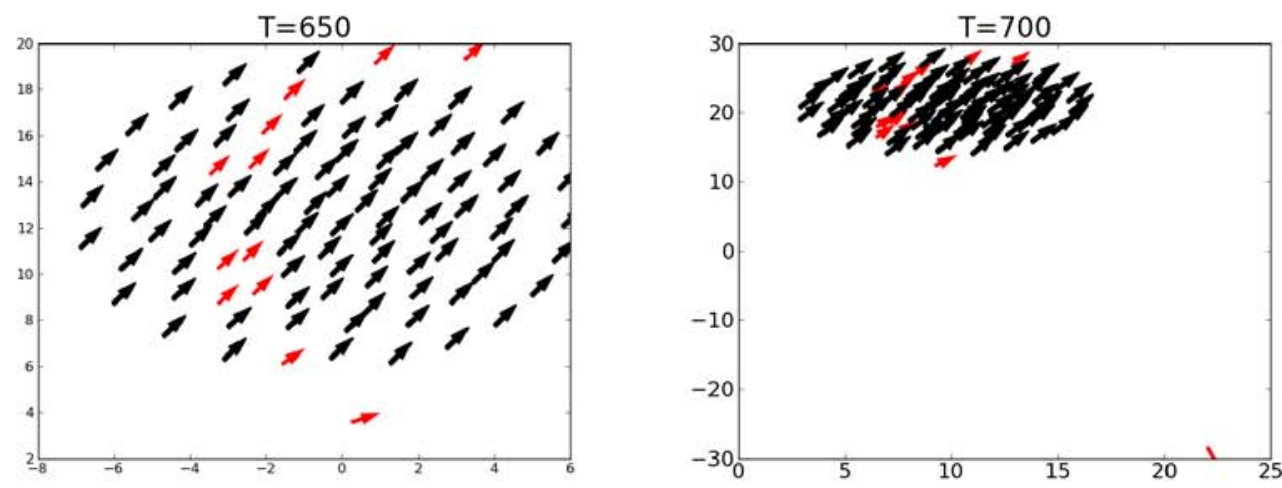

Figure 3.2: Leaders from one group carrying away the swarm and most of the other leaders. However some leaders splinter away from the swarm.

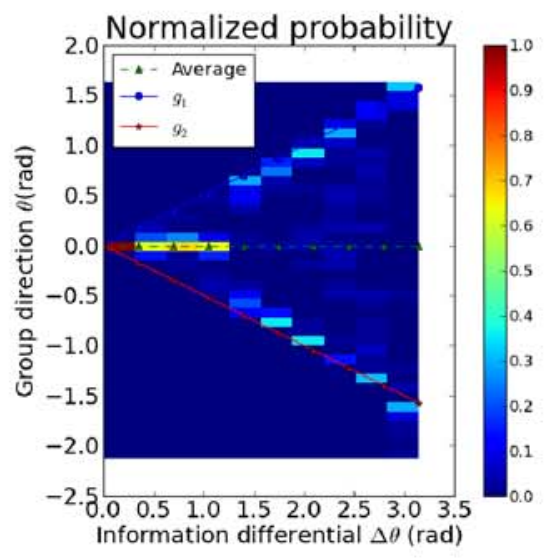

(a) Shifting left: $\rho=0.102$.

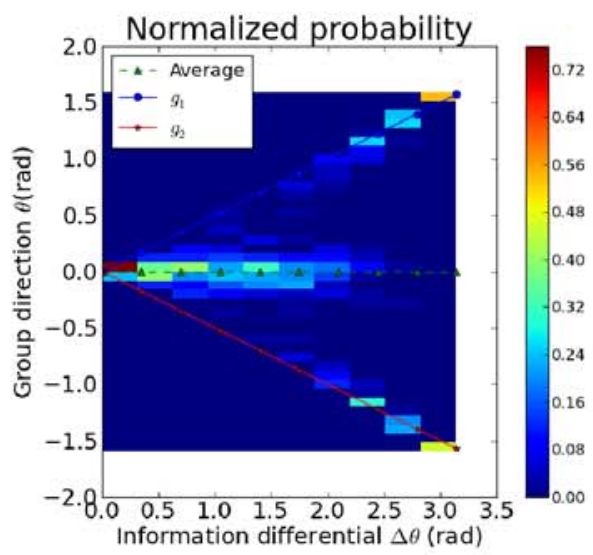

(b) Shifting right: $\rho=0.088$.

Figure 3.3: The position of the bifurcation point depends on $\rho$.

To quantify the splintering events, we define the splinter fraction to be $l / N$ where $l$ is the number of individuals who have traveled beyond a cutoff distance $c$ from the swarm centroid. We choose $c$ to be 500, a value large enough to remove any ambiguity about whether an individual has left the swarm or not. Measurements of the splinter fraction from the experiment in Figure 3.1 are shown in Figure 3.4.

To understand if there is a dynamic instability in the swarm, we repeat the experiment with initial conditions that are symmetric about the $\mathrm{x}$ axis. If the swarm is dynamically unstable, errors from roundoff will be amplified and the system will yield similar results to experiments with completely random initial conditions. We observe 


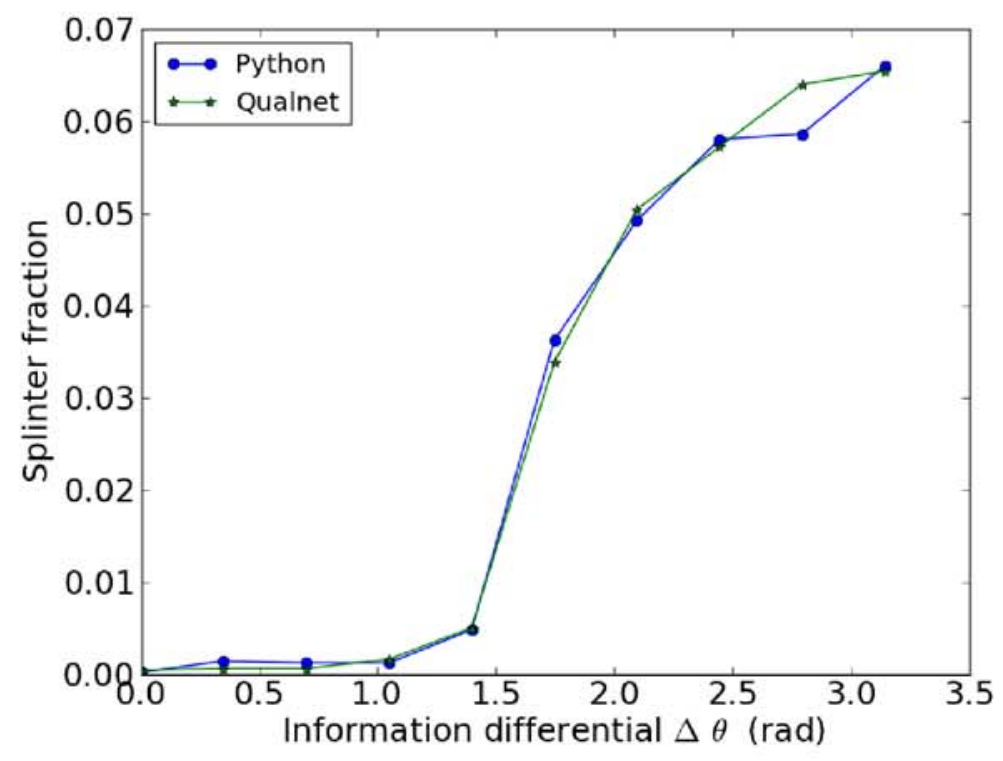

Figure 3.4: Measurements of the splinter fraction from the experiments described in Figure 3.1. The simulations using ideal interactions and the QualNet simulations are in excellent agreement.

that this does not happen. The swarm reorganizes, and both groups of leaders symmetrically split from the swarm as shown in Figure 3.5. We conclude that splintering
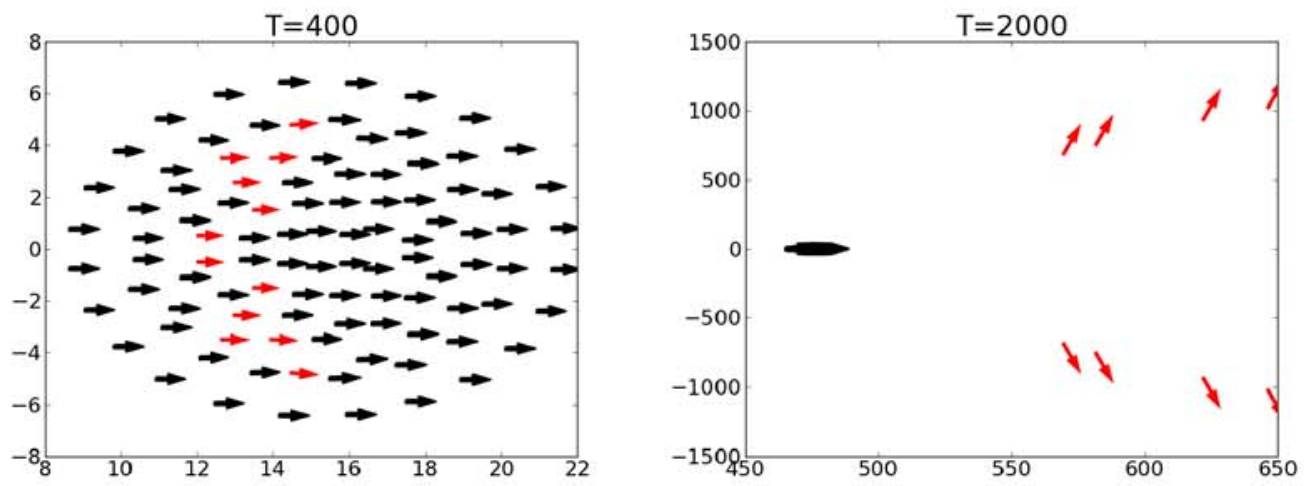

(a) Initial reorganization with symmetric (b) Stable symmetric splintering of leaders groups of leaders.

Figure 3.5: With symmetric initial data, the swarm remains stable even though the symmetry is not enforced as the swarm evolves.

is a dynamically stable process that is continuously dependent on initial conditions. 
If we perform an experiment with disjoint groups of leaders as shown in Figure 3.6, we see that the bifurcation occurs at roughly the same position. Once again, the QualNet simulations show excellent agreement with simulations using ideal communications.

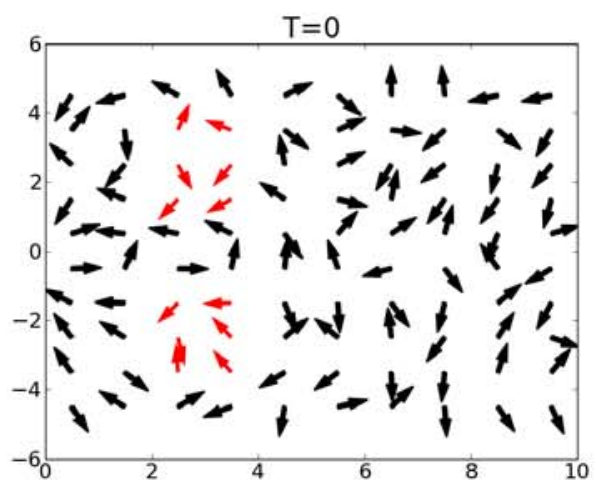

Figure 3.6: Initial condition for the experiments with disjoint leaders
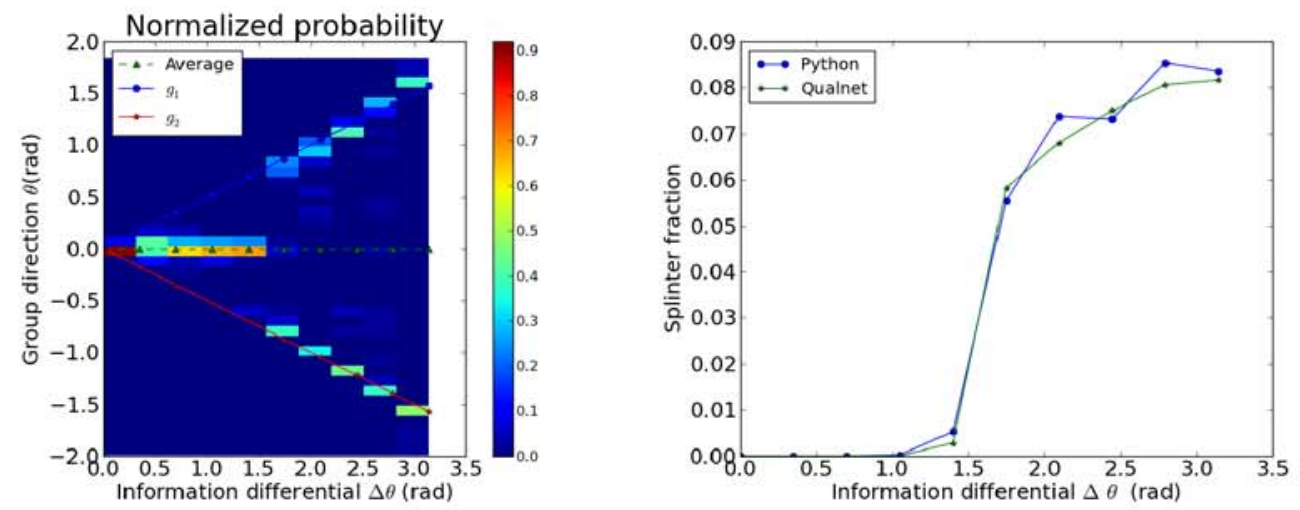

Figure 3.7: Random initial velocities but with symmetry imposed along the $\mathrm{x}$ axis.

We performed one final experiment to understand the bifurcation by removing all random disturbances from the initial profile. In this case, all individuals are initialized on a regular lattice with velocity vector $[1,0]^{T}$. We find that the bifurcation is sharp as shown in Figure 3.8 .

To conclude, we find that when the information differential is small, the swarm will arrive at a consensus and follow the average desired direction. When the information differential is great, one group of leaders will splinter from the rest of the swarm. 


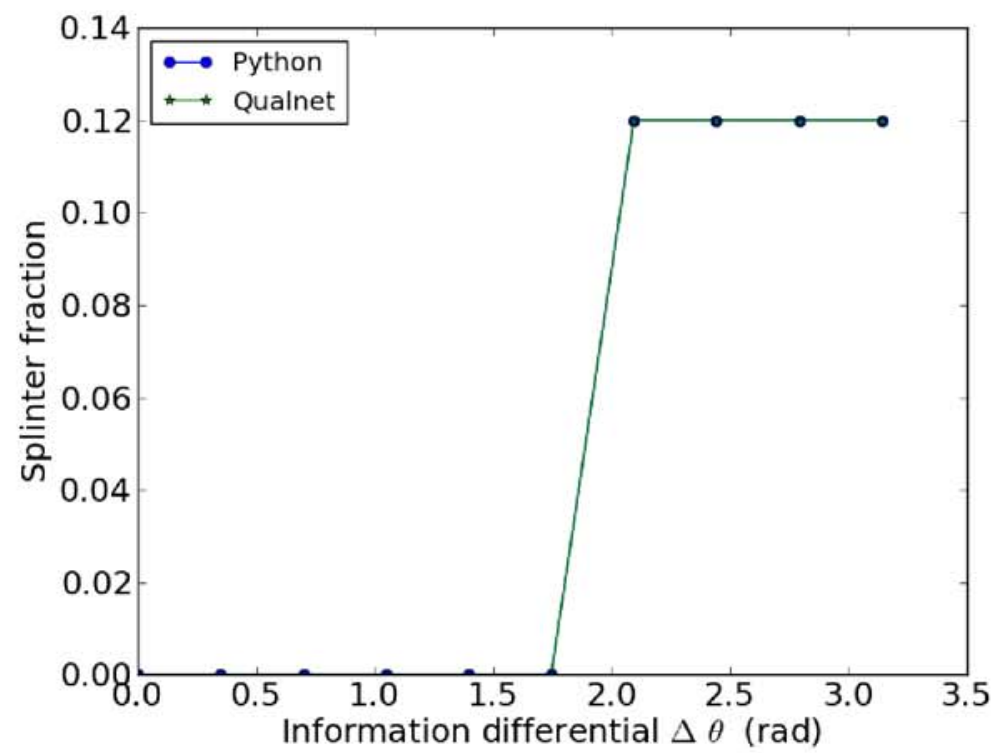

Figure 3.8: Splinter fraction from initial conditions on a regular lattice with initial velocity vector $[1,0]^{T}$.

All results were validated using simulations with ideal communication and with realistic wireless protocols using QualNet.

\subsection{Mathematical Analysis for the bifurcation}

From the experiment above, we find that for a specific $\rho$, the bifurcation point is around $\pi / 2$. However, we would like to know the bifurcation point mathematically, so that we could predict when the bifurcation will happen. Here we perform two case studies with just two individuals. In the first case, both of these two individuals are leaders. In the second case, each individual has a big mass so that each individual represents the center of mass of a large sub-swarm.

\subsubsection{Case study I: Two individuals}

Suppose that there are only two individuals in a swarm. Both are leaders, containing different types of external information, $\vec{g}_{1}$ and $\vec{g}_{2}$ (Figure 3.9). We want to find the relative positions and the velocities for both when they are in a stationary configuration. If there is a stationary configuration, then the acceleration should be 


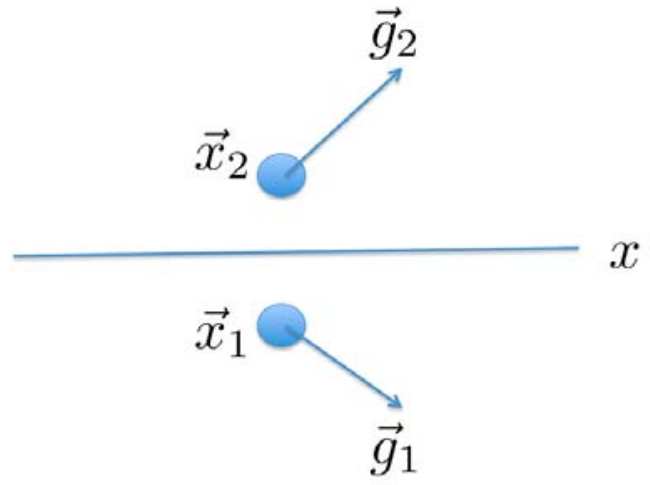

Figure 3.9: two individuals with different type of external information

equal to 0 , i.e the desired velocity is equal to the current velocity. From (1.4d), we know that

$$
\begin{aligned}
\vec{v}_{l d}= & \left(1-e^{-\frac{G_{\sigma_{2}} *\left(\rho_{f}+\rho_{l}\right)}{\rho}}\right)\left(H_{\sigma_{1}} *\left(\rho_{f}+\rho_{l}\right)+\frac{G_{\sigma_{2}} *\left(\rho_{f} \vec{v}_{f}+\rho_{l} \vec{v}_{l}\right)}{G_{\sigma_{2}} *\left(\rho_{f}+\rho_{l}\right)}\right. \\
& \left.+c_{a} K_{\sigma_{3}} *\left(\rho_{f}+\rho_{l}\right)\right)+e^{-\frac{G_{\sigma_{2}} *\left(\rho_{f}+\rho_{l}\right)}{\rho}} \vec{g}
\end{aligned}
$$

So for leader 1 , which is below the x-axis, containing external information $\vec{g}_{1}$, the velocity could be expressed as follows:

$$
\begin{aligned}
\vec{v}_{l d 1}= & \left(1-e^{-\frac{G_{\sigma_{2}} *\left(\rho_{f}+\rho_{l}\right)}{\rho}}\right)\left(H_{\sigma_{1}} *\left(\rho_{f}+\rho_{l}\right)+\frac{G_{\sigma_{2}} *\left(\rho_{f} \vec{v}_{f}+\rho_{l} \vec{v}_{l}\right)}{G_{\sigma_{2}} *\left(\rho_{f}+\rho_{l}\right)}\right. \\
& \left.+c_{a} K_{\sigma_{3}} *\left(\rho_{f}+\rho_{l}\right)\right)+e^{-\frac{G_{\sigma_{2}} *\left(\rho_{f}+\rho_{l}\right)}{\rho}} \vec{g}_{1}
\end{aligned}
$$

Suppose the velocity for both individuals at steady state is $\vec{v}_{0}$, then

$$
\vec{v}_{l d 1}=\vec{v}_{f}=\vec{v}_{l}=\vec{v}_{0}
$$


Plug it into (3.3), then we get:

$$
\begin{aligned}
& \vec{v}_{0}=\left(1-e^{-\frac{G_{\sigma_{2}} *\left(\rho_{f}+\rho_{l}\right)}{\rho}}\right)\left(H_{\sigma_{1}} *\left(\rho_{f}+\rho_{l}\right)+\frac{G_{\sigma_{2}} *\left(\rho_{f} \vec{v}_{0}+\rho_{l} \vec{v}_{0}\right)}{G_{\sigma_{2}} *\left(\rho_{f}+\rho_{l}\right)}+c_{a} K_{\sigma_{3}} *\left(\rho_{f}+\rho_{l}\right)\right) \\
& +e^{-\frac{G_{\sigma_{2} *\left(\rho_{f}+\rho_{l}\right)}}{\rho}} \vec{g}_{1} \\
& =\left(1-e^{-\frac{G_{\sigma_{2}} *\left(\rho_{f}+\rho_{l}\right)}{\rho}}\right)\left(H_{\sigma_{1}} *\left(\rho_{f}+\rho_{l}\right)+\vec{v}_{0}+c_{a} K_{\sigma_{3}} *\left(\rho_{f}+\rho_{l}\right)\right)+e^{-\frac{G_{\sigma_{2}} *\left(\rho_{f}+\rho_{l}\right)}{\rho}} \vec{g}_{1} \\
& =\left(1-e^{-\frac{G_{\sigma_{2}} *\left(\rho_{f}+\rho_{l}\right)}{\rho}}\right)\left(H_{\sigma_{1}} *\left(\rho_{f}+\rho_{l}\right)+c_{a} K_{\sigma_{3}} *\left(\rho_{f}+\rho_{l}\right)\right)+e^{-\frac{G_{\sigma_{2}} *\left(\rho_{f}+\rho_{l}\right)}{\rho}} \vec{g}_{1} \\
& +\left(1-e^{-\frac{G \sigma_{2} *\left(\rho_{f}+\rho_{l}\right)}{\rho}}\right) \vec{v}_{0} \\
& \Rightarrow\left(1-1+e^{-\frac{G_{\sigma_{2}} *\left(\rho_{f}+\rho_{l}\right)}{\rho}}\right) \vec{v}_{0}=\left(1-e^{-\frac{G_{\sigma_{2}} *\left(\rho_{f}+\rho_{l}\right)}{\rho}}\right)\left(H_{\sigma_{1}} *\left(\rho_{f}+\rho_{l}\right)+c_{a} K_{\sigma_{3}} *\left(\rho_{f}+\rho_{l}\right)\right) \\
& +e^{-\frac{G_{\sigma_{2}} *\left(\rho_{f}+\rho_{l}\right)}{\rho}} \vec{g}_{1} \\
& \Rightarrow \quad e^{-\frac{G_{\sigma_{2}} *\left(\rho_{f}+\rho_{l}\right)}{\rho}}\left(\vec{v}_{0}-\vec{g}_{1}\right)=\left(1-e^{-\frac{G_{\sigma_{2}} *\left(\rho_{f}+\rho_{l}\right)}{\rho}}\right)\left(H_{\sigma_{1}} *\left(\rho_{f}+\rho_{l}\right)+c_{a} K_{\sigma_{3}} *\left(\rho_{f}+\rho_{l}\right)\right) \\
& \Rightarrow \quad \vec{v}_{0}-\vec{g}_{1}=\left(e^{\frac{G_{\sigma_{2}} *\left(\rho_{f}+\rho_{l}\right)}{\rho}}-1\right)\left(H_{\sigma_{1}} *\left(\rho_{f}+\rho_{l}\right)+c_{a} K_{\sigma_{3}} *\left(\rho_{f}+\rho_{l}\right)\right)
\end{aligned}
$$

Finally we get:

$$
\vec{g}_{1}=\vec{v}_{0}-\left(e^{\frac{G_{\sigma_{2}} *\left(\rho_{f}+\rho_{l}\right)}{\rho}}-1\right)\left(H_{\sigma_{1}} *\left(\rho_{f}+\rho_{l}\right)+c_{a} K_{\sigma_{3}} *\left(\rho_{f}+\rho_{l}\right)\right)
$$

Similarly, for leader 2 , which is above the x-axis, containing external information $\vec{g}_{2}$, we get:

$$
\vec{g}_{2}=\vec{v}_{0}-\left(e^{\frac{G_{\sigma_{2}} *\left(\rho_{f}+\rho_{l}\right)}{\rho}}-1\right)\left(H_{\sigma_{1}} *\left(\rho_{f}+\rho_{l}\right)+c_{a} K_{\sigma_{3}} *\left(\rho_{f}+\rho_{l}\right)\right)
$$

The right hand side of (3.4) and (3.5) are exactly the same, but we need to note that the leaders' positions are different, i.e in the convolution or the integration

$$
\begin{aligned}
& \int H(x-y) \rho(y) d y \\
& \int K(x-y) \rho(y) d y
\end{aligned}
$$


$x$ are different. So the equations (3.4) and (3.5) could hold together. The discretized form for the leaders' desired velocity could be represented as:

$$
\begin{aligned}
\vec{v}_{l d, i}= & \left(1-\exp \left(-\frac{\sum_{j=1}^{N} G_{\sigma_{2}}\left(\vec{x}_{i}-\vec{x}_{j}\right)}{\rho}\right)\right)\left(\sum_{j=1}^{N} H_{\sigma_{1}}\left(\vec{x}_{i}-\vec{x}_{j}\right)+\frac{\sum_{j=1}^{N} G_{\sigma_{2}}\left(\vec{x}_{i}-\vec{x}_{j}\right) \vec{v}\left(\vec{x}_{j}\right)}{\sum_{j=1}^{N} G_{\sigma_{2}}\left(\vec{x}_{i}-\vec{x}_{j}\right)}\right. \\
& \left.+c_{a} \sum_{j=1}^{N} K_{\sigma_{3}}\left(\vec{x}_{i}-\vec{x}_{j}\right)\right)+\exp \left(-\frac{\sum_{j=1}^{N} G_{\sigma_{2}}\left(\vec{x}_{i}-\vec{x}_{j}\right)}{\rho}\right) \vec{g}_{i}
\end{aligned}
$$

If there is a stationary configuration, then for leader 1 , i.e $i=1, N=2$ :

$$
\begin{aligned}
& \vec{v}_{0}=\left(1-\exp \left(-\frac{\sum_{j=1}^{N} G_{\sigma_{2}}\left(\vec{x}_{i}-\vec{x}_{j}\right)}{\rho}\right)\right)\left(\sum_{j=1}^{N} H_{\sigma_{1}}\left(\vec{x}_{i}-\vec{x}_{j}\right)\right. \\
& \left.\vec{v}_{0}+c_{a} \sum_{j=1}^{N} K_{\sigma_{3}}\left(\vec{x}_{i}-\vec{x}_{j}\right)\right)+\exp \left(-\frac{\sum_{j=1}^{N} G_{\sigma_{2}}\left(\vec{x}_{i}-\vec{x}_{j}\right)}{\rho}\right) \vec{g}_{1} \\
& =\left(1-\exp \left(-\frac{\sum_{j=1}^{N} G_{\sigma_{2}}\left(\vec{x}_{i}-\vec{x}_{j}\right)}{\rho}\right)\right)\left(\sum_{j=1}^{N} H_{\sigma_{1}}\left(\vec{x}_{i}-\vec{x}_{j}\right)+c_{a} \sum_{j=1}^{N} K_{\sigma_{3}}\left(\vec{x}_{i}-\vec{x}_{j}\right)\right) \\
& +\left(1-\exp \left(-\frac{G_{\sigma_{2}}\left(\vec{x}_{1}-\vec{x}_{1}\right)+G_{\sigma_{2}}\left(\vec{x}_{1}-\vec{x}_{2}\right)}{\rho}\right)\right) \vec{v}_{0}+\exp \left(-\frac{\sum_{j=1}^{N} G_{\sigma_{2}}\left(\vec{x}_{i}-\vec{x}_{j}\right)}{\rho}\right) \vec{g}_{1} \\
& \Rightarrow \\
& \left(1-1+\exp \left(-\frac{\sum_{j=1}^{N} G_{\sigma_{2}}\left(\vec{x}_{i}-\vec{x}_{j}\right)}{\rho}\right)\right) \vec{v}_{0} \\
& =\left(1-\exp \left(-\frac{\sum_{j=1}^{N} G_{\sigma_{2}}\left(\vec{x}_{i}-\vec{x}_{j}\right)}{\rho}\right)\right)\left(\sum_{j=1}^{N} H_{\sigma_{1}}\left(\vec{x}_{i}-\vec{x}_{j}\right)+c_{a} \sum_{j=1}^{N} K_{\sigma_{3}}\left(\vec{x}_{i}-\vec{x}_{j}\right)\right) \\
& +\exp \left(-\frac{\sum_{j=1}^{N} G_{\sigma_{2}}\left(\vec{x}_{i}-\vec{x}_{j}\right)}{\rho}\right) \vec{g}_{1} \\
& \Rightarrow \\
& \exp \left(-\frac{\sum_{j=1}^{N} G_{\sigma_{2}}\left(\vec{x}_{i}-\vec{x}_{j}\right)}{\rho}\right)\left(\vec{v}_{0}-\vec{g}_{1}\right) \\
& =\left(1-\exp \left(-\frac{\sum_{j=1}^{N} G_{\sigma_{2}}\left(\vec{x}_{i}-\vec{x}_{j}\right)}{\rho}\right)\right)\left(\sum_{j=1}^{N} H_{\sigma_{1}}\left(\vec{x}_{i}-\vec{x}_{j}\right)+c_{a} \sum_{j=1}^{N} K_{\sigma_{3}}\left(\vec{x}_{i}-\vec{x}_{j}\right)\right) \\
& \Rightarrow \\
& \vec{v}_{0}-\vec{g}_{1}=\left(\exp \left(\frac{\sum_{j=1}^{N} G_{\sigma_{2}}\left(\vec{x}_{i}-\vec{x}_{j}\right)}{\rho}\right)-1\right)\left(\sum_{j=1}^{N} H_{\sigma_{1}}\left(\vec{x}_{i}-\vec{x}_{j}\right)+c_{a} \sum_{j=1}^{N} K_{\sigma_{3}}\left(\vec{x}_{i}-\vec{x}_{j}\right)\right)
\end{aligned}
$$


Finally, we get:

$$
\begin{aligned}
\vec{g}_{1}= & \vec{v}_{0}-\left(\exp \left(\frac{\sum_{j=1}^{N} G_{\sigma_{2}}\left(\vec{x}_{i}-\vec{x}_{j}\right)}{\rho}\right)-1\right)\left(\sum_{j=1}^{N} H_{\sigma_{1}}\left(\vec{x}_{i}-\vec{x}_{j}\right)+c_{a} \sum_{j=1}^{N} K_{\sigma_{3}}\left(\vec{x}_{i}-\vec{x}_{j}\right)\right) \\
= & \vec{v}_{0}-\left(\exp \left(\frac{G_{\sigma_{2}}\left(\vec{x}_{1}-\vec{x}_{1}\right)+G_{\sigma_{2}}\left(\vec{x}_{1}-\vec{x}_{2}\right)}{\rho}\right)-1\right) \\
& \times\left(H_{\sigma_{1}}\left(\vec{x}_{1}-\vec{x}_{1}\right)+H_{\sigma_{1}}\left(\vec{x}_{1}-\vec{x}_{2}\right)+c_{a}\left(K_{\sigma_{3}}\left(\vec{x}_{1}-\vec{x}_{1}\right)+K_{\sigma_{3}}\left(\vec{x}_{1}-\vec{x}_{2}\right)\right)\right)
\end{aligned}
$$

$H$ and $K$ are odd functions, so $H_{\sigma_{1}}\left(\vec{x}_{1}-\vec{x}_{1}\right)=K_{\sigma_{3}}\left(\vec{x}_{1}-\vec{x}_{1}\right)=0$. So we get:

$$
\vec{g}_{1}=\vec{v}_{0}-\left(\exp \left(\frac{G_{\sigma_{2}}\left(\vec{x}_{1}-\vec{x}_{1}\right)+G_{\sigma_{2}}\left(\vec{x}_{1}-\vec{x}_{2}\right)}{\rho}\right)-1\right)\left(H_{\sigma_{1}}\left(\vec{x}_{1}-\vec{x}_{2}\right)+c_{a} K_{\sigma_{3}}\left(\vec{x}_{1}-\vec{x}_{2}\right)\right)
$$

Similarly, for $\vec{g}_{2}$, we get:

$$
\vec{g}_{2}=\vec{v}_{0}-\left(\exp \left(\frac{G_{\sigma_{2}}\left(\vec{x}_{2}-\vec{x}_{1}\right)+G_{\sigma_{2}}\left(\vec{x}_{2}-\vec{x}_{2}\right)}{\rho}\right)-1\right)\left(H_{\sigma_{1}}\left(\vec{x}_{2}-\vec{x}_{1}\right)+c_{a} K_{\sigma_{3}}\left(\vec{x}_{2}-\vec{x}_{1}\right)\right)
$$

We could find that (3.6) and (3.7) is the discretized form of (3.4) and (3.5).

Now we take a further look into the discretized model. Remember, the functions of $H, G, K$ are in the following form:

$$
\begin{aligned}
& H_{\sigma_{1}}=\frac{1}{8 \pi \sigma_{1}^{4}} \vec{x} \exp \left(-\frac{|\vec{x}|^{2}}{4 \sigma_{1}^{2}}\right) \quad \text { (repulsion) } \\
& G_{\sigma_{2}}=\frac{1}{4 \pi \sigma_{2}^{2}} \exp \left(-\frac{|\vec{x}|^{2}}{4 \sigma_{2}^{2}}\right) \quad \text { (orientation) } \\
& K_{\sigma_{3}}=-\frac{1}{64 \pi \sigma_{3}^{6}} \vec{x}|\vec{x}|^{2} \exp \left(-\frac{|\vec{x}|^{2}}{4 \sigma_{3}^{2}}\right) \quad \text { (attraction) }
\end{aligned}
$$

$H$ and $K$ are odd functions, and $\mathrm{G}$ is an even function, so

$$
\begin{aligned}
& H_{\sigma_{1}}\left(\vec{x}_{1}-\vec{x}_{2}\right)=-H_{\sigma_{1}}\left(\vec{x}_{2}-\vec{x}_{1}\right) \\
& K_{\sigma_{3}}\left(\vec{x}_{1}-\vec{x}_{2}\right)=-K_{\sigma_{3}}\left(\vec{x}_{2}-\vec{x}_{1}\right) \\
& G_{\sigma_{2}}\left(\vec{x}_{1}-\vec{x}_{2}\right)=G_{\sigma_{2}}\left(\vec{x}_{2}-\vec{x}_{1}\right) \\
& G_{\sigma_{2}}\left(\vec{x}_{1}-\vec{x}_{1}\right)=G_{\sigma_{2}}\left(\vec{x}_{2}-\vec{x}_{2}\right)
\end{aligned}
$$


From (3.6) and (3.7), using the relationship (3.8), we could get:

$$
\vec{g}_{1}-\vec{g}_{2}=-2\left(\exp \left(\frac{G_{\sigma_{2}}\left(\vec{x}_{1}-\vec{x}_{1}\right)+G_{\sigma_{2}}\left(\vec{x}_{1}-\vec{x}_{2}\right)}{\rho}\right)-1\right)\left(H_{\sigma_{1}}\left(\vec{x}_{1}-\vec{x}_{2}\right)+c_{a} K_{\sigma_{3}}\left(\vec{x}_{1}-\vec{x}_{2}\right)\right)
$$

If the initial positions of the two individuals are symmetric with respect to the $\mathrm{x}$ axis, and the external information is also symmetric with respect to the $\mathrm{x}$-axis, then the interactions between these two individuals could be described by Figure 3.10. We could suppose that the stable configuration should satisfy the following three conditions:

1. The direction of the velocity should be horizontal.

2. $x_{1}=x_{2}$.

3. $y_{1}=-y_{2}$.

where $\vec{x}_{1}=\left(x_{1}, y_{1}\right), \vec{x}_{2}=\left(x_{2}, y_{2}\right), \vec{g}_{1}=\left(g_{1, x}, g_{1, y}\right)$, and $\vec{g}_{2}=\left(g_{2, x}, g_{2, y}\right)$.

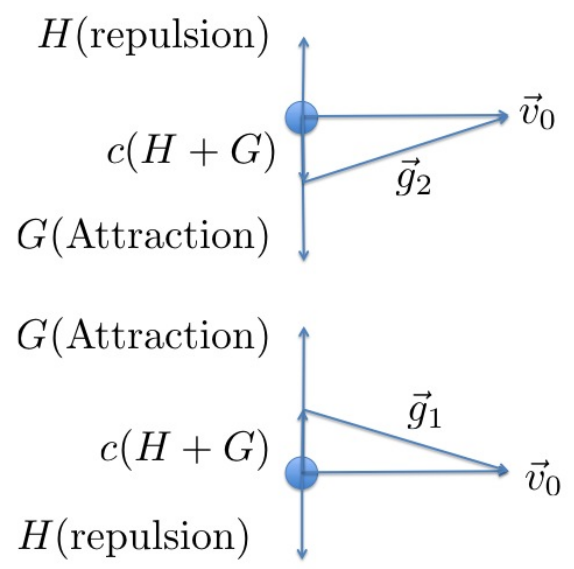

Figure 3.10: The relationship between local interactions and external information at steady state. 
We combine (3.9) with the three conditions that the stationary configuration should meet, we get the following equations:

$$
\begin{aligned}
& g_{1, x}-g_{2, x}=-2\left(\exp \left(\frac{\frac{1}{4 \pi \sigma_{2}^{2}} \exp \left(-\frac{\left(x_{1}-x_{1}\right)^{2}+\left(y_{1}-y_{1}\right)^{2}}{4 \sigma_{2}^{2}}\right)+\frac{1}{4 \pi \sigma_{2}^{2}} \exp \left(-\frac{\left(x_{1}-x_{2}\right)^{2}+\left(y_{1}-y_{2}\right)^{2}}{4 \sigma_{2}^{2}}\right)}{\rho}\right)-1\right) \\
& \times\left(\frac{1}{8 \pi \sigma_{1}^{4}}\left(x_{1}-x_{2}\right) \exp \left(-\frac{\left(x_{1}-x_{2}\right)^{2}+\left(y_{1}-y_{2}\right)^{2}}{4 \sigma_{1}^{2}}\right)\right. \\
& \left.-c_{a} \frac{1}{64 \pi \sigma_{3}^{6}}\left(x_{1}-x_{2}\right)\left(\left(x_{1}-x_{2}\right)^{2}+\left(y_{1}-y_{2}\right)^{2}\right) \exp \left(-\frac{\left(x_{1}-x_{2}\right)^{2}+\left(y_{1}-y_{2}\right)^{2}}{4 \sigma_{3}^{2}}\right)\right) \\
& =0 \quad\left(\text { Since } x_{1}=x_{2}\right) \\
& g_{1, y}-g_{2, y}=-2\left(\exp \left(\frac{\frac{1}{4 \pi \sigma_{2}^{2}} \exp \left(-\frac{\left(x_{1}-x_{1}\right)^{2}+\left(y_{1}-y_{1}\right)^{2}}{4 \sigma_{2}^{2}}\right)+\frac{1}{4 \pi \sigma_{2}^{2}} \exp \left(-\frac{\left(x_{1}-x_{2}\right)^{2}+\left(y_{1}-y_{2}\right)^{2}}{4 \sigma_{2}^{2}}\right)}{\rho}\right)-1\right) \\
& \times\left(\frac{1}{8 \pi \sigma_{1}^{4}}\left(y_{1}-y_{2}\right) \exp \left(-\frac{\left(x_{1}-x_{2}\right)^{2}+\left(y_{1}-y_{2}\right)^{2}}{4 \sigma_{1}^{2}}\right)\right. \\
& \left.-c_{a} \frac{1}{64 \pi \sigma_{3}^{6}}\left(y_{1}-y_{2}\right)\left(\left(x_{1}-x_{2}\right)^{2}+\left(y_{1}-y_{2}\right)^{2}\right) \exp \left(-\frac{\left(x_{1}-x_{2}\right)^{2}+\left(y_{1}-y_{2}\right)^{2}}{4 \sigma_{3}^{2}}\right)\right) \\
& =-2\left(\exp \left(\frac{\frac{1}{4 \pi \sigma_{2}^{2}}+\frac{1}{4 \pi \sigma_{2}^{2}} \exp \left(-\frac{y_{1}^{2}}{\sigma_{2}^{2}}\right)}{\rho}\right)-1\right) \\
& \times\left(\frac{1}{8 \pi \sigma_{1}^{4}} 2 y_{1} \exp \left(-\frac{y_{1}^{2}}{\sigma_{1}^{2}}\right)-c_{a} \frac{1}{64 \pi \sigma_{3}^{6}} 2 y_{1}\left(4 y_{1}^{2}\right) \exp \left(-\frac{y_{1}^{2}}{\sigma_{3}^{2}}\right)\right) \\
& =-\left(\exp \left(\frac{\frac{1}{4 \pi \sigma_{2}^{2}}+\frac{1}{4 \pi \sigma_{2}^{2}} \exp \left(-\frac{y_{1}^{2}}{\sigma_{2}^{2}}\right)}{\rho}\right)-1\right) \\
& \times\left(\frac{1}{2 \pi \sigma_{1}^{4}} y_{1} \exp \left(-\frac{y_{1}^{2}}{\sigma_{1}^{2}}\right)-c_{a} \frac{1}{4 \pi \sigma_{3}^{6}} y_{1}^{3} \exp \left(-\frac{y_{1}^{2}}{\sigma_{3}^{2}}\right)\right)
\end{aligned}
$$

Equation (3.10) holds naturally, since external information are symmetric with respect to $\mathrm{x}$-axis, meaning that they have the same $\mathrm{x}$ coordinate. So we only need to solve (3.11).

We aim to look for a numerical solution for (3.11). Remember, the value of the parameters in equation (3.11) are listed in Table 3.1:

We want to solve for $y_{1}$ which is the $y$-coordinate of the first individual that is below the x-axis. That means we want to find a negative solution. The left hand side of (3.11) (we will call the function on the left hand side as $f_{\text {left }}$ ) represents the difference 
Table 3.1: Parameter values

\begin{tabular}{|c|c|}
\hline \hline$\sigma_{1}$ & $\frac{3}{4}$ \\
\hline$\sigma_{2}$ & $\frac{9}{4}$ \\
\hline$\sigma_{3}$ & $\frac{15}{4}$ \\
\hline$\rho$ & $\frac{6}{4 \pi(9 / 4)^{2}}$ \\
\hline$c_{a}$ & 10 \\
\hline \hline
\end{tabular}

between the two types of external information. $\vec{g}_{1}$ is the external information given to leader 1 , which has a negative y-direction. In reverse, $\vec{g}_{2}$ has a positive y-direction. So $f_{\text {left }}$ is also negative. In order to make this equation hold true, the value of the right hand side of (3.11) (we will call the function on the right hand side as $f_{\text {right }}$ ) should be negative.

One way to solve the equation (3.11) is to find the intersections of the two graphs: the graph of $f_{\text {left }}$, which is a horizontal line, and the graph of $f_{\text {right }}$, which is a function of $y_{1}$. The solution of equation (3.11) is the x-coordinate of the intersection where $y_{1}$ and $f_{\text {right }}$ are both negative. We plot a graph for $f_{\text {right }}$ by plugging the parameters' value into $f_{\text {right }}$.

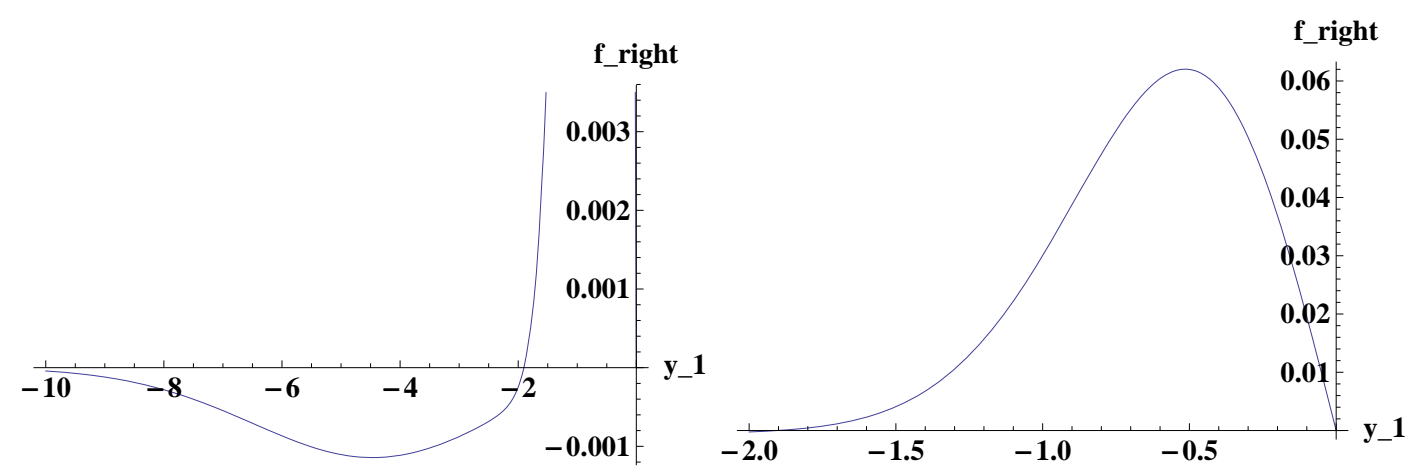

Figure 3.11: Graph of $f_{\text {right }}$ in $(-10,0)$. Details of the graph of $f_{\text {right }}$ in $(-2,0)$ are shown on the right

If we want to find the solution of (3.11), or we want to find at least one intersection in the third quadrant, the graph of $f_{\text {left }}$ should not be below the local minimum 
of $f_{\text {right }}$. Using the function FindMinimum in Mathematica, we found the local minimum is $(-4.46588,-0.00114294)$. That means the maximum difference between the external information's y-coordinate is 0.00114294. In our experiment, we suppose that the external information has unit length, so if a stationary configuration exists, the information differential is less than

$$
2 \arcsin \frac{0.00114294}{2}=0.00114294
$$

So the angle between the external information and the $\mathrm{x}$-axis should be in the interval

$$
(-0.000571469,0.000571469)
$$

From the result above, we find that the swarm is sensitive to the external information. We aim to find the reason. We calculate the weight for external information and the weight for local interaction respectively. The coefficient before the external information for individual 1 is

$$
\exp \left(-\frac{\sum_{j=1}^{N} G_{\sigma_{2}}\left(\vec{x}_{i}-\vec{x}_{j}\right)}{\rho}\right)=\exp \left(-\frac{\frac{1}{4 \pi \sigma_{2}^{2}}+\frac{1}{4 \pi \sigma_{2}^{2}} \exp \left(-\frac{y_{1}^{2}}{\sigma_{2}^{2}}\right)}{\rho}\right)
$$

For $y_{1}$ in $(-10,0)$, we could plot its graph as follows:

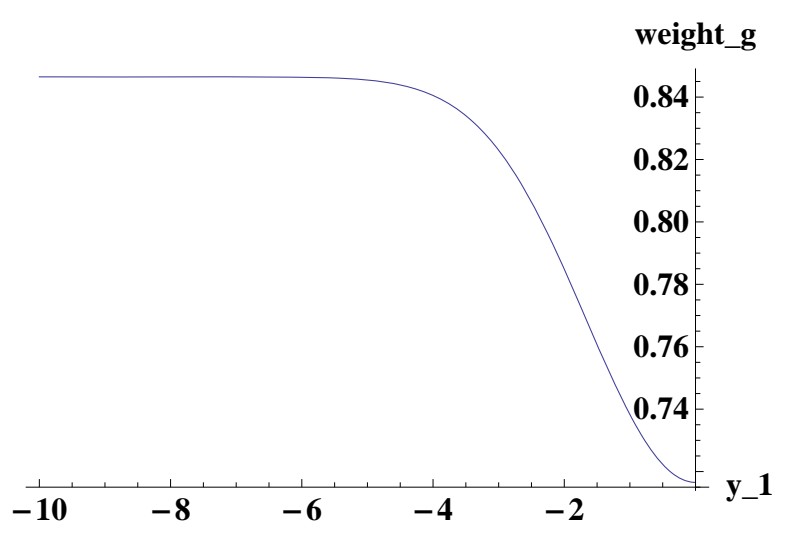

Figure 3.12: Weight for external information when $y_{1}$ is in $(-10,0)$

We find that the weight is in the range of $(0.71,0.85)$. The coefficient before the local interaction for individual 1 is:

$$
1-\exp \left(-\frac{\sum_{j=1}^{N} G_{\sigma_{2}}\left(\vec{x}_{i}-\vec{x}_{j}\right)}{\rho}\right)=1-\exp \left(-\frac{\frac{1}{4 \pi \sigma_{2}^{2}}+\frac{1}{4 \pi \sigma_{2}^{2}} \exp \left(-\frac{y_{1}^{2}}{\sigma_{2}^{2}}\right)}{\rho}\right)
$$


The weight is in the range of $(0.15,0.29)$. From the results, we know that the weight of the local interaction is less than the weight of the external information. But the difference is not that much large. So next, we check the value of the local interaction and compare it with the external information. At the stationary configuration, both of the individuals have the same $\mathrm{x}$-coordinate, so the interaction in the $\mathrm{x}$-direction is equal to 0 . Since the y-component of the velocity is equal to 0 , the interaction in the y direction for individual 1 can be expressed as follows:

$$
\sum_{j=1}^{N} H_{\sigma_{1}}\left(y_{i}-y_{j}\right)+c_{a} \sum_{j=1}^{N} K_{\sigma_{3}}\left(y_{i}-y_{j}\right)=\frac{1}{2 \pi \sigma_{1}^{4}} y_{1} \exp \left(-\frac{y_{1}^{2}}{\sigma_{1}^{2}}\right)-c_{a} \frac{1}{4 \pi \sigma_{3}^{6}} y_{1}^{3} \exp \left(-\frac{y_{1}^{2}}{\sigma_{3}^{2}}\right)
$$

Plot the graph when $y_{1}$ is in $(-10,0)$ :

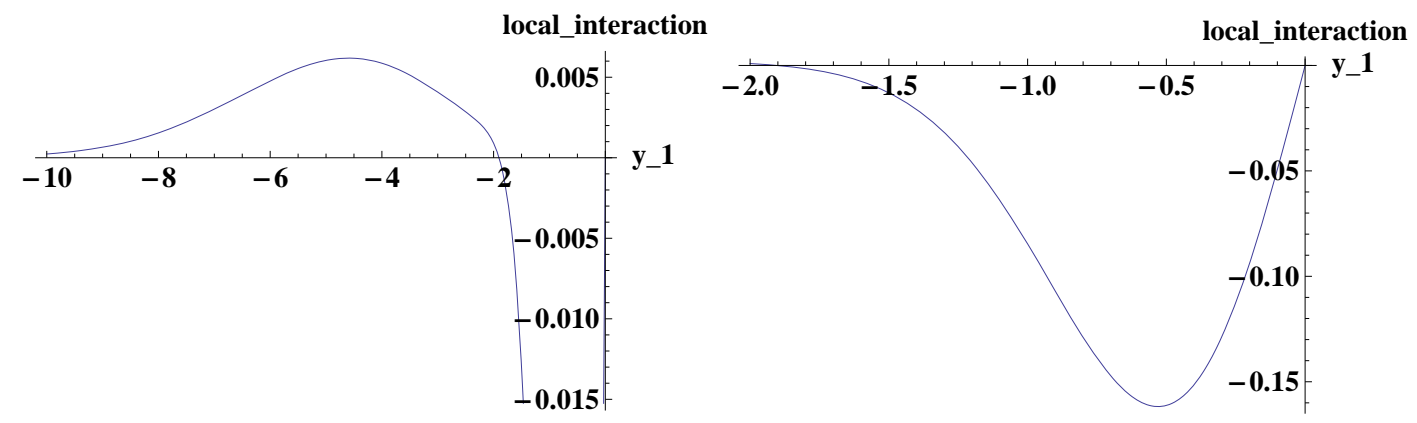

Figure 3.13: Interaction for individual 1 when $y_{1}$ is in $(-10,0)$. Detailed plot of interaction for individual 1 when $y_{1}$ is in $(-2,0)$ is shown on the right.

The interaction is in the range of $(-0.15,0.006)$, which is really small compared to a unit vector, the external information in our experiments. So the swarm is sensitive to the external information.

In order to verify our analysis, we choose a pair of the external information in the range where the stationary configuration exists. If we assume the x-coordinate of the stationary position is 0 , then we could solve (3.11) to get the corresponding ycoordinate. For example, the stationary position is $\vec{x}_{1}=(0,-3)$ and $\vec{x}_{2}=(0,3)$, with respect to the external information $\arg \left(\vec{g}_{1}\right)=-0.00043816$ and $\arg \left(\vec{g}_{2}\right)=0.00043816$. From (3.6) and (3.7), we could get the velocity is equal to $\frac{\vec{g}_{1}+\vec{g}_{2}}{2} \approx(1,0)$. 
But since the system is so sensitive to the external information, a little difference of the external information will make a huge change of the stationary configuration. Due to the error of the computer, if we choose the external information as above, the simulation can not get the stationary configuration as we assume. In this experiment, we set the external information as $\arg \left(\vec{g}_{1}\right)=-0.00043816$ that means $g_{1}$ is below the xaxis and the angle between $g_{1}$ and x-axis is 0.00043816 in radian measure and similarly $\arg \left(\vec{g}_{2}\right)=0.00043816$. We set the initial position as $\left(x_{1}, y_{1}\right)=(-3,0),\left(x_{2}, y_{2}\right)=(3,0)$ and the initial velocity as $\left(v_{x 1}, v_{y 1}\right)=(1,0),\left(v_{x 2}, v_{y 2}\right)=(1,0)$. We choose the time step as 0.1 and the end time as 1000 . We find the the result of our simulation as follows:

Table 3.2: Velocity and position for two individual system

\begin{tabular}{|c|c|c|}
\hline \hline & leader 1 & leader 2 \\
\hline velx & $9.99999904 e-01$ & $9.99999904 e-01$ \\
\hline vely & $4.13011923 e-04$ & $-4.13011923 e-04$ \\
\hline $\mathrm{x}$ & $9.99999904 e+02$ & $9.99999904 e+02$ \\
\hline $\mathrm{y}$ & $-2.25282934 e+00$ & $2.25282934 e+00$ \\
\hline \hline
\end{tabular}

We find that $\mathrm{y}$-coordinate is not equal to 3 or -3 as we expect it to be. Since the velocity in $\mathrm{y}$-direction is not equal to 0 , we know it is not in the steady state.

\subsubsection{Case study II: Extension for two individuals system}

This time we assume that there are two individuals, each with a big mass to represent a group of agents. In our experiments, there are 100 individuals, 50 individuals above the x-axis, and 50 individuals below the x-axis. We use the following equations to represent the density:

$$
\rho(x)=\sum_{i=1}^{2} m \times \delta\left(x-x_{i}\right)
$$


where $m=50$. Similar to (3.6) and (3.7), we could get the discretized model as follows:

$$
\begin{aligned}
\vec{g}_{1}= & \vec{v}_{0}-\left(\exp \left(\frac{m \times G_{\sigma_{2}}\left(\vec{x}_{1}-\vec{x}_{1}\right)+m \times G_{\sigma_{2}}\left(\vec{x}_{1}-\vec{x}_{2}\right)}{\rho}\right)-1\right) \\
& \left(m \times H_{\sigma_{1}}\left(\vec{x}_{1}-\vec{x}_{2}\right)+c_{a} \times m \times K_{\sigma_{3}}\left(\vec{x}_{1}-\vec{x}_{2}\right)\right) \\
\vec{g}_{2}= & \vec{v}_{0}-\left(\exp \left(\frac{m \times G_{\sigma_{2}}\left(\vec{x}_{2}-\vec{x}_{1}\right)+m \times G_{\sigma_{2}}\left(\vec{x}_{2}-\vec{x}_{2}\right)}{\rho}\right)-1\right) \\
& \left(m \times H_{\sigma_{1}}\left(\vec{x}_{2}-\vec{x}_{1}\right)+c_{a} \times m \times K_{\sigma_{3}}\left(\vec{x}_{2}-\vec{x}_{1}\right)\right)
\end{aligned}
$$

(3.13)-(3.14):

$$
\begin{gathered}
\vec{g}_{1}-\vec{g}_{2}=-2\left(\exp \left(\frac{m \times G_{\sigma_{2}}\left(\vec{x}_{1}-\vec{x}_{1}\right)+m \times G_{\sigma_{2}}\left(\vec{x}_{1}-\vec{x}_{2}\right)}{\rho}\right)-1\right) \\
\left(m \times H_{\sigma_{1}}\left(\vec{x}_{1}-\vec{x}_{2}\right)+c_{a} \times m \times K_{\sigma_{3}}\left(\vec{x}_{1}-\vec{x}_{2}\right)\right)
\end{gathered}
$$

Plug in $H_{\sigma 1}, G_{\sigma 2}, K_{\sigma 3}$, then we get:

$$
\begin{aligned}
& g_{1, x}-g_{2, x} \\
= & -2 m\left(\exp \left(\frac{m \times \frac{1}{4 \pi \sigma_{2}^{2}} \exp \left(-\frac{\left(x_{1}-x_{1}\right)^{2}+\left(y_{1}-y_{1}\right)^{2}}{4 \sigma_{2}^{2}}\right)+m \times \frac{1}{4 \pi \sigma_{2}^{2}} \exp \left(-\frac{\left(x_{1}-x_{2}\right)^{2}+\left(y_{1}-y_{2}\right)^{2}}{4 \sigma_{2}^{2}}\right)}{\rho}\right)-1\right) \\
& \times\left(\frac{1}{8 \pi \sigma_{1}^{4}}\left(x_{1}-x_{2}\right) \exp \left(-\frac{\left(x_{1}-x_{2}\right)^{2}+\left(y_{1}-y_{2}\right)^{2}}{4 \sigma_{1}^{2}}\right)\right. \\
& \left.-c_{a} \frac{1}{64 \pi \sigma_{3}^{6}}\left(x_{1}-x_{2}\right)\left(\left(x_{1}-x_{2}\right)^{2}+\left(y_{1}-y_{2}\right)^{2}\right) \exp \left(-\frac{\left(x_{1}-x_{2}\right)^{2}+\left(y_{1}-y_{2}\right)^{2}}{4 \sigma_{3}^{2}}\right)\right) \\
= & 0
\end{aligned}
$$




$$
\begin{aligned}
& g_{1, y}-g_{2, y} \\
= & -2 m\left(\exp \left(\frac{m \times \frac{1}{4 \pi \sigma_{2}^{2}} \exp \left(-\frac{\left(x_{1}-x_{1}\right)^{2}+\left(y_{1}-y_{1}\right)^{2}}{4 \sigma_{2}^{2}}\right)+m \times \frac{1}{4 \pi \sigma_{2}^{2}} \exp \left(-\frac{\left(x_{1}-x_{2}\right)^{2}+\left(y_{1}-y_{2}\right)^{2}}{4 \sigma_{2}^{2}}\right)}{\rho}\right)-1\right) \\
& \times\left(\frac{1}{8 \pi \sigma_{1}^{4}}\left(y_{1}-y_{2}\right) \exp \left(-\frac{\left(x_{1}-x_{2}\right)^{2}+\left(y_{1}-y_{2}\right)^{2}}{4 \sigma_{1}^{2}}\right)\right. \\
& \left.-c_{a} \frac{1}{64 \pi \sigma_{3}^{6}}\left(y_{1}-y_{2}\right)\left(\left(x_{1}-x_{2}\right)^{2}+\left(y_{1}-y_{2}\right)^{2}\right) \exp \left(-\frac{\left(x_{1}-x_{2}\right)^{2}+\left(y_{1}-y_{2}\right)^{2}}{4 \sigma_{3}^{2}}\right)\right) \\
= & -2 m\left(\exp \left(m \frac{\frac{1}{4 \pi \sigma_{2}^{2}}+\frac{1}{4 \pi \sigma_{2}^{2}} \exp \left(-\frac{y_{1}^{2}}{\sigma_{2}^{2}}\right)}{\rho}\right)-1\right) \\
& \times\left(\frac{1}{8 \pi \sigma_{1}^{4}} 2 y_{1} \exp \left(-\frac{y_{1}^{2}}{\sigma_{1}^{2}}\right)-c_{a} \frac{1}{64 \pi \sigma_{3}^{6}} 2 y_{1}\left(4 y_{1}^{2}\right) \exp \left(-\frac{y_{1}^{2}}{\sigma_{3}^{2}}\right)\right) \\
= & \left.-m\left(\exp \left(m \frac{1}{\frac{4 \pi \sigma_{2}^{2}}{4}+\frac{1}{4 \pi \sigma_{2}^{2}} \exp \left(-\frac{y_{1}^{2}}{\sigma_{2}^{2}}\right)}\right)_{\rho}\right)-1\right)\left(\frac{1}{2 \pi \sigma_{1}^{4}} y_{1} \exp \left(-\frac{y_{1}^{2}}{\sigma_{1}^{2}}\right)-c_{a} \frac{1}{4 \pi \sigma_{3}^{6}} y_{1}^{3} \exp \left(-\frac{y_{1}^{2}}{\sigma_{3}^{2}}\right)\right)
\end{aligned}
$$

(3.16) holds naturally. For (3.17), if we know the external information, then we could solve for $y_{1}$, which is the y coordinate of the first individual in the steady state. In our experiment, we choose the mass of each individual as $m=50$. We use $\theta$ to represent the angle between the external information and the $\mathrm{x}$-axis. We choose $\theta$ every $\pi / 18$ in range $(0, \pi / 2)$. The external information is defined as $\vec{g}_{1}=(\cos \theta,-\sin \theta)$ and $\vec{g}_{2}=(\cos \theta, \sin \theta)$. Then we could get the y-equilibrium position listed in Table 3.3.

Table 3.3: y coordinate for the equilibrium state

\begin{tabular}{|c|c|c|c|}
\hline \hline$\theta$ & $y$ & $\theta$ & $y$ \\
\hline 0 & -1.903586 & $\pi / 18$ & -1.903588 \\
\hline $2 \pi / 18$ & -1.903591 & $3 \pi / 18$ & -1.903593 \\
\hline $4 \pi / 18$ & -1.903595 & $5 \pi / 18$ & -1.903597 \\
\hline $6 \pi / 18$ & -1.903598 & $7 \pi / 18$ & -1.903599 \\
\hline $8 \pi / 18$ & -1.903600 & $\pi / 2$ & -1.903600 \\
\hline \hline
\end{tabular}

The velocity is equal to the half of the sum of the external information. 
In order to determine whether the equilibrium is stable or not, we perform the linear stability analysis to see whether the eigenvalue of the linearized matrix is negative or not. If the eigenvalue is negative, then the system is stable. If the eigenvalue is positive, then the system is unstable.

For the two individuals system, we use $\vec{x}_{i}=\left(x_{i}, y_{i}\right)$ to represent the position and $\vec{v}_{i}=\left(v_{i x}, v_{i y}\right)$ to represent the velocity with $i=1,2$, representing the two individuals respectively. The system could be represented as follows, which is a concise form of (1.4). The first two represent the change of the position, and the last two represent the change of the velocity:

$$
\begin{aligned}
\frac{d x_{i}}{d t} & =v_{i x}:=f_{i x} \\
\frac{d y_{i}}{d t} & =v_{i y}:=f_{i y} \\
\frac{d v_{i x}}{d t} & =\kappa\left(v_{d i x}-v_{i x}\right):=g_{i x} \\
\frac{d v_{i y}}{d t} & =\kappa\left(v_{d i y}-v_{i y}\right):=g_{i y}
\end{aligned}
$$

We use $\vec{x}_{i}^{*}, \vec{v}_{i}^{*}(i=1,2)$ to represent the position and velocity of individual $i$ at the steady state, $\vec{x}_{i}^{1}, \vec{v}_{i}^{1}(i=1,2)$ to represent a small disturbance to the position and velocity. We want to find how the disturbance will evolve: whether the disturbance will decay or blow up. The right hand side of (3.18) can be seen as functions of $\vec{v}_{j}$ and $\vec{x}_{j}(j=1,2)$. Then there are 8 variables: $x_{1}, x_{2}, y_{1}, y_{2}, v_{1 x}, v_{2 x}, v_{1 y}, v_{2 y}$ in our system and we have $\vec{x}_{j}=\vec{x}_{j}^{*}+\vec{x}_{j}^{1}, \vec{v}_{j}=\vec{v}_{j}^{*}+\vec{v}_{j}^{1}$. So :

$$
\begin{aligned}
\frac{d\left(\vec{x}_{i}^{*}+\vec{x}_{i}^{1}\right)}{d t} & =\frac{d \vec{x}_{i}^{*}}{d t}+\frac{d \vec{x}_{i}^{1}}{d t}=\vec{v}_{i}^{*}+\frac{d \vec{x}_{i}^{1}}{d t} \\
\vec{f}_{i}\left(\vec{x}_{j}^{*}+\vec{x}_{j}^{1}, \vec{v}_{j}^{*}+\vec{v}_{j}^{1}\right) & =\vec{f}_{i}\left(\vec{x}_{j}^{*}, \vec{v}_{j}^{*}\right)+\vec{f}_{i}^{\prime}\left(\vec{x}_{j}^{*}, \vec{v}_{j}^{*}\right)\left(\begin{array}{c}
\vec{x}_{j}^{1} \\
\vec{v}_{j}^{1}
\end{array}\right)+\cdots
\end{aligned}
$$

From $(3.18 \mathrm{a})$ and $(3.18 \mathrm{~b})$, we know that $\vec{v}_{i}^{*}=\vec{f}_{i}\left(\vec{x}_{j}^{*}, \vec{v}_{j}^{*}\right)$. So

$$
\frac{d \vec{x}_{i}^{1}}{d t}=\vec{f}_{i}^{\prime}\left(\vec{x}_{j}^{*}, \vec{v}_{j}^{*}\right)\left(\begin{array}{c}
\vec{x}_{j}^{1} \\
\vec{v}_{j}^{1}
\end{array}\right)
$$


Similarly,

$$
\begin{gathered}
\frac{d\left(\vec{v}_{i}^{*}+\vec{v}_{i}^{1}\right)}{d t}=\frac{d \vec{v}_{i}^{*}}{d t}+\frac{d \vec{v}_{i}^{1}}{d t}=0+\frac{d \vec{v}_{i}^{1}}{d t} \\
\vec{g}_{i}\left(\vec{x}_{j}^{*}+\vec{x}_{j}^{1}, \vec{v}_{j}^{*}+\vec{v}_{j}^{1}\right)=\vec{g}_{i}\left(\vec{x}_{j}^{*}, \vec{v}_{j}^{*}\right)+\vec{g}_{i}^{\prime}\left(\vec{x}_{j}^{*}, \vec{v}_{j}^{*}\right)\left(\begin{array}{c}
\vec{x}_{j}^{1} \\
\vec{v}_{j}^{1}
\end{array}\right)+\cdots
\end{gathered}
$$

From $(3.18 \mathrm{c})$ and $(3.18 \mathrm{~d})$, we know that $\vec{g}_{i}\left(\vec{x}_{j}^{*}, \vec{v}_{j}^{*}\right)=\overrightarrow{0}$. So we could get

$$
\frac{d \vec{v}_{i}^{1}}{d t}=\vec{g}_{i}^{\prime}\left(\vec{x}_{j}^{*}, \vec{v}_{j}^{*}\right)\left(\begin{array}{c}
\vec{x}_{j}^{1} \\
\vec{v}_{j}^{1}
\end{array}\right)
$$

So far we have two equations (3.19) and (3.20) to describe the evolution of the disturbance. In order to find whether the disturbance will decay or not, we calculate the Jacobian matrix for $\vec{f}$ and $\vec{g}$ to get a linearized system as follows:

$$
\frac{d}{d t}\left(\begin{array}{c}
x_{1} \\
x_{2} \\
y_{1} \\
y_{2} \\
v_{1 x} \\
v_{2 x} \\
v_{1 y} \\
v_{2 y}
\end{array}\right)=\underbrace{\left(\begin{array}{ccccccccc}
\frac{\partial f_{1 x}}{\partial x_{1}} & \frac{\partial f_{1 x}}{\partial x_{2}} & \frac{\partial f_{1 x}}{\partial y_{1}} & \frac{\partial f_{1 x}}{\partial y_{2}} & \frac{\partial f_{1 x}}{\partial v_{1 x}} & \frac{\partial f_{1 x}}{\partial v_{2 x}} & \frac{\partial f_{1 x}}{\partial v_{1 y}} & \frac{\partial f_{1 x}}{\partial v_{2 y}} \\
\frac{\partial f_{2 x}}{\partial x_{1}} & \frac{\partial f_{2 x}}{\partial x_{2}} & \frac{\partial f_{2 x}}{\partial y_{1}} & \frac{\partial f_{2 x}}{\partial y_{2}} & \frac{\partial f_{2 x}}{\partial v_{1 x}} & \frac{\partial f_{2 x}}{\partial v_{2 x}} & \frac{\partial f_{2 x}}{\partial v_{1 y}} & \frac{\partial f_{2 x}}{\partial v_{2 y}} \\
\frac{\partial f_{1 y}}{\partial x_{1}} & \frac{\partial f_{1 y}}{\partial x_{2}} & \frac{\partial f_{1 y}}{\partial y_{1}} & \frac{\partial f_{1 y}}{\partial y_{2}} & \frac{\partial f_{1 y}}{\partial v_{1 x}} & \frac{\partial f_{1 y}}{\partial v_{2 x}} & \frac{\partial f_{1 y}}{\partial v_{1 y}} & \frac{\partial f_{1 y}}{\partial v_{2 y}} \\
\frac{\partial f_{2 y}}{\partial x_{1}} & \frac{\partial f_{2 y}}{\partial x_{2}} & \frac{\partial f_{2 y}}{\partial y_{1}} & \frac{\partial f_{2 y}}{\partial y_{2}} & \frac{\partial f_{2 y}}{\partial v_{1 x}} & \frac{\partial f_{2 y}}{\partial v_{2 x}} & \frac{\partial f_{2 y}}{\partial v_{1 y}} & \frac{\partial f_{2 y}}{\partial v_{2 y}} \\
\frac{\partial g_{1 x}}{\partial x_{1}} & \frac{\partial g_{1 x}}{\partial x_{2}} & \frac{\partial g_{1 x}}{\partial y_{1}} & \frac{\partial g_{1 x}}{\partial y_{2}} & \frac{\partial g_{1 x}}{\partial v_{1 x}} & \frac{\partial g_{1 x}}{\partial v_{2 x}} & \frac{\partial g_{1 x}}{\partial v_{1 y}} & \frac{\partial g_{1 x}}{\partial v_{2 y}} \\
\frac{\partial g_{2 x}}{\partial x_{1}} & \frac{\partial g_{2 x}}{\partial x_{2}} & \frac{\partial g_{2 x}}{\partial y_{1}} & \frac{\partial g_{2 x}}{\partial y_{2}} & \frac{\partial g_{2 x}}{\partial v_{1 x}} & \frac{\partial g_{2 x}}{\partial v_{2 x}} & \frac{\partial g_{2 x}}{\partial v_{1 y}} & \frac{\partial g_{2 x}}{\partial v_{2 y}} \\
\frac{\partial g_{1 y}}{\partial x_{1}} & \frac{\partial g_{1 y}}{\partial x_{2}} & \frac{\partial g_{1 y}}{\partial y_{1}} & \frac{\partial g_{1 y}}{\partial y_{2}} & \frac{\partial g_{1 y}}{\partial v_{1 x}} & \frac{\partial g_{1 y}}{\partial v_{2 x}} & \frac{\partial g_{1 y}}{\partial v_{1 y}} & \frac{\partial g_{1 y}}{\partial v_{2 y}} \\
\frac{\partial g_{2 y}}{\partial x_{2}} & \frac{\partial g_{2 y}}{\partial y_{1}} & \frac{\partial g_{2 y}}{\partial y_{2}} & \frac{\partial g_{2 y}}{\partial v_{1 x}} & \frac{\partial g_{2 y}}{\partial v_{2 x}} & \frac{\partial g_{2 y}}{\partial v_{1 y}} & \frac{\partial g_{2 y}}{\partial v_{2 y}}
\end{array}\right)}_{M}\left(\begin{array}{c}
x_{1} \\
x_{2} \\
y_{1} \\
y_{2} \\
v_{1 x} \\
v_{2 x} \\
v_{1 y} \\
v_{2 y}
\end{array}\right)
$$

Here we use a simplified notation, $x_{1}$, instead of $x_{1}^{1}$, similarly for $\mathrm{y}$-coordinate and velocity. Plug in the expressions for $\vec{f}$, then we get the value for the first four rows of M:

$$
M(1: 4,:)=\left(\begin{array}{cccccccc}
0 & 0 & 0 & 0 & 1 & 0 & 0 & 0 \\
0 & 0 & 0 & 0 & 0 & 1 & 0 & 0 \\
0 & 0 & 0 & 0 & 0 & 0 & 1 & 0 \\
0 & 0 & 0 & 0 & 0 & 0 & 0 & 1
\end{array}\right)
$$


Next we consider the lower part of M. The discretized form for the leaders' desired velocity is:

$$
\begin{aligned}
& \vec{v}_{l d, i}=\left(1-\exp \left(-\frac{\sum_{j=1}^{N} m \times G_{\sigma_{2}}\left(\vec{x}_{i}-\vec{x}_{j}\right)}{\rho}\right)\right)\left(\sum_{j=1}^{N} m \times H_{\sigma_{1}}\left(\vec{x}_{i}-\vec{x}_{j}\right)\right. \\
& \left.+\frac{\sum_{j=1}^{N} m \times G_{\sigma_{2}}\left(\vec{x}_{i}-\vec{x}_{j}\right) \vec{v}\left(\vec{x}_{j}\right)}{\sum_{j=1}^{N} m \times G_{\sigma_{2}}\left(\vec{x}_{i}-\vec{x}_{j}\right)}+c_{a} \sum_{j=1}^{N} m \times K_{\sigma_{3}}\left(\vec{x}_{i}-\vec{x}_{j}\right)\right) \\
& +\exp \left(-\frac{\sum_{j=1}^{N} m \times G_{\sigma_{2}}\left(\vec{x}_{i}-\vec{x}_{j}\right)}{\rho}\right) \vec{g}_{i} \\
& =\left(1-\exp \left(-\frac{m \frac{1}{4 \pi \sigma_{2}^{2}} \exp \left(-\frac{\left|\vec{x}_{i}-\vec{x}_{1}\right|^{2}}{4 \sigma_{2}^{2}}\right)+m \frac{1}{4 \pi \sigma_{2}^{2}} \exp \left(-\frac{\left|\vec{x}_{i}-\vec{x}_{2}\right|^{2}}{4 \sigma_{2}^{2}}\right)}{\rho}\right)\right) \\
& \left(m \frac{1}{8 \pi \sigma_{1}^{4}}\left(\vec{x}_{i}-\vec{x}_{1}\right) \exp \left(-\frac{\left|\vec{x}_{i}-\vec{x}_{1}\right|^{2}}{4 \sigma_{1}^{2}}\right)+m \frac{1}{8 \pi \sigma_{1}^{4}}\left(\vec{x}_{i}-\vec{x}_{2}\right) \exp \left(-\frac{\left|\vec{x}_{i}-\vec{x}_{2}\right|^{2}}{4 \sigma_{1}^{2}}\right)\right. \\
& +\frac{m \frac{1}{4 \pi \sigma_{2}^{2}} \exp \left(-\frac{\left|\vec{x}_{i}-\vec{x}_{1}\right|^{2}}{4 \sigma_{2}^{2}}\right) \vec{v}_{1}+m \frac{1}{4 \pi \sigma_{2}^{2}} \exp \left(-\frac{\left|\vec{x}_{i}-\vec{x}_{2}\right|^{2}}{4 \sigma_{2}^{2}}\right) \vec{v}_{2}}{m \frac{1}{4 \pi \sigma_{2}^{2}} \exp \left(-\frac{\left|\vec{x}_{i}-\vec{x}_{1}\right|^{2}}{4 \sigma_{2}^{2}}\right)+m \frac{1}{4 \pi \sigma_{2}^{2}} \exp \left(-\frac{\left|\vec{x}_{i}-\vec{x}_{2}\right|^{2}}{4 \sigma_{2}^{2}}\right)} \\
& -c_{a} m \frac{1}{64 \pi \sigma_{3}^{6}}\left(\vec{x}_{i}-\vec{x}_{1}\right)\left|\vec{x}_{i}-\vec{x}_{1}\right|^{2} \exp \left(-\frac{\left|\vec{x}_{i}-\vec{x}_{1}\right|^{2}}{4 \sigma_{3}^{2}}\right) \\
& \left.-c_{a} m \frac{1}{64 \pi \sigma_{3}^{6}}\left(\vec{x}_{i}-\vec{x}_{2}\right)\left|\vec{x}_{i}-\vec{x}_{2}\right|^{2} \exp \left(-\frac{\left|\vec{x}_{i}-\vec{x}_{2}\right|^{2}}{4 \sigma_{3}^{2}}\right)\right) \\
& +\exp \left(-\frac{m \frac{1}{4 \pi \sigma_{2}^{2}} \exp \left(-\frac{\left|\vec{x}_{i}-\vec{x}_{1}\right|^{2}}{4 \sigma_{2}^{2}}\right)+m \frac{1}{4 \pi \sigma_{2}^{2}} \exp \left(-\frac{\left|\vec{x}_{i}-\vec{x}_{2}\right|^{2}}{4 \sigma_{2}^{2}}\right)}{\rho}\right) \vec{g}_{i}
\end{aligned}
$$

So for the first leader:

$$
\begin{aligned}
v_{d, 1 x}= & \left(1-\exp \left(-\frac{m \frac{1}{4 \pi \sigma_{2}^{2}}+m \frac{1}{4 \pi \sigma_{2}^{2}} \exp \left(-\frac{\left(x_{1}-x_{2}\right)^{2}+\left(y_{1}-y_{2}\right)^{2}}{4 \sigma_{2}^{2}}\right)}{\rho}\right)\right) \\
& \left(m \frac{1}{8 \pi \sigma_{1}^{4}}\left(x_{1}-x_{2}\right) \exp \left(-\frac{\left(x_{1}-x_{2}\right)^{2}+\left(y_{1}-y_{2}\right)^{2}}{4 \sigma_{1}^{2}}\right)\right. \\
& +\frac{v_{1 x}+\exp \left(-\frac{\left(x_{1}-x_{2}\right)^{2}+\left(y_{1}-y_{2}\right)^{2}}{4 \sigma_{2}^{2}}\right) v_{2 x}}{1+\exp \left(-\frac{\left(x_{1}-x_{2}\right)^{2}+\left(y_{1}-y_{2}\right)^{2}}{4 \sigma_{2}^{2}}\right)} \\
& \left.-c_{a} m \frac{1}{64 \pi \sigma_{3}^{6}}\left(x_{1}-x_{2}\right)\left(\left(x_{1}-x_{2}\right)^{2}+\left(y_{1}-y_{2}\right)^{2}\right) \exp \left(-\frac{\left(x_{1}-x_{2}\right)^{2}+\left(y_{1}-y_{2}\right)^{2}}{4 \sigma_{3}^{2}}\right)\right) \\
& +\exp \left(-\frac{m \frac{1}{4 \pi \sigma_{2}^{2}}+m \frac{1}{4 \pi \sigma_{2}^{2}} \exp \left(-\frac{\left(x_{1}-x_{2}\right)^{2}+\left(y_{1}-y_{2}\right)^{2}}{4 \sigma_{2}^{2}}\right)}{\rho}\right) g_{1 x}
\end{aligned}
$$




$$
\begin{aligned}
v_{d, 1 y}= & \left(1-\exp \left(-\frac{m \frac{1}{4 \pi \sigma_{2}^{2}}+m \frac{1}{4 \pi \sigma_{2}^{2}} \exp \left(-\frac{\left(x_{1}-x_{2}\right)^{2}+\left(y_{1}-y_{2}\right)^{2}}{4 \sigma_{2}^{2}}\right)}{\rho}\right)\right) \\
& \left(m \frac{1}{8 \pi \sigma_{1}^{4}}\left(y_{1}-y_{2}\right) \exp \left(-\frac{\left(x_{1}-x_{2}\right)^{2}+\left(y_{1}-y_{2}\right)^{2}}{4 \sigma_{1}^{2}}\right)\right. \\
& +\frac{v_{1 y}+\exp \left(-\frac{\left(x_{1}-x_{2}\right)^{2}+\left(y_{1}-y_{2}\right)^{2}}{4 \sigma_{2}^{2}}\right) v_{2 y}}{1+\exp \left(-\frac{\left(x_{1}-x_{2}\right)^{2}+\left(y_{1}-y_{2}\right)^{2}}{4 \sigma_{2}^{2}}\right)} \\
& \left.-c_{a} m \frac{1}{64 \pi \sigma_{3}^{6}}\left(y_{1}-y_{2}\right)\left(\left(x_{1}-x_{2}\right)^{2}+\left(y_{1}-y_{2}\right)^{2}\right) \exp \left(-\frac{\left(x_{1}-x_{2}\right)^{2}+\left(y_{1}-y_{2}\right)^{2}}{4 \sigma_{3}^{2}}\right)\right) \\
& +\exp \left(-\frac{m \frac{1}{4 \pi \sigma_{2}^{2}}+m \frac{1}{4 \pi \sigma_{2}^{2}} \exp \left(-\frac{\left(x_{1}-x_{2}\right)^{2}+\left(y_{1}-y_{2}\right)^{2}}{4 \sigma_{2}^{2}}\right)}{\rho}\right) g_{1 y}
\end{aligned}
$$

And for the second leader $v_{d, 2}$ :

$$
\begin{aligned}
& v_{d, 2 x}=\left(1-\exp \left(-\frac{m \frac{1}{4 \pi \sigma_{2}^{2}}+m \frac{1}{4 \pi \sigma_{2}^{2}} \exp \left(-\frac{\left(x_{1}-x_{2}\right)^{2}+\left(y_{1}-y_{2}\right)^{2}}{4 \sigma_{2}^{2}}\right)}{\rho}\right)\right) \\
& \left(m \frac{1}{8 \pi \sigma_{1}^{4}}\left(x_{2}-x_{1}\right) \exp \left(-\frac{\left(x_{1}-x_{2}\right)^{2}+\left(y_{1}-y_{2}\right)^{2}}{4 \sigma_{1}^{2}}\right)\right. \\
& +\frac{v_{2 x}+\exp \left(-\frac{\left(x_{1}-x_{2}\right)^{2}+\left(y_{1}-y_{2}\right)^{2}}{4 \sigma_{2}^{2}}\right) v_{1 x}}{1+\exp \left(-\frac{\left(x_{1}-x_{2}\right)^{2}+\left(y_{1}-y_{2}\right)^{2}}{4 \sigma_{2}^{2}}\right)} \\
& \left.-c_{a} m \frac{1}{64 \pi \sigma_{3}^{6}}\left(x_{2}-x_{1}\right)\left(\left(x_{1}-x_{2}\right)^{2}+\left(y_{1}-y_{2}\right)^{2}\right) \exp \left(-\frac{\left(x_{1}-x_{2}\right)^{2}+\left(y_{1}-y_{2}\right)^{2}}{4 \sigma_{3}^{2}}\right)\right) \\
& +\exp \left(-\frac{m \frac{1}{4 \pi \sigma_{2}^{2}}+m \frac{1}{4 \pi \sigma_{2}^{2}} \exp \left(-\frac{\left(x_{1}-x_{2}\right)^{2}+\left(y_{1}-y_{2}\right)^{2}}{4 \sigma_{2}^{2}}\right)}{\rho}\right) g_{2 x} \\
& v_{d, 2 y}=\left(1-\exp \left(-\frac{m \frac{1}{4 \pi \sigma_{2}^{2}}+m \frac{1}{4 \pi \sigma_{2}^{2}} \exp \left(-\frac{\left(x_{1}-x_{2}\right)^{2}+\left(y_{1}-y_{2}\right)^{2}}{4 \sigma_{2}^{2}}\right)}{\rho}\right)\right) \\
& \left(m \frac{1}{8 \pi \sigma_{1}^{4}}\left(y_{2}-y_{1}\right) \exp \left(-\frac{\left(x_{1}-x_{2}\right)^{2}+\left(y_{1}-y_{2}\right)^{2}}{4 \sigma_{1}^{2}}\right)\right. \\
& +\frac{v_{2 y}+\exp \left(-\frac{\left(x_{1}-x_{2}\right)^{2}+\left(y_{1}-y_{2}\right)^{2}}{4 \sigma_{2}^{2}}\right) v_{1 y}}{1+\exp \left(-\frac{\left(x_{1}-x_{2}\right)^{2}+\left(y_{1}-y_{2}\right)^{2}}{4 \sigma_{2}^{2}}\right)} \\
& \left.-c_{a} m \frac{1}{64 \pi \sigma_{3}^{6}}\left(y_{2}-y_{1}\right)\left(\left(x_{1}-x_{2}\right)^{2}+\left(y_{1}-y_{2}\right)^{2}\right) \exp \left(-\frac{\left(x_{1}-x_{2}\right)^{2}+\left(y_{1}-y_{2}\right)^{2}}{4 \sigma_{3}^{2}}\right)\right) \\
& +\exp \left(-\frac{m \frac{1}{4 \pi \sigma_{2}^{2}}+m \frac{1}{4 \pi \sigma_{2}^{2}} \exp \left(-\frac{\left(x_{1}-x_{2}\right)^{2}+\left(y_{1}-y_{2}\right)^{2}}{4 \sigma_{2}^{2}}\right)}{\rho}\right) g_{2 y}
\end{aligned}
$$


Plug (3.21), (3.22), (3.23), (3.24) into (3.18c) and (3.18d), then we could calculate the partial derivatives of the acceleration functions, which are the right hand side of $(3.18 \mathrm{c})$ and $(3.18 \mathrm{~d})$. Then we get an expression for every element in M. Plug the steady state Table 3.3 into $\mathrm{M}$, then we could find the eigenvalue of M. For example, when $\theta=\pi / 2$, we could find at the steady state, $y_{1}=-1.9036004037099705$, and velocity equals half of the sum of the external information. Then we could get the eigenvalues of $\mathrm{M}$ as follows: All the eigenvalues except the last two are negative. The Table 3.4: The eigenvalues of $\mathrm{M}$ for $\theta=\pi / 2$

\begin{tabular}{|c|c|c|c|}
\hline \hline$-0.328321+1.18305 i$ & $-0.328321-1.18305 i$ & -0.656639 & $-4.09105 \times 10^{-6}$ \\
\hline$-4.09105 \times 10^{-6}$ & $-3.2729 \times 10^{-6}$ & $1.88593 \times 10^{-12}$ & $1.32137 \times 10^{-19}$ \\
\hline \hline
\end{tabular}

last two are positive, but they are almost zero. This phenomena could be explained as the parallel translation along the velocity of the steady state. We could calculate the eigenvectors corresponding to each of the above eigenvalues to explain this phenomena. For eigenvalue $1.88593 \times 10^{-12}$, the eigenvector is:

$$
\left(\begin{array}{c}
0.704172 \\
0.704172 \\
-0.0643544 \\
-0.0643544 \\
1.32802 \times 10^{-12} \\
1.32802 \times 10^{-12} \\
-1.21376 \times 10^{-13} \\
-1.21371 \times 10^{-13}
\end{array}\right)
$$


For eigenvalue $1.32137 \times 10^{-19}$, the eigenvector is:

$$
\left(\begin{array}{c}
-0.707107 \\
-0.707107 \\
0 \\
0 \\
9.49497 \times 10^{-20} \\
-9.49497 \times 10^{-20} \\
0 \\
0
\end{array}\right)
$$

The solution of the system $\frac{d \vec{s}}{d t}=M \vec{s}$ is in the form

$$
\vec{s}=\sum_{i=1}^{8} c_{i} \vec{r}_{i} e^{\lambda_{i} t}
$$

where $c_{i}$ is any constant, $\lambda_{i}$ is the eigenvalue of $M$, and $\vec{r}_{i}$ is the corresponding eigenvector of $\lambda_{i}$. Recall that in our problem:

$$
\vec{s}=\left(\begin{array}{c}
x_{1} \\
x_{2} \\
y_{1} \\
y_{2} \\
v_{1 x} \\
v_{2 x} \\
v_{1 y} \\
v_{2 y}
\end{array}\right)
$$

The last two eigenvectors works like a translation. It represents a uniform shift in $x$ and $y$ direction. So when $m=50, \theta=\pi / 2$, the swarm is always stable.

Now we change $m$ to see whether there is any unstable case. For $m=18$, $19,20,25,30,35,40,45,50$, we could always find the steady state for each $\theta$ in $\left(0, \frac{\pi}{18}, \frac{2 \pi}{18}, \frac{3 \pi}{18}, \frac{4 \pi}{18}, \frac{5 \pi}{18}, \frac{6 \pi}{18}, \frac{7 \pi}{18}, \frac{8 \pi}{18}, \frac{9 \pi}{18}\right)$, and the eigenvalues are always negative, so 
they are always stable. For $m=17$, when $\theta \geq 7 \pi / 18$, there is no steady state. When $\theta \leq 6 \pi / 18$, the eigenvalues are all negative. For $m \leq 16$, we could also get similar result. To different $m$, there is a different bifurcation point. We could represent them in pairs:

\begin{tabular}{|c|c|c|c|}
\hline \hline$(\mathrm{m}$, bifurcation $)$ & $(\mathrm{m}$, bifurcation $)$ & $(\mathrm{m}$, bifurcation $)$ & $(\mathrm{m}$, bifurcation $)$ \\
\hline$(10, \pi / 18)$ & $(11,2 \times \pi / 18)$ & $(12,2 \times \pi / 18)$ & $(13,2 \times \pi / 18)$ \\
\hline$(14,3 \times \pi / 18)$ & $(15,4 \times \pi / 18)$ & $(16,5 \times \pi / 18)$ & $(17,7 \times \pi / 18)$ \\
\hline \hline
\end{tabular}

Plot it as follows:

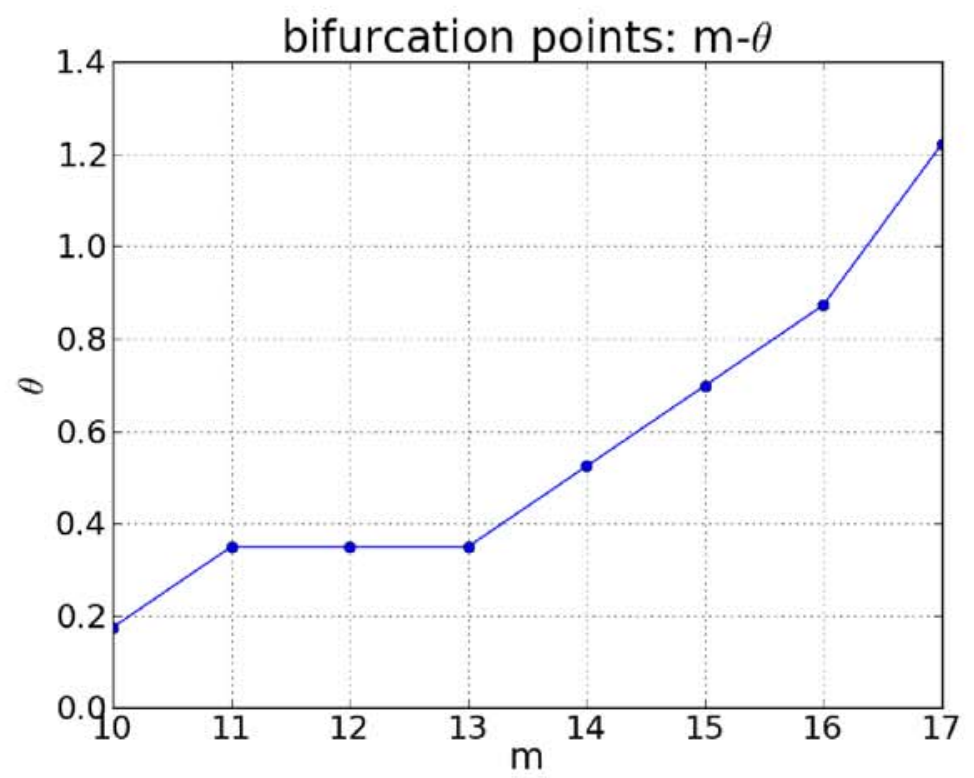

Figure 3.14: Bifurcation point for different $\mathrm{m}$

In order to get a more accurate curve for the relationship between $\mathrm{m}$ and the bifurcation points, we refine our model. Here we choose $\theta$ every $\pi / 36$ and then every $\pi / 72$. Then we get the following plot: 


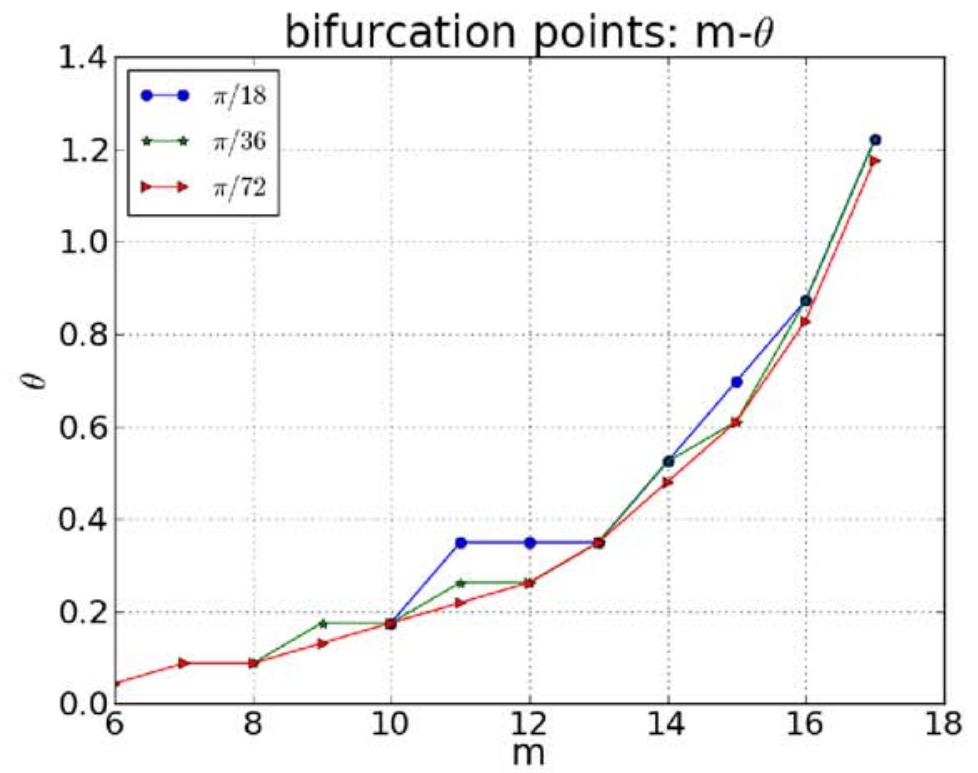

Figure 3.15: Refined Bifurcation point for different $\mathrm{m}$

In order to verify our analytical results, we perform a simulation for two individuals each with a mass equal to $\mathrm{m}$. Remember the density could be represented as follows:

$$
\rho(x)=\sum_{i=1}^{2} m \times \delta\left(x-x_{i}\right)
$$

And the desired velocity for each individual is:

$$
\begin{aligned}
\vec{v}_{l d, i}= & \left(1-\exp \left(-\frac{m \sum_{j=1}^{N} G_{\sigma_{2}}\left(\vec{x}_{i}-\vec{x}_{j}\right)}{\rho}\right)\right)\left(m \sum_{j=1}^{N} H_{\sigma_{1}}\left(\vec{x}_{i}-\vec{x}_{j}\right)\right. \\
& \left.+\frac{\sum_{j=1}^{N} G_{\sigma_{2}}\left(\vec{x}_{i}-\vec{x}_{j}\right) \vec{v}\left(\vec{x}_{j}\right)}{\sum_{j=1}^{N} G_{\sigma_{2}}\left(\vec{x}_{i}-\vec{x}_{j}\right)}+c_{a} m \sum_{j=1}^{N} K_{\sigma_{3}}\left(\vec{x}_{i}-\vec{x}_{j}\right)\right) \\
& +\exp \left(-\frac{m \sum_{j=1}^{N} G_{\sigma_{2}}\left(\vec{x}_{i}-\vec{x}_{j}\right)}{\rho}\right) \vec{g}_{i}
\end{aligned}
$$

Where $\mathrm{N}=2, \mathrm{i}=1,2$.

For $\mathrm{m}=50$, we set the initial velocity as $(1,0)$, the initial position as $(0.5,-0.5)$, $(0.5,0.5)$, and $\theta$ in $(0, \pi / 2)$ every $\pi / 18$. We find that the swarm could always get the 
steady state, and the steady state is stable as we expect it to be. Here we show a case when $\theta=\pi / 2$ :

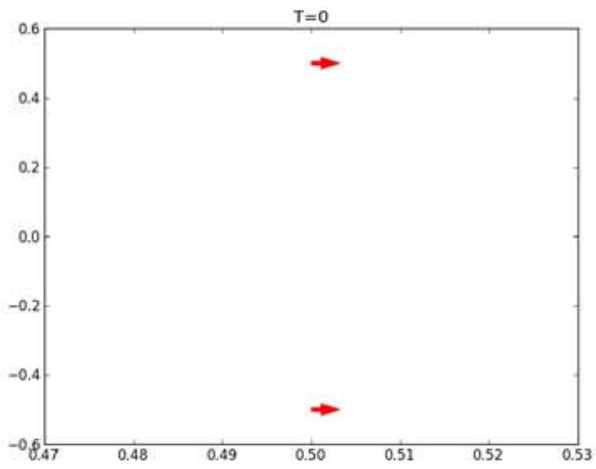

(a) Initial condition

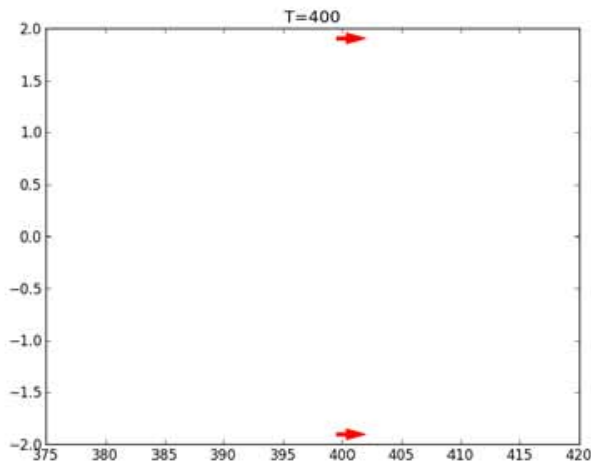

(b) Final state

Figure 3.16: In this swarm, both of them are leaders containing opposite external information, they finally get to a stable steady state.

For $m=17$, we set the same initial condition as $m=50$. And we find that the swarm will get to a stable steady state when $\theta \leq 6 \pi / 18$, and be unstable when $\theta \geq$ $7 \pi / 18$ as we expect it to be.

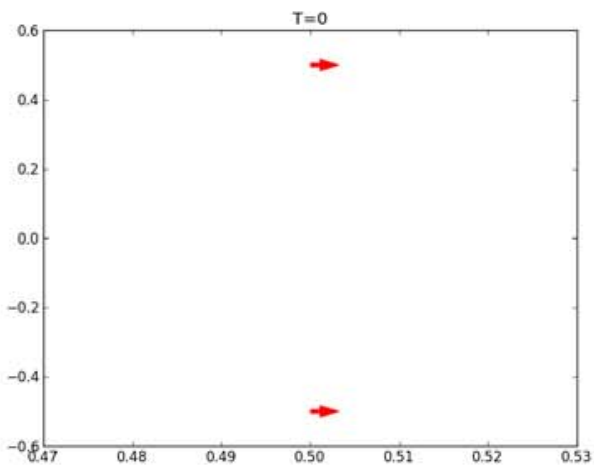

(a) Initial condition

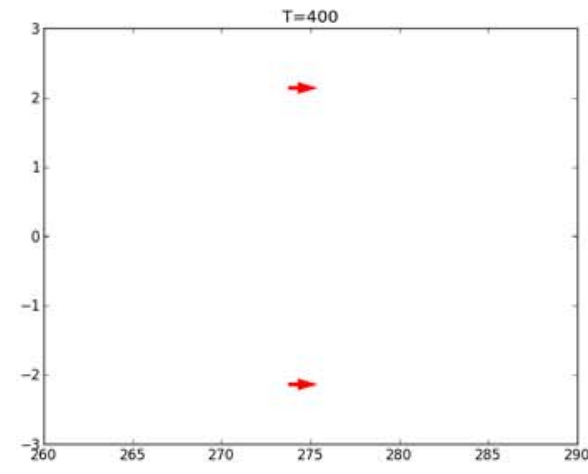

(b) Final state

Figure 3.17: In this swarm, both of them are leaders containing different external information $\theta=6 \pi / 18$ and they finally get to a stable steady state. 


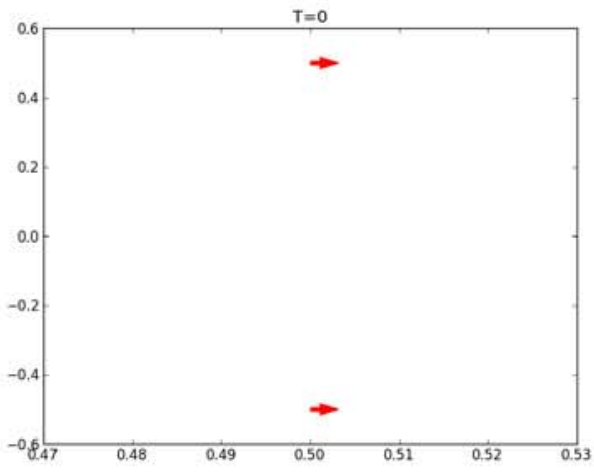

(a) Initial condition

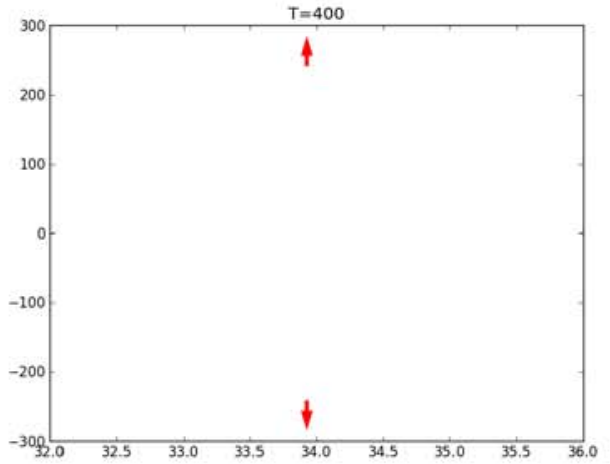

(b) Final state

Figure 3.18: In this swarm, both of them are leaders containing opposite external information, and they finally split.

\subsection{Conclusions}

In this chapter, we explore circumstances where there are two groups of leaders with different additional information. We find that when the information differential is small, the swarm will arrive at a consensus and follow the average desired direction. When the information differential is great, one group of leaders will splinter from the rest of the swarm. All results were validated using simulations with ideal communication and simulations with realistic wireless protocols using QualNet. We also perform two simplified case studies to learn when the bifurcation will happen. Our analytical results are consistent with simulations. 


\section{Chapter 4 INFORMATION TRANSFER IN SWARMS WITH LEADERS}

\subsection{Introduction}

Swarm dynamics is the study of collections of agents that interact with one another without central control. In Chapter 1, we developed a model to describe a swarm with covert leaders. Based on this model, we could determine how the covert leaders affect the motion of the whole group. In this chapter, we consider an inverse problem: Observing a swarm in motion, is it possible to distinguish between followers and leaders? If so, how do we differentiate the leaders from the followers?

Based on our model proposed in Chapter 1, the only difference between the leaders and the followers is that the leaders will respond to the external information. From the simulation performed in Chapter 2, we know that information will spread out through the whole swarm, since we just tell the leaders to go to the right, but eventually the whole swarm went right. We wonder whether the information transfer to the leaders is different from the one to the followers.

Information theory has proven to be a useful framework for the analysis of complex self-organized systems [18]. Mikhail et al. states that the four properties of the self-organization: no external control, an increase in order, robustness and interaction could be interpreted in terms of information dynamics [29]. We could use the principles in information theory to solve the problem in self-organization. He also gave two examples of the application of the information theory in self-organization systems: self-organizing traffic and ant trails. Wang et al. measure information storage and transfer in swarms [41]. The results show us the dynamics within the swarm where we can not tell the process through visualization. Wang et al. also quantify and trace 
information cascades in swarms using the quantities Active Information Storage (AIS) and Transfer Entropy (TE) [40, 22]. This work discovers how an individual's position is related to its information processing role and provides the first information-theoretic evidence that the information cascades occur in waves rippling through the swarm. Due to the powerful tools in information theory and the successful application in swarm, we would like to use theoretical information transfer as a means of analyzing swarm interactions to explore whether or not it is possible to distinguish between followers and leaders based on observations of the swarm.

\subsection{Introduction to Information Theory}

The measurement used to quantify the information should satisfy the following properties:

1. Measure the information locally both in space and time.

2. Figure out the direction of the information transfer so that we could figure out whether the information is transferred from the leaders to the followers or from the followers to the leaders.

3. Be able to separate the information exchanged from input signal or from the history.

Local transfer entropy possesses such kind of property, so we choose it as our measurement. Local transfer entropy is firstly introduced by Lizier et al.[18]. It is derived from transfer entropy. Transfer entropy is also used to quantify the information transfer. It contains inherent directionality as well as explicitly distinguishes information that is actually exchanged from that due to the response to a common input signal or history. But transfer entropy is an average of a sum which is not unified with the specific instances at each specific time. So we discard it.

In order to state local transfer entropy clearly, we will firstly introduce Shannon entropy which is the fundamental quantity of information theory. Then we will give the definition of conditional entropy, relative entropy and mutual information which will be used in the derivation of the local transfer entropy. 
The fundamental quantity in information theory is the Shannon entropy, which is a measure of uncertainty of a random variable, and a measure of the amount of information required on average to describe the random variable. The precise definition is as follows:

Definition 1. Let $X$ be a discrete random variable with alphabet $\mathcal{X}$ and probability mass function $p(x)=\operatorname{Pr}\{X=x\}, x \in \mathcal{X}$. Information content of a particular event $x$ is:

$$
M(x)=-\log p(x)
$$

Then the Shannon entropy $H(X)$ is defined by

$$
H(X)=-\sum_{x \in \mathcal{X}} p(x) \log p(x)
$$

The log is to the base 2 and entropy is expressed in bits.

The following example shows how the entropy represents the uncertainty of a random variable. Let

$$
X= \begin{cases}1 & \text { with probability } p \\ 0 & \text { with probability } 1-p\end{cases}
$$

Then

$$
H(X)=-p \log p-(1-p) \log (1-p):=H(p)
$$

The graph of the function $H(p)$ is shown as Figure 4.1. We find that when $p=0$ or $p=1, H(p)=0$, meaning that the uncertainty is zero. This is compatible with our common sense. Since when $p=0$ or $p=1$, the variable is not random, there is no uncertainty. When $p=\frac{1}{2}, H(p)$ is the largest, meaning that when $p=\frac{1}{2}$, this experiment has the largest uncertainty.

Now we will show that the entropy could also represent the average amount of information to describe the random variable. For any information, we could use a short notation to represent the frequent outcomes, and a relatively long notation to represent 


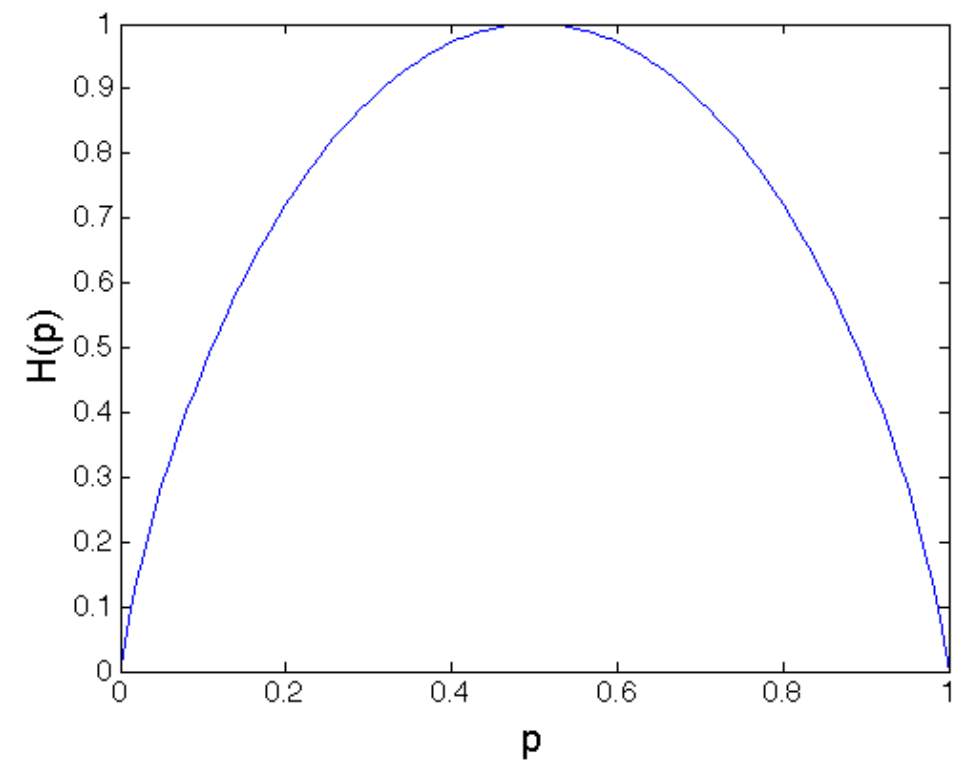

Figure 4.1: $H(p)$ versus $\mathrm{p}$

the less frequent ones. In this way, we construct a code to represent any information we want to tell others.

For example, let $X$ be a random variable with the following distribution and code assignment:

$$
\begin{aligned}
& \operatorname{Pr}(X=1)=\frac{1}{2}, \text { codeword } C(1)=0 \\
& \operatorname{Pr}(X=2)=\frac{1}{4}, \text { codeword } C(2)=10 \\
& \operatorname{Pr}(X=3)=\frac{1}{8}, \text { codeword } C(3)=110 \\
& \operatorname{Pr}(X=4)=\frac{1}{8}, \text { codeword } C(4)=111
\end{aligned}
$$

We find that $X=1$ is the most frequent outcome since the probability of it is equal to a half, then we use a code, 0 , which has length 1 to represent it. In contrast, $X=4$ is the least frequent outcome, so we use a code, 111, which has length 3 to represent it. Then the expected length to describe this random variable will be

$$
\frac{1}{2} * 1+\frac{1}{4} * 2+\frac{1}{8} * 3+\frac{1}{8} * 3=\frac{7}{4}
$$

which is relatively short. 
In the following, we will show that the expected description length must be greater than or equal to the entropy. First, we give the definition of an instantaneous code.

Definition 2. A code is called a prefix code or an instantaneous code if no code word is a prefix of any other codeword.

Here, the prefix could be understood in the following way: In the last example, no code is a prefix of any other codeword. If we just see the code, for example 0 , the outcome must represent 1. But if the codeword for 2 is 01 rather than 10 , then the code 0 will be a prefix for the code 01 . We need to take a look at the second digit to see whether it represents the outcome 1 or the outcome 2. In this case, we need to take 2 digits into consideration to determine the outcome rather than 1 digit. So for outcome 1 , it is a waste of time. But if we coded it just like the example we have shown here, we could determine the outcome instantaneously. That is why it is called instantaneous code.

For an instantaneous code, we have the following properties which will help us to prove that the expected length of codeword is equal to or greater than the Shannon entropy.

Theorem 4.2.1. (Kraft inequality): For any instantaneous code (prefix code) over an alphabet of size $D$, the codeword lengths $l_{1}, l_{2}, \ldots, l_{m}$ must satisfy the inequality

$$
\sum_{i} D^{-l_{i}} \leq 1
$$

Conversely, given a set of codeword lengths that satisfy this inequality, there exists an instantaneous code with these word lengths.

In this theorem, suppose we use binary data to code the outcome, then $D=2$. The codeword length could be different for each outcome, for example, if we use 11 to represent one outcome, then the length of it will be equal to 2 , since there are two digits 1 and 1. The proof for this theorem could be found in [12]. Then if we would 
like to find the prefix code with the minimum expected length, we need to solve a mathematical problem:

Minimize

$$
L=\sum p_{i} l_{i}
$$

over all integers $l_{1}, l_{2}, \ldots, l_{m}$ satisfying

$$
\sum D^{-l_{i}} \leq 1
$$

We want to know the smallest value of $\mathrm{L}$ which is the expected length of the code. First, we just seek a weak solution, where $l_{i}$ does not have to be an integer, and we enforce equality in the constraint. We use the method of Lagrange multipliers to find the minima. Study the Lagrange function defined as:

$$
J=\sum p_{i} l_{i}+\lambda\left(\sum D^{-l_{i}}-1\right)
$$

In order to find the stationary point, we differentiate (4.1) with respect to $l_{i}$ and $\lambda$ :

$$
\begin{aligned}
& \frac{\partial J}{\partial l_{i}}=p_{i}-\lambda D^{-l_{i}} \log _{e} D \\
& \frac{\partial J}{\partial \lambda}=\sum D^{-l_{i}}-1
\end{aligned}
$$

Setting the derivative equal to 0 , we obtain:

$$
\begin{gathered}
D^{-l_{i}}=\frac{p_{i}}{\lambda \log _{e} D} \\
\sum D^{-l_{i}}=1
\end{gathered}
$$

Since $\sum p_{i}=1$, sum (4.4) over i, we find $\lambda=1 / \log _{e} D$ and hence

$$
p_{i}=D^{-l_{i}}
$$

So

$$
l_{i}=-\log _{D} p_{i}
$$


Then the expected codeword length

$$
L=\sum p_{i} l_{i}=-\sum p_{i} \log _{D} p_{i}=H_{D}(X)
$$

But since the $l_{i}$ must be integers, we may not always obtain a minimum. We could choose some codeword length around the point $l_{i}$ to get a relatively shorter descriptive length. The following theorem demonstrates that $l_{i}=-\log _{D} p_{i}$ is a global minimum:

Theorem 4.2.2. The expected length $L$ of any instantaneous D-ary code for a random variable $X$ is greater than or equal to the entropy $H_{D}(X)$, i.e.,

$$
L \geq H_{D}(X)
$$

with equality iff $D^{-l_{i}}=p_{i}$.

The proof of this theorem could be found in [12]. So far, we find that the entropy is the average length of the shortest description of the random variable. This is the reason why we use the entropy to quantify the information exchanged between individuals.

There are some important quantities in information theory which are derived from the Shannon entropy. We will use them to derive the quantity which we use to measure the information transfer in swarm.

The first one is the conditional entropy. During the information transfer, some other individuals may also transfer information, or the information is transferred under some special conditions. We want to eliminate the effect from all the other factors, so we introduce conditional entropy. The conditional entropy of a random variable given another is defined as the expected value of the entropies of the conditional distributions, averaged over the conditioning random variable. Mathematically, if the random 
variable $X$ and $Y$ follow the joint probability $p(x, y)$, i.e. $(X, Y) \sim p(x, y)$, then the conditional entropy $H(Y \mid X)$ is defined as

$$
\begin{aligned}
H(Y \mid X) & =\sum_{x \in \mathcal{X}} p(x) H(Y \mid X=x) \\
& =-\sum_{x \in \mathcal{X}} p(x) \sum_{y \in \mathcal{Y}} p(y \mid x) \log p(y \mid x) \\
& =-\sum_{x \in \mathcal{X}} \sum_{y \in \mathcal{Y}} p(x, y) \log p(y \mid x) \\
& =-E_{p(x, y)} \log p(Y \mid X)
\end{aligned}
$$

where $E_{p(x, y)}$ represents the expected value when the distribution of $(X, Y)$ follows $p(x, y)$.

The relative entropy is a measure of the distance between two distributions. Suppose $p(x)$ and $q(x)$ are two probability mass functions, then the relative entropy or Kullback Leibler distance between these two functions is defined as

$$
\begin{aligned}
D(p \| q) & =\sum_{x \in \mathcal{X}} p(x) \log \frac{p(x)}{q(x)} \\
& =E_{p} \log \frac{p(X)}{q(X)}
\end{aligned}
$$

It is a non-symmetric measure of the difference between two probability distributions $P$ and $Q$. That means $P$ and $Q$ is not interchangeable. This quantity measures the number of extra bits to describe the information which actually follows the $P$ distribution but we suppose it follows the $Q$ distribution. To understand this quantity, from (4.7), we know that for distribution $P$, the optimized code length for event $\mathrm{i}$ is $-\log p_{i}$, and for distribution $Q$, the optimized code length for event $\mathrm{i}$ is $-\log q_{i}$. The samples are chosen from $P$, so it follows the distribution $\left\{p_{i}\right\}$. And we coded it based on $Q$, so for event $\mathrm{i}$, the code length is $-\log q_{i}$. Hence, the average code length would be $-\sum p_{i} \log q_{i}$. But the true average code length is $-\sum p_{i} \log p_{i}$. So the extra bits 
(if we choose base 2) would be

$$
\begin{aligned}
& \left(-\sum p_{i} \log q_{i}\right)-\left(-\sum p_{i} \log p_{i}\right) \\
= & \sum p_{i} \log \frac{p_{i}}{q_{i}} \\
= & D(p \| q)
\end{aligned}
$$

The mutual information is a measure of the amount of information that one random variable contains about another random variable. It measures the reduction of the uncertainty of one random variable when we know another random variable. Mathematically, consider two random variables $X$ and $Y$ with a joint probability mass function $p(x, y)$ and marginal probability mass functions $p(x)$ and $p(y)$. The mutual information $I(X ; Y)$ is the relative entropy between the joint distribution and the product distribution $p(x) p(y)$, i.e.,

$$
\begin{aligned}
I(X ; Y) & =\sum_{x \in \mathcal{X}} \sum_{y \in \mathcal{Y}} p(x, y) \log \frac{p(x, y)}{p(x) p(y)} \\
& =D(p(x, y) \| p(x) p(y)) \\
& =E_{p(x, y)} \log \frac{p(X, Y)}{p(X) p(Y)}
\end{aligned}
$$

We can rewrite the definition of mutual information $I(X ; Y)$ as

$$
\begin{aligned}
I(X ; Y) & =\sum_{x, y} p(x, y) \log \frac{p(x, y)}{p(x) p(y)} \\
& =\sum_{x, y} p(x, y) \log \frac{p(x \mid y)}{p(x)} \\
& =-\sum_{x, y} p(x, y) \log p(x)+\sum_{x, y} p(x, y) \log p(x \mid y) \\
& =-\sum_{x} p(x) \log p(x)-\left(-\sum_{x, y} p(x, y) \log p(x \mid y)\right) \\
& =H(X)-H(X \mid Y)
\end{aligned}
$$


Thus the mutual information $I(X ; Y)$ is the reduction in the uncertainty of $X$ due to the knowledge of $Y$. By symmetry, we can also write the relationship in this way:

$$
I(X ; Y)=H(Y)-H(Y \mid X)
$$

We could also explain the mutual information in this way: We calculate the uncertainty of the two system as though they are independent, but the actual relationship between these variables are $p(x, y)$. Then the uncertainty of the two systems could be expressed as :

$$
H_{2}=-\sum_{x \in \mathcal{X}} \sum_{y \in \mathcal{Y}} p(x, y) \log p(x) q(y)
$$

The actual uncertainty of these two systems is:

$$
H_{1}=-\sum_{x \in \mathcal{X}} \sum_{y \in \mathcal{Y}} p(x, y) \log p(x, y)
$$

So the reduction in the uncertainty of the two systems due to the dependency of these two systems is:

$$
\begin{aligned}
H_{2}-H_{1} & =-\sum_{x \in \mathcal{X}} \sum_{y \in \mathcal{Y}} p(x, y) \log p(x) q(y)-\left(-\sum_{x \in \mathcal{X}} \sum_{y \in \mathcal{Y}} p(x, y) \log p(x, y)\right) \\
& =\sum_{x \in \mathcal{X}} \sum_{y \in \mathcal{Y}} p(x, y) \log \frac{p(x, y)}{p(x) q(y)}
\end{aligned}
$$

which is the mutual information $I(X, Y)$.

The conditional mutual information of random variables $X$ and $Y$ given $Z$ is defined by

$$
\begin{aligned}
I(X ; Y \mid Z) & =H(X \mid Z)-H(X \mid Y, Z) \\
& =E_{p(x, y, z)} \log \frac{p(X, Y \mid Z)}{p(X \mid Z) p(Y \mid Z)}
\end{aligned}
$$

The entropy rate is the rate of growth. In detail, suppose we have a sequence of $n$ random variables, and the entropy rate will measure how the entropy of the sequence grows as $n$ gets larger and larger. Mathematically, the entropy rate of a stochastic process $\left\{X_{i} \mid X_{i} \in \mathcal{X}\right\}$ is defined by

$$
H(\mathcal{X})=\lim _{n \rightarrow \infty} \frac{1}{n} H\left(X_{1}, X_{2}, \ldots, X_{n}\right)
$$


when the limit exists. Here

$$
H\left(X_{1}, X_{2}, \ldots, X_{n}\right)=-\sum_{x_{1} \in X_{1}} \sum_{x_{2} \in X_{2}} \cdots \sum_{x_{n} \in X_{n}} p\left(x_{1}, x_{2}, \cdots, x_{n}\right) \log p\left(x_{1}, x_{2}, \cdots, x_{n}\right)
$$

A related quantity for entropy rate is:

$$
H^{\prime}(\mathcal{X})=\lim _{n \rightarrow \infty} H\left(X_{n} \mid X_{n-1}, X_{n-2}, \ldots, X_{1}\right)
$$

From the formula, we know that $H(\mathcal{X})$ and $H^{\prime}(\mathcal{X})$ represent different entropy rate. $H(\mathcal{X})$ is an average. It represents the contribution from one random variable to the total uncertainty in a system contains $n$ random variables. $H^{\prime}(\mathcal{X})$ is the conditional entropy of the last random variable given the past. A theorem states that for stationary processes both the limits exist and are equal. Formally, we have:

Theorem 4.2.3. For a stationary stochastic process, the limits in (4.14) and (4.16) exist and are equal, i.e.,

$$
H(\mathcal{X})=H^{\prime}(\mathcal{X})
$$

The proof could be found in Cover's book[12].

\subsection{Transfer Entropy}

After knowing the fundamental quantities in information theory, we could begin to derive the quantity we may use in quantifying information transfer. Mutual information has been used as a measure for information transfer in many complex systems [18]. However, mutual information contains no inherent directionality. In order to demonstrate this property, we introduce chain rule firstly.

Theorem 4.3.1. (Chain Rule)

$$
H(X, Y)=H(X)+H(Y \mid X)
$$


Proof.

$$
\begin{aligned}
H(X, Y) & =-\sum_{x \in \mathcal{X}} \sum_{y \in \mathcal{Y}} p(x, y) \log p(x, y) \\
& =-\sum_{x \in \mathcal{X}} \sum_{y \in \mathcal{Y}} p(x, y) \log p(x) p(y \mid x) \\
& =-\sum_{x \in \mathcal{X}} \sum_{y \in \mathcal{Y}} p(x, y) \log p(x)-\sum_{x \in \mathcal{X}} \sum_{y \in \mathcal{Y}} p(x, y) \log p(y \mid x) \\
& =-\sum_{x \in \mathcal{X}} p(x) \log p(x)-\sum_{x \in \mathcal{X}} \sum_{y \in \mathcal{Y}} p(x, y) \log p(y \mid x) \\
& =H(X)+H(Y \mid X)
\end{aligned}
$$

Now we could find

$$
\begin{aligned}
I(X ; Y) & =H(Y)-H(Y \mid X) \\
& =H(Y)-(H(X, Y)-H(X)) \\
& =H(X)+H(Y)-H(X, Y)
\end{aligned}
$$

Therefore, $I(X ; Y)$ is symmetric under the exchange of $X$ and $Y$.

Introducing a time lag in either one of the variables in mutual information can give a directional sense. For example, if we introduce a time lag in $Y$, then we consider $I\left(X_{i}, Y_{i+\tau}\right)$. From (4.12), we know that:

$$
I\left(X_{i}, Y_{i+\tau}\right)=H\left(Y_{i+\tau}\right)-H\left(Y_{i+\tau} \mid X_{i}\right)
$$

Based on the meaning of the mutual information, it represents the reduction of the uncertainty of $Y$ at time $i+\tau$ due to the knowledge of $X$ at time $i$. Or we could say it is the number of bits that could be predicted for $Y$ at time $i+\tau$ by measuring $X$ at time $i$. Similarly, $I\left(Y_{i}, X_{i+\tau}\right)$ is the average number of bits of $X$ at time $t+\tau$ that can be predicted by measuring $Y$ at time $t$. $\mathrm{Xu}$, et at [15] describe $I\left(X_{i}, Y_{i+\tau}\right)$ as the rate of information transmission from variable $X$ to variable $Y$ at a delay of $\tau$. 
But time-lagged mutual information still does not explicitly distinguish the shared information from the exchanged information. For example, if there is a third source $Z$, then the change of $Y$ is due to $Z$, but not $X$. There should be no information transfer between $X$ and $Y$. But since they share the same information, $X$ will give $Y$ a good prediction, then the time-lagged mutual information will still not be equal to zero. To address these inadequacies, Schreiber introduced transfer entropy [33].

Since the transition probability will capture the dynamics of the system, Schreiber consider the transition probability instead of static probability. Consider a system that may be approximated by a stationary Markov process of order $k$, that means the state of the system at time $n+1$ just depends on the last $\mathrm{k}$ states, but not the earlier ones. Mathematically, suppose we use $i_{n+1}$ to represent the state of the system at time $n+1$, then

$$
p\left(i_{n+1} \mid i_{n}, \ldots, i_{n-k+1}\right)=p\left(i_{n+1} \mid i_{n}, \ldots, i_{n-k+1}, i_{n-k}\right)
$$

Here we will use the shorthand notation $i_{n}^{(k)}=\left(i_{n}, \ldots, i_{n-k+1}\right)$ for words of length $k$.

Since it is a stationary stochastic process, based on the Theorem 4.2 .3 , we know that the entropy rate is:

$$
H(\mathcal{X})=H^{\prime}(\mathcal{X})=\lim _{n \rightarrow \infty} H\left(X_{n} \mid X_{n-1}, X_{n-2}, \ldots, X_{1}\right)
$$

Then based on the property of stationary Markov process:

$$
\begin{aligned}
\lim _{n \rightarrow \infty} H\left(X_{n+1} \mid X_{n}, X_{n-1}, \ldots, X_{1}\right) & =\lim _{n \rightarrow \infty} H\left(X_{n+1} \mid X_{n}, X_{n-1}, \ldots, X_{n-k+1}\right) \\
& =H\left(X_{n+1} \mid X_{n}, X_{n-1}, \ldots, X_{n-k+1}\right) \\
& =-\sum p\left(x_{n+1}, x_{n}^{(k)}\right) \log p\left(x_{n+1} \mid x_{n}^{(k)}\right)
\end{aligned}
$$

Just as the way we introduce mutual information, we introduce transfer entropy. Suppose there are two processes $(X, Y)$, we measure the deviation from independence. The only difference is we generalize entropy rate rather than shannon entropy. If the system $Y$ has no influence on $X$, then

$$
p\left(x_{n+1} \mid x_{n}^{(k)}\right)=p\left(x_{n+1} \mid x_{n}^{(k)}, y_{n}^{(l)}\right)
$$


The incorrectness of this independent assumption can again be quantified by the relative entropy (4.10) by which we define the transfer entropy:

$$
T_{Y \rightarrow X}=\sum p\left(x_{n+1}, x_{n}^{(k)}, y_{n}^{(l)}\right) \log \frac{p\left(x_{n+1} \mid x_{n}^{(k)}, y_{n}^{(l)}\right)}{p\left(x_{n+1} \mid x_{n}^{(k)}\right)}
$$

Actually, the transfer entropy can be viewed as a conditional mutual information (4.13). From the formula (4.19), we know that the transfer entropy is the average information contained in the source $Y$ about the next state of the destination $\mathrm{X}$ that was not contained in the past of the destination. Here suppose we use $X^{\prime}$ to represent the next state of the destination $X$. Then mathematically, we could also write the transfer entropy as follows:

$$
T_{Y \rightarrow X}=I\left(Y ; X^{\prime} \mid X\right)=H\left(X^{\prime} \mid X\right)-H\left(X^{\prime} \mid X, Y\right)
$$

\subsection{Local Transfer Entropy}

Transfer entropy represents the information of one source system contained about another destination system regardless of the information already contained in the destination system. It is an average quantity but not the information transferred at each time step. However, sometimes we would like to know the information transfer at each time step between the specific individuals. Lizier et al. [18] derived a local transfer entropy which can quantify the information transfer both in space and time.

From (4.19), we know that the transfer entropy is summed over all possible state transition tuples $u_{n}=\left(x_{n+1}, x_{n}^{(k)}, y_{n}^{(l)}\right)$, weighted by the probability of observing each such tuple. This probability $p\left(u_{n}\right)$ could be calculated from the ratio of the count of such observations $c\left(u_{n}\right)$, to the total number of observations $N$. Mathematically, we could write $p\left(u_{n}\right)=c\left(u_{n}\right) / N$. For $c\left(u_{n}\right)$, we could write it as a sum: $c\left(u_{n}\right)=\sum_{a=1}^{c\left(u_{n}\right)} 1$. 
Then plug the formula $p\left(u_{n}\right)=\left(\sum_{a=1}^{c\left(u_{n}\right)} 1\right) / N$ into (4.19):

$$
\begin{aligned}
T_{Y \rightarrow X} & =\sum_{u_{n}}\left(\frac{1}{N} \sum_{a=1}^{c\left(u_{n}\right)} 1\right) \log \frac{p\left(x_{n+1} \mid x_{n}^{(k)}, y_{n}^{(l)}\right)}{p\left(x_{n+1} \mid x_{n}^{(k)}\right)} \\
& =\frac{1}{N} \sum_{u_{n}} \sum_{a=1}^{c\left(u_{n}\right)} \log \frac{p\left(x_{n+1} \mid x_{n}^{(k)}, y_{n}^{(l)}\right)}{p\left(x_{n+1} \mid x_{n}^{(k)}\right)} \\
& =\frac{1}{N} \sum_{t=1}^{N} \log \frac{p\left(x_{n+1} \mid x_{n}^{(k)}, y_{n}^{(l)}\right)}{p\left(x_{n+1} \mid x_{n}^{(k)}\right)}
\end{aligned}
$$

If we define the local transfer entropy as

$$
t_{Y \rightarrow X}(n+1, k, l)=\log \frac{p\left(x_{n+1} \mid x_{n}^{(k)}, y_{n}^{(l)}\right)}{p\left(x_{n+1} \mid x_{n}^{(k)}\right)}
$$

Then this measure is local in that it is defined at each time $n$ for each destination element $X$ in the system and each causal information source $Y$ of the destination.

Now we could find that the transfer entropy metric is a global average of a local transfer entropy at each observation:

$$
\begin{aligned}
& T_{Y \rightarrow X}=\left\langle t_{Y \rightarrow X}(n+1, k, l)\right\rangle ; \\
& t_{Y \rightarrow X}(n+1, k, l)=\log \frac{p\left(x_{n+1} \mid x_{n}^{(k)}, y_{n}^{(l)}\right)}{p\left(x_{n+1} \mid x_{n}^{(k)}\right)}
\end{aligned}
$$

Here we condition on the past $\mathrm{k}$ states of the destination $X$ to eliminate the information contained in the history of the destination individual. But if the system is not a Markov process of order $\mathrm{k}$, then the self-influence transmitted prior to this k steps will not be eliminated. So Lizer et al.[18] suggest that taking the asymptote $k \rightarrow \infty$ is most correct for agents displaying non-Markovian dynamics. As such, they formalize the local transfer entropy as:

$$
t_{Y \rightarrow X}(n+1, k, l)=\lim _{k \rightarrow \infty} \log \frac{p\left(x_{n+1} \mid x_{n}^{(k)}, y_{n}^{(l)}\right)}{p\left(x_{n+1} \mid x_{n}^{(k)}\right)}
$$

In order to eliminate other influences, like a second source $Z$, we could condition the probability on this source. Mathematically, we could write $I\left(Y ; X^{\prime} \mid X, Z\right)$ ), to eliminate their influence from being mistaken as that of the source $Y$. For Elementary 
Cellular Automata (ECAs), we may condition on the cells in the destination cell's neighborhood to eliminate the influence from those influences. Suppose the destination variable is represented by $x_{i, n+1}$, which means the state of $i$ th cell at time $n+1$. And we want to calculate the information transfer from the source variable $x_{i-j, n}$, which means the state of the $i-j$ th cell at time $n$. Then we use the following sets to represent all the influences from the neighbors and the past of the destination cell:

$$
v_{i, j, n}^{r}=\left\{x_{i+q, n} \mid \forall q:-r \leq q \leq+r, q \neq-j, 0\right\}
$$

where $\mathrm{r}$ is the range of causal information contributors (i.e. the cell range for CAs). We then can derive the information transferred exactly from the source variable:

$$
t_{c}(i, j, n+1)=\lim _{k \rightarrow \infty} \log \frac{p\left(x_{i, n+1} \mid x_{i, n}^{(k)}, x_{i-j, n}, v_{i, j, n}^{r}\right)}{p\left(x_{i, n+1} \mid x_{i, n}^{(k)}, v_{i, j, n}^{r}\right)}
$$

For other information contributor $\mathrm{W}$, we could also condition on $\mathrm{W}$ to eliminate the effect from $\mathrm{W}$ :

$$
t_{Y \rightarrow X \mid W}(n+1, k)=\lim _{k \rightarrow \infty} \log _{2} \frac{p\left(x_{n+1} \mid x_{n}^{(k)}, w_{n}, y_{n}\right)}{p\left(x_{n+1} \mid x_{n}^{(k)}, w_{n}\right)}
$$

\subsection{Transfer Entropy for Swarms}

We would like to apply this measurement (4.21) to swarms. So we need to determine the variables for $x, y, w$. Let $j$ be a particle that is within another particle $i$ 's zones of interaction, so $i$ and $j$ form a causal pair. Our aim is to find what influence does $j$ have on $i$. That means $j$ is the source individual, and $i$ is the destination individual. And we would like to know the amount of information transferred from $j$ to $i$.

We note that if two pairs of particles have the exact same relative positions and velocities, but have different absolute positions and headings, then the information transfer between these two pairs should be the same. As such, we will focus our measurements on the relative positions and relative velocities. And for the destination variables, we will consider the change of the velocity. Suppose we use $\vec{v}_{i}^{n}$ to represent the 
velocity of particle $i$ at time $\mathrm{n}$. Then the destination variable will be $x_{n+1}=\vec{v}_{i}^{n+1}-\vec{v}_{i}^{n}$. If we just consider the past 1 state, this means we use $\mathrm{k}=1$, then the past state of the destination is the change in velocity of $i$ at time step $\mathrm{n}, x_{n}^{(k)}=\vec{v}_{i}^{n}-\vec{v}_{i}^{n-1}$. If we use $\vec{s}_{i}^{n}$ to represent particle $i$ 's position at time $\mathrm{n}$, then we see that the source variable is the relative positions and velocities between the particles at time step n: $y_{n}=\left\{\vec{s}_{i}^{n}-\vec{s}_{j}^{n}, \vec{v}_{i}^{n}-\vec{v}_{j}^{n}\right\}$. We also condition the transfer on the speed of $i$ at $\mathrm{n}$, since the absolute velocity of the particle may have some indirect influence on the change in state (for example by influencing how often the source and destination have recently interacted). So far we define all the variables in (4.21):

$$
\begin{aligned}
& y_{n}=\left\{\vec{s}_{i}^{n}-\vec{s}_{j}^{n}, \vec{v}_{i}^{n}-\vec{v}_{j}^{n}\right\} \\
& w_{n}=\left|\vec{v}_{i}^{n}\right| \\
& x_{n}^{(k)}=\vec{v}_{i}^{n}-\vec{v}_{i}^{n-1} \\
& x_{n+1}=\vec{v}_{i}^{n+1}-\vec{v}_{i}^{n}
\end{aligned}
$$

After determining the variables, we need to figure out a way to calculate the probability distribution function(PDF). Since the swarms are always in motion, a pair of particles, $i$ and $j$ may transfer information at one time step when they are close enough, but may also move outside the interaction zones at next step, then there will be no information transfer between this two individuals. Therefore, counting the observations for each possible tuple to calculate the probability distribution function through a single pair may not give us a good representation of the whole group. Helpfully, in swarm models, we treat the individuals the same. They will respond to the local interactions in the same way. So we can accumulate observations for the probability distribution functions from every transient causal interaction. That is, we count the observations from every transient casual pair. When one particle $j$ is within the interaction zones to have a causal effect over another particle $i$, their interaction is counted for the probability distribution functions, but when $j$ is outside in the interaction zones of $i$, no observation is recorded. Then the probability distribution functions will be applied to any pair if they form a causal relationship at one time step. 
And for our problem since the leaders and followers are known to react differently given their neighboring conditions, and thus the PDF needs to capture the reactions of the individuals within the swarm accordingly. Therefore, we separate the observations by the role of the destination individual, and estimate separate PDFs for those destination individuals that are followers and those that are leaders. Then use the local CTE we could find the information transfer between each individuals.

\subsection{Results}

We characterize overall transfer as the average over all causally connected pairs $Y \rightarrow X^{F}$, where $F$ denotes followers, at each time step:

$$
T(n+1, k)^{F}=\left\langle t_{Y \rightarrow X^{F}}(n+1, k)\right\rangle_{Y \rightarrow X^{F}}
$$

and similarly for the leaders:

$$
T(n+1, k)^{L}=\left\langle t_{Y \rightarrow X^{L}}(n+1, k)\right\rangle_{Y \rightarrow X^{L}}
$$

Then we compare this two values along the time to see whether there is any difference between the leaders and the followers.

We put 100 individuals on a $10 \times 10$ lattice, each with a random initial velocity with speed equal to 1 . We choose $15 \%$ as the leaders and give the leaders external information as $\vec{g}=(1,0)$, that means we want them to go to the right. Driven by the local interaction, the group reorganizes in very short time and then moves to the right as a coherent disk. We calculate the average local transfer entropy both for leaders and followers at each time step. Then we plot the results in Figure 4.2.

We find that though it can be difficult if not impossible to identify leaders through visual inspection, information theory provides an appropriate lens through which this segregation may be possible. (In certain models under certain circumstances, leaders may aggregate at the front, but this is not generally the case.) Using the information theoretic approach, we discard all apriori knowledge of individual interactions and instead use the local CTE in the swarm to see if covert leaders transmit 
or receive more information than followers. While it is apriori intuitive that a leader ought to share more information with the swarm, we find that the inverse is the best way to distinguish covert leaders. A covert leader in a swarm is notable because it receives less information than followers on average. In Fig. 4.2, we can see the average CTE received by the covert leaders is markedly lower than the CTE received by the followers as the swarm organizes itself into a coherent disk.

\subsection{Conclusion}

Information theory provides us a way to distinguish leaders from followers. We find that the leaders will receive less information than the followers. But the disadvantage of this work is that it separates the leaders and followers firstly and then find that there is a difference between these two kinds of individuals. Next we would like to treat the group homogeneously to see whether we could differentiate the leaders from the followers with the help of local conditional transfer entropy. 
Transfer entropy
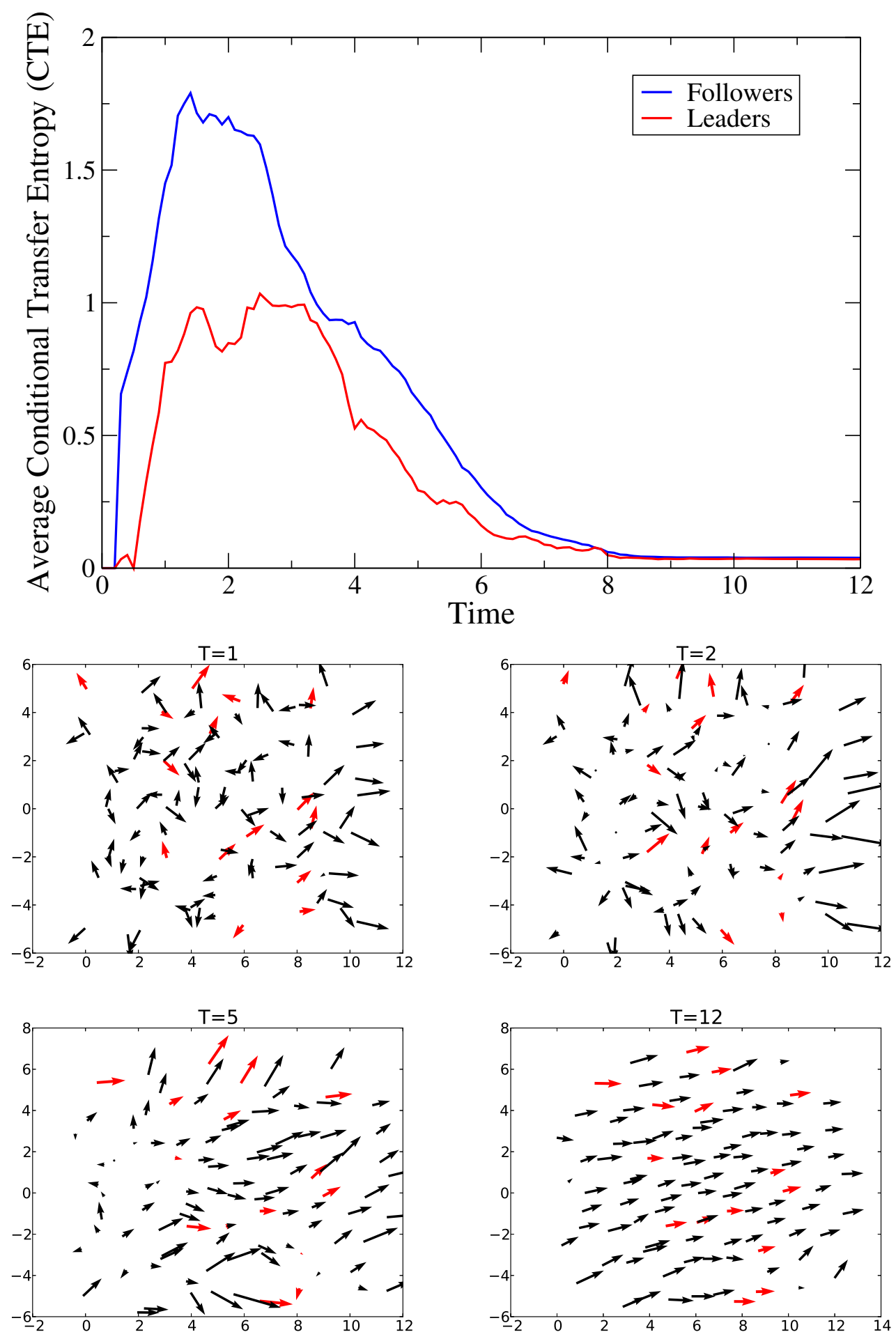

Figure 4.2: The transfer entropy received by followers and leaders over time along with the swarm configuration at key times during self-organization. Leaders are displayed in red, but have the same interaction influence others no differently from the followers. 


\section{Chapter 5 \\ USING THE PAGERANK METHOD TO FIND LEADERS IN A SWARM}

In the previous chapter, we separated the individuals of a swarm based on the different roles of the receivers to calculate the PDF for the leaders and the followers, and then calculated the local transfer entropy (TE). We found that the leaders will receive less local transfer entropy than the followers. This is compatible with our common sense, since if a person already knows where he needs to go, then he will accept less information than someone who does not know where to go.

This result tells us that the local transfer entropy could catch the difference between the leaders and the followers. In this chapter, we still use transfer entropy as a tool to find the leaders. But this time we do not separate the swarm based on the roles of the receivers, since we do not know who are the leaders and who are the followers.

After treating the agents the same, we could calculate the local transfer entropy sent from or received by one individual at each time step. We record the local transfer entropy for each pair of individuals at each time step. For each pair $X$ and $Y$, there are two links between them: from $X$ to $Y$ and from $Y$ to $X$. If we use the local transfer entropy to label the strength of the link, then some of the links are stronger and some of the links are weaker. Given a TE threshold for these links, when the local transfer entropy, for example, from $X$ to $Y$, is smaller than the TE threshold, we delete the link from $X$ to $Y$, then we build a network for the swarm. Inspired by the scale of the Internet, and Google's use of the PageRank method [5] to rank the importance of the web pages, we propose to use the PageRank method to identify the leaders. The underlying assumption for the PageRank method is that more important pages receive 
more links from other pages. From the knowledge accepted from Chapter 4, we know that the leaders would like to receive less transfer entropy than the followers. So we adapt the PageRank method to identify the individuals with less links as the leaders.

\subsection{Introduction to PageRank Method}

PageRank is the method used by Google Search to rank the importance of web pages. The main idea is that the importance of a web page is dependent on the number of pages linked to this page and the importance of these linked pages. For instance, suppose there are four pages linked to each other as shown in Figure 5.1, and we want to measure the importance of these four pages. Here we use the yellow nodes to represent the webpages. The arrows represent the hyperlinks between these web pages. For example, there is an arrow between node 4 and node 2. Based on the direction of the arrow, we know there is a hyperlink in page 4 that will redirect the reader to page 2. Firstly, we may think the node which gets the most references or incoming links

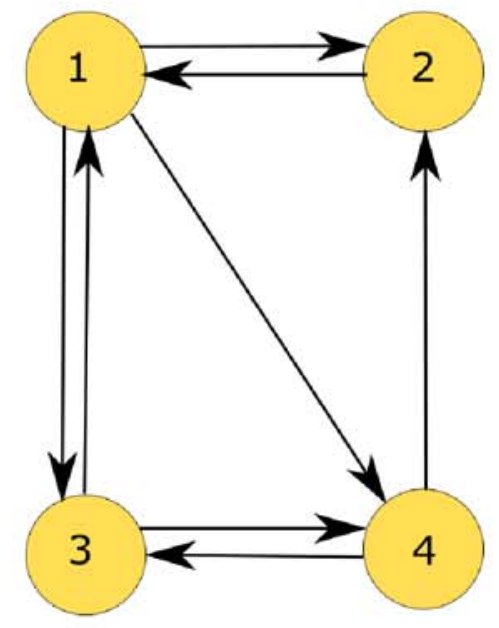

Figure 5.1: Linked pages

from the others to be the most important one. In this way, each node gets 2 references. Any page will be as important as any others. But some nodes contain more outgoing links than others. For example, node 1 contains three outgoing links, but node two only contains one outgoing link. Consider a situation of presidential election. If some residents have two or more votes, then it will not be fair. So we assume that each 
node only has one vote. But some nodes may be more important than others. The important node should have a higher vote weight. By integrating these two factors together, we get a way to evaluate the importance of a node. We use $x_{i}$ to represent the score of page $i$. The larger the score, the higher the rank. Suppose there are $n$ outgoing links from node $i$ to $i_{1}, \ldots, i_{n}$. Then each of the destination nodes will get a score of $\frac{x_{i}}{n}$. The score of one node is the sum of the scores of all of the incoming links - To see this, we use Figure 5.1 as an example. We use $x_{i}(i=1,2,3,4$. $)$ to represent the scores of these four nodes. For node 1, it receives two links from node 2 and node 3 respectively. Node 2 contains only one outgoing link. So node 2 will give its entire vote to node 1 . Node 3 contains two outgoing links. So node 3 will give half of its vote to node 1 and half of its vote to node 4 . The weight of the vote is equal to the score of node 3 . So in total node 1 gets $x_{2}+\frac{1}{2} x_{3}$, which should be equal to the score of node 1. So we obtain the following equation:

$$
x_{1}=x_{2}+\frac{1}{2} x_{3}
$$

Similarly, we obtain the following three equations for nodes 2,3 and 4 , respectively.

$$
\begin{aligned}
x_{2} & =\frac{1}{3} x_{1}+\frac{1}{2} x_{4} \\
x_{3} & =\frac{1}{3} x_{1}+\frac{1}{2} x_{4} \\
x_{4} & =\frac{1}{3} x_{1}+\frac{1}{2} x_{3}
\end{aligned}
$$

We can write the system of equations in matrix form.

$$
\left(\begin{array}{cccc}
0 & 1 & \frac{1}{2} & 0 \\
\frac{1}{3} & 0 & 0 & \frac{1}{2} \\
\frac{1}{3} & 0 & 0 & \frac{1}{2} \\
\frac{1}{3} & 0 & \frac{1}{2} & 0
\end{array}\right)\left(\begin{array}{c}
x_{1} \\
x_{2} \\
x_{3} \\
x_{4}
\end{array}\right)=\left(\begin{array}{c}
x_{1} \\
x_{2} \\
x_{3} \\
x_{4}
\end{array}\right)
$$

Let $A$ represent the coefficient matrix, and $\vec{x}$ represent the unknown vector. Then we need to solve:

$$
A \vec{x}=\vec{x}
$$


This means we need to find the eigenvector of $A$ corresponding to the eigenvalue 1 . Given that matrix $A$ is a column Markov matrix where the sum of each column is equal to 1 , there must be an eigenvalue of $A$ equal to 1 .

Theorem 5.1.1. Let $A$ be an $n \times n$ column Markov matrix, then $A$ must has 1 as its eigenvalue.

Proof. First, we note that $A$ and $A^{T}$ have the same eigenvalues. A short proof of this is as follows. Let $\lambda$ represent the eigenvalue, and $I$ represent the identity matrix. Then

$$
\begin{aligned}
& \operatorname{det}(A-\lambda I) \\
= & \operatorname{det}(A-\lambda I)^{T} \\
= & \operatorname{det}\left(A^{T}-(\lambda I)^{T}\right) \\
= & \operatorname{det}\left(A^{T}-\lambda I\right)
\end{aligned}
$$

So $A$ and $A^{T}$ have the same eigenvalue.

Since $A$ is a column Markov Matrix, the column sun is equal to 1 . So the row sum of $A^{T}$ is equal to one. Suppose we use $e$ to represent the n-dimensional column vector with all components equal to 1 . Then we find that

$$
A^{T} e=e
$$

So 1 is an eigenvalue of $A^{T}$. Therefore, we arrive at the conclusion that 1 is an eigenvalue of $A$.

Thus, we can always solve this linear system. For our sample problem, the eigenvector of $\mathrm{A}$ corresponding to eigenvalue 1 is:

$$
\left(\begin{array}{l}
0.6547 \\
0.4364 \\
0.4364 \\
0.4364
\end{array}\right)
$$


From the result we know that node 1 is the most important page, and all the others are equally important.

However, sometimes the dimension of the eigenspace corresponding to eigenvalue 1 may not equal 1. As a result, the eigenvector will not be unique. The linear combination of two eigenvectors corresponding to the same eigenvalue will still be an eigenvector of the matrix, so there will be infinitely many solutions to our system. This means there are infinite ways to rank the pages. And another important consideration is whether the eigenvector has all positive or all negative components. If some of the components are negative and some of the components are positive, then we can not rank the pages based on this eigenvector. That is because we cannot expect the page to become less important when it receives one more link from some other page.

There are some nice properties for the positive matrix (Definition 3), which guarantees that the dimension of the eigenspace corresponding to eigenvalue 1 is equal to 1 , and the components of the eigenvector are all positive or all negative.

Definition 3. A matrix $A$ is positive if all the entries $A_{i j}>0$.

Theorem 5.1.2. If matrix $A$ is positive and is a column Markov matrix, then the dimension of the eigenspace corresponding to eigenvalue 1 is equal to 1 and the entries of the eigenvector are all positive or all negative.

The proof for Theorem 5.1.2 can be found in [6].

A modification to the matrix $A$ can make it to be a positive column Markov matrix. Then the solution exists and is unique. Let $M$ represent the modified matrix which is defined in the following way:

$$
M=(1-m) A+m S
$$

where $m(0<m<1)$ is a damping factor, and $\mathrm{S}$ is an $n \times n$ matrix with all entries equal to $\frac{1}{n}$. The meaning of this formula is that one person may stop clicking the link on one page, but restart surfing in the Internet at a random page with a probability $m$. Or if we think of it in the context of voting, then it means that everybody will 
send an additional $\frac{m}{n}$ vote to all the others. If the vote is sent from node $i$ with score $x_{i}$, then by combining with the score, the vote is equal to $\frac{m}{n} x_{i}$. Usually, $m$ is chosen be 0.15. For the example given in Figure 5.1, we find that

$$
\begin{aligned}
M & =(1-0.15)\left(\begin{array}{cccc}
0 & 1 & \frac{1}{2} & 0 \\
\frac{1}{3} & 0 & 0 & \frac{1}{2} \\
\frac{1}{3} & 0 & 0 & \frac{1}{2} \\
\frac{1}{3} & 0 & \frac{1}{2} & 0
\end{array}\right)+0.15\left(\begin{array}{cccc}
\frac{1}{4} & \frac{1}{4} & \frac{1}{4} & \frac{1}{4} \\
\frac{1}{4} & \frac{1}{4} & \frac{1}{4} & \frac{1}{4} \\
\frac{1}{4} & \frac{1}{4} & \frac{1}{4} & \frac{1}{4} \\
\frac{1}{4} & \frac{1}{4} & \frac{1}{4} & \frac{1}{4}
\end{array}\right) \\
& =\left(\begin{array}{cccc}
0.0375 & 0.8875 & 0.4625 & 0.0375 \\
0.3208 & 0.0375 & 0.0375 & 0.4625 \\
0.3208 & 0.0375 & 0.0375 & 0.4625 \\
0.3208 & 0.0375 & 0.4625 & 0.0375
\end{array}\right)
\end{aligned}
$$

Now $M$ is a positive column Markov matrix, and possesses the property stated in Theorem 5.1.2. We find that the eigenspace corresponding to eigenvalue 1 has dimension 1 . The eigenvector is

$$
\left(\begin{array}{l}
0.6397 \\
0.4438 \\
0.4438 \\
0.4438
\end{array}\right)
$$

Still node 1 is the most important one.

\subsection{PageRank Applied to Swarms}

In Chapter 2, from the simulation we saw that there is information transfer between individuals, since we just tell the leaders to go to the right, and the whole swarm went right eventually. In Chapter 4, we used Local Transfer Entropy to quantify this information. The information transferred between two individuals works like a link between two individuals, and this link is a directional link from the sender to the receiver. If we rank the transfer entropy between each pair in the swarm, we can 
choose the top pairs using a TE threshold, and different individuals may have different incoming and outgoing links. Based on these links, we could apply the PageRank method.

\subsection{Method}

Based on the three-zone model introduced in Chapter 1, we could get the velocity and position for each individual at each time step. In Chapter 4, we find that the transfer entropy will damp to zero when the swarm gets to a steady state. If the external information evolves with time, then the swarm will reorganize and evolve with time. Using this procedure, we can measure TE and the underlying network as a function of time.

When we calculate the transfer entropy, we need the probability distribution function(PDF). In Chapter 4, we treat the individuals differently based on the roles of the receiver. This time we treat them the same when we calculate PDF. In Chapter 4, we divide the value of the variables which we are interested in into several bins, and then calculate the PDF based on these bins. This time, we simplify our calculation. We just consider whether the velocity is changing or not by setting a threshold for the acceleration. In our problem, it will be the difference of the velocity between two adjacent time steps. If the acceleration for individual $i$ is less than the threshold at time step $n$, then we label 0 for $i$ at time $n$. If the acceleration for individual $i$ is greater than the threshold, then we label 1 for $i$ at time $n$.

$$
x_{n}= \begin{cases}1 & \text { if } \vec{v}_{n+1}-\vec{v}_{n} \geq \text { TE threshold } \\ 0 & \text { if } \vec{v}_{n+1}-\vec{v}_{n}<\text { TE threshold }\end{cases}
$$

Thus, each individual in the swarm could be labelled by a series of number consist of 0 and 1. The process could be found in Figure 5.2. The transfer entropy is calculated based on these $0 \mathrm{~s}$ and $1 \mathrm{~s}$ using the formula (4.21). Here we just consider the transfer entropy between each pair. During the calculation, we neglect the existence of other individuals. That means we do not condition on the status of other individuals. Since 


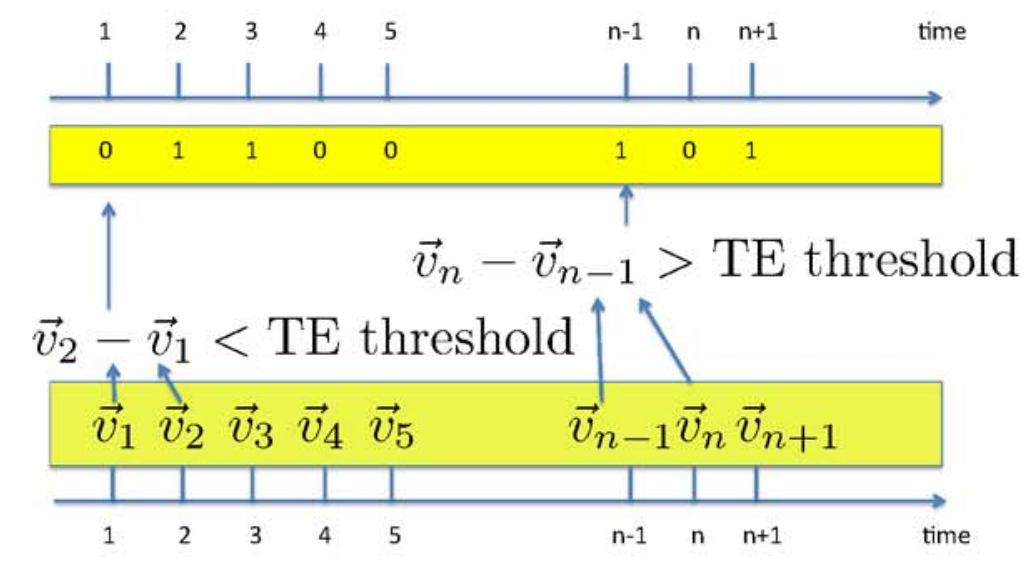

Figure 5.2: The process of creating binary data for individual $i$

we already binary our data, each individual is represented by a binary signal. When using the formula (4.21), we will also neglect the term $W$ which represents the current speed. If we just consider a history of $1 \mathrm{step}$, then $k=1$. So finally, the formula we use is as follows:

$$
\begin{aligned}
t_{Y \rightarrow X}(n+1) & =\log _{2} \frac{p\left(x_{n+1} \mid x_{n}, y_{n}\right)}{p\left(x_{n+1} \mid x_{n}\right)} \\
& =\log _{2} \frac{p\left(x_{n+1}, x_{n}, y_{n}\right) p\left(x_{n}\right)}{p\left(x_{n+1}, x_{n}\right) p\left(x_{n}, y_{n}\right)}
\end{aligned}
$$

where $x_{n} \in\{0,1\}, y_{n} \in\{0,1\}$ for $n=1 \ldots \infty$.

For each pair $i$ and $j$, we could get the local transfer entropy $t_{i j}^{n}$ from $j$ to $i$ at each time step $\mathrm{n}$. Sum the local transfer entropy $t_{i j}^{n}$ over the time, then we could get the total entropy $T_{i j}$ transferred from $j$ to $i$. Rank $T_{i j}$ from the highest to the lowest and choose the top $k$ to build our Column Markov Matrix $A$. If $T_{i j}$ is among the top $k$ values, then $A_{i j}=1$, else $A_{i j}=0$. We choose $k$ in the way such that any $j$ exists at least once and $k$ is the smallest number among all the possible $k$. The way we choose $k$ is to make sure that every column sum of $A$ is non-zero, and it will not add unnecessary links into the network. The column sum is non-zero, then we could 
normalize each column to make the column sum of $A$ equal to 1 , so that $A$ is a Column Markov Matrix. Adding unnecessary links into our network will make the difference between the followers and leaders more vagueness. For example, if we choose all the local transfer entropy to make matrix $A$, then after normalization, $A$ is a Matrix with all entries equal to each other. There is no difference between the followers and the leaders. As a result, we cannot figure out who are the leaders and who are the followers. After we get the Column Markov Matrix A, we could apply the modified PageRank method. This suggest the following algorithm for determining leaders in a swarm:

1. Record the velocity for each individual at each time step.

2. Create binary data based on the acceleration of each individual. The length of the series for each individual is equal to the total number of time steps.

3. Calculate the PDF between each pair and using the formula (5.3) to calculate the local transfer entropy. We need to calculate the local transfer entropy $t_{j i}^{n}$ from $i$ to $j$. We also need to calculate the local transfer entropy $t_{i j}^{n}$ from $j$ to $i$.

4. Sum over the local transfer entropy $t_{i j}^{n}$ along the time to get the total transfer entropy $T_{i j}$.

5. Choose suitable $k$, and based on the selected $T_{i j}$ to make the Column Markov Matrix $A$.

6. Find the eigenspace of $A$ corresponding to eigenvalue 1 . If the eigenspace corresponding eigenvalue 1 is not 1 dimension. Add a damping factor $m$, to make $A$ to be a positive column Markov Matrix $M$ using the formula (5.2).

7. Calculate the eigenvector $v_{1}$ of $M$ corresponding the eigenvalue 1 .

8. Normalize $v_{1}$ to make the sum of all the entries in $v_{1}$ is equal to 1 .

9. Based on the entries of $v_{1}$ to find the leader.(Details are listed in section 5.4.1)

\subsection{Experiments}

The difference between the Couzin model and our model is that in the Couzin model the desired velocity of the leader is a linear combination of the local interaction and the external information, which means the coefficient ahead of the external information is a constant. In our model, we introduce a nonlinear term so that the leader 
will respond to the external information based on the local density. First, we perform the experiment on Couzin model so that we could control the weight that the leader respond to the external information to see how the weight affects the result.

\subsubsection{Couzin Model}

In Couzin model, the desired velocity of the leader is defined by the following equation(2.15):

$$
\vec{v}_{l d}=(1-\alpha)\left[H_{\sigma_{1}} *\left(\rho_{f}+\rho_{l}\right)+\frac{G_{\sigma_{2}} *\left(\rho_{f} \vec{v}_{f}+\rho_{l} \vec{v}_{l}\right)}{G_{\sigma_{2}} *\left(\rho_{f}+\rho_{l}\right)}+c_{a} K_{\sigma_{3}} *\left(\rho_{f}+\rho_{l}\right)\right]+\alpha \vec{g}
$$

Usually, $\alpha$ is chosen to be 0.5. So we perform the simulation with $\alpha=0.5$. Other parameters are the same as those in the simulation performed in Chapter 2. We place 100 individuals on a $10 \times 10$ lattice with $15 \%$ random selected leaders. The initial speed is 1 but the direction is randomly chosen. Then we use the model (2.15) to perform the simulation. The simulation last 3200 time steps. We change the external information $\vec{g}$ every 800 steps from $(0,1)$ to $\left(\frac{\sqrt{2}}{2}, \frac{\sqrt{2}}{2}\right),(1,0),\left(-\frac{\sqrt{2}}{2}, \frac{\sqrt{2}}{2}\right)$.

The movement at key time step is shown in Figure 5.3. The red arrows represent the randomly chosen leaders. We will use it to test our PageRank algorithm to see whether or not it could find the leaders.

Following the steps stated in section 5.3, we find that the eigenspace corresponding to eigenvalue 1 of matrix $A$ has dimension 1, so we did not add any damping factor. We plot the normalized eigenvector $\vec{v}_{1}$ in increasing order and mark the position of the leaders with '*' and dashed lines. We define the value of the element $v_{1 i}$ in $\vec{v}_{1}$ as the score of the individual $i$.

From Figure 5.4, we note that most of the leaders have a relatively small score. Examining the smallest entries in the eigenvector, we obtain Figure 5.5. From Figure 5.5, we find that 13 out of 15 leaders are located before the jump in value. We could use this jump to estimate the amount of leaders and locate the leaders. So here is our proposition: 

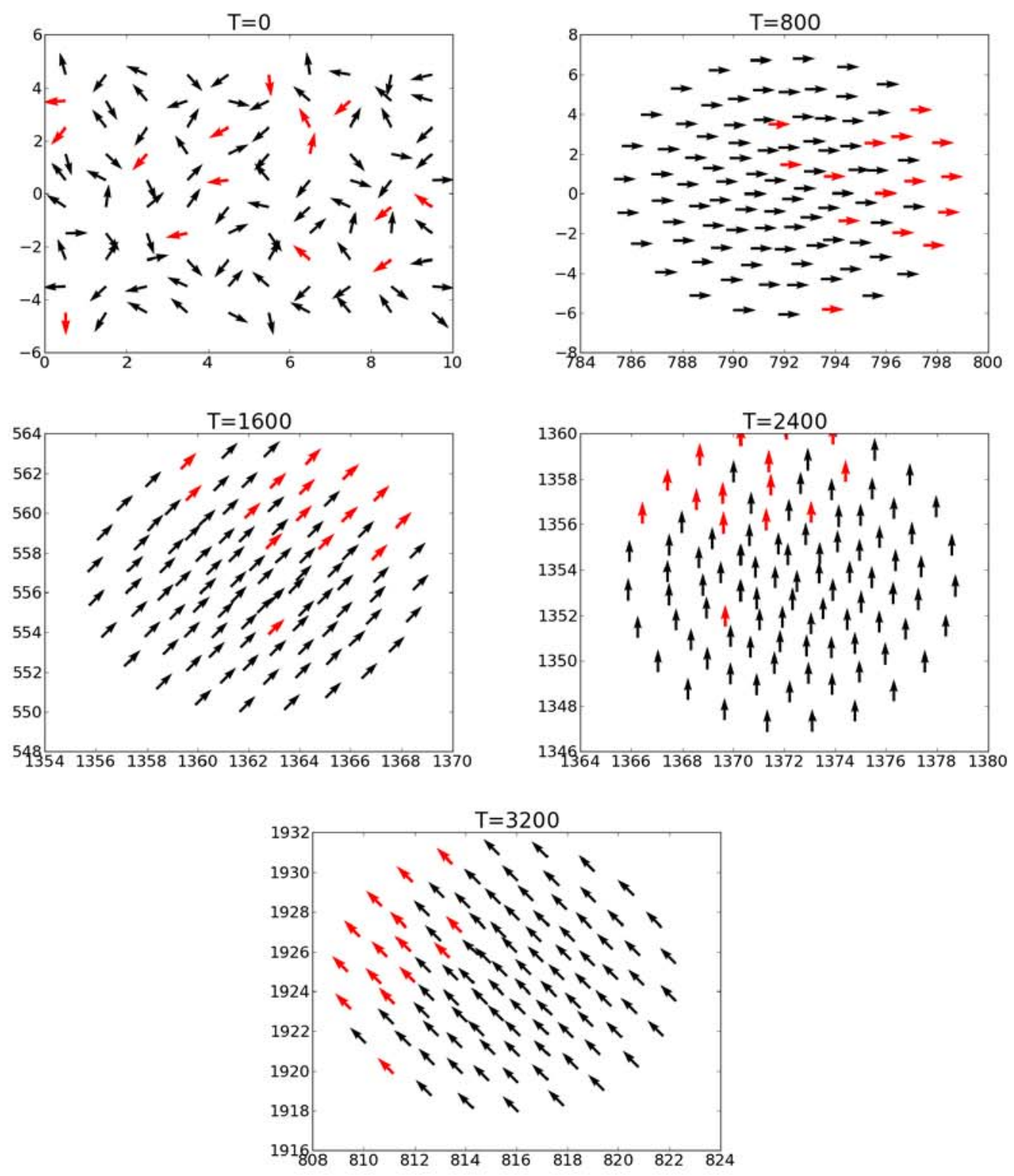

Figure 5.3: Movement at key time step

Proposition 5.4.1. The leaders are below the second jump in the first derivative of the vector consisting of all the entries of the sorted and normalized eigenvector corresponding to eigenvalue 1.

We will perform several additional experiments to test our proposition. We use the same parameters as the ones we used in last simulation. But we choose different groups of leaders in each experiment and we set a different initial velocity but speed is 


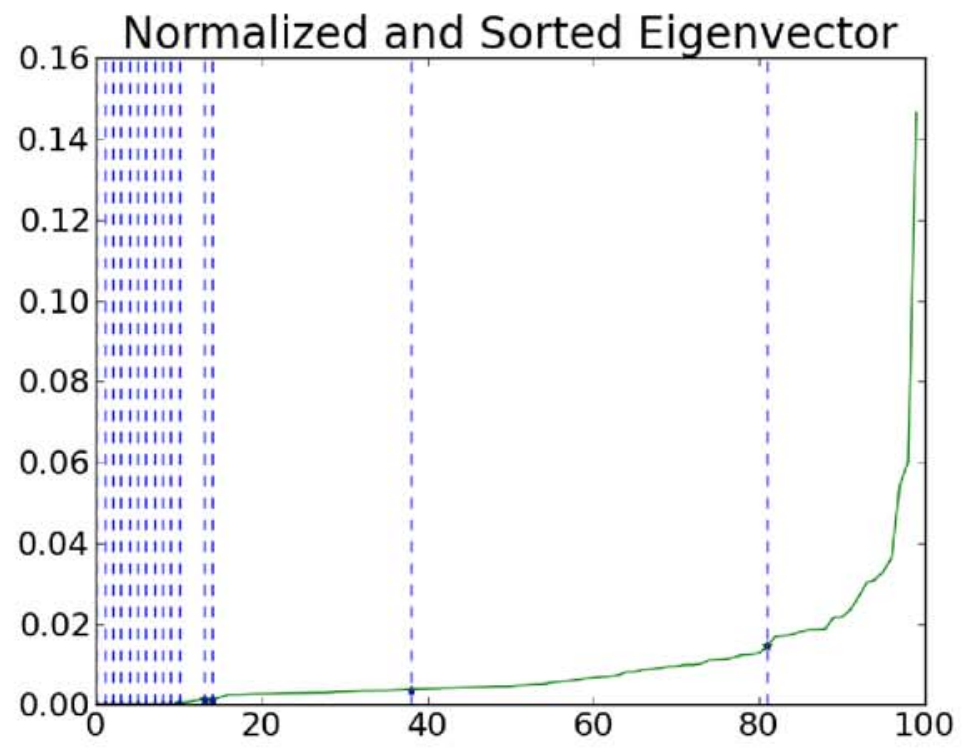

Figure 5.4: Leader's index position in the sorted eigenvector

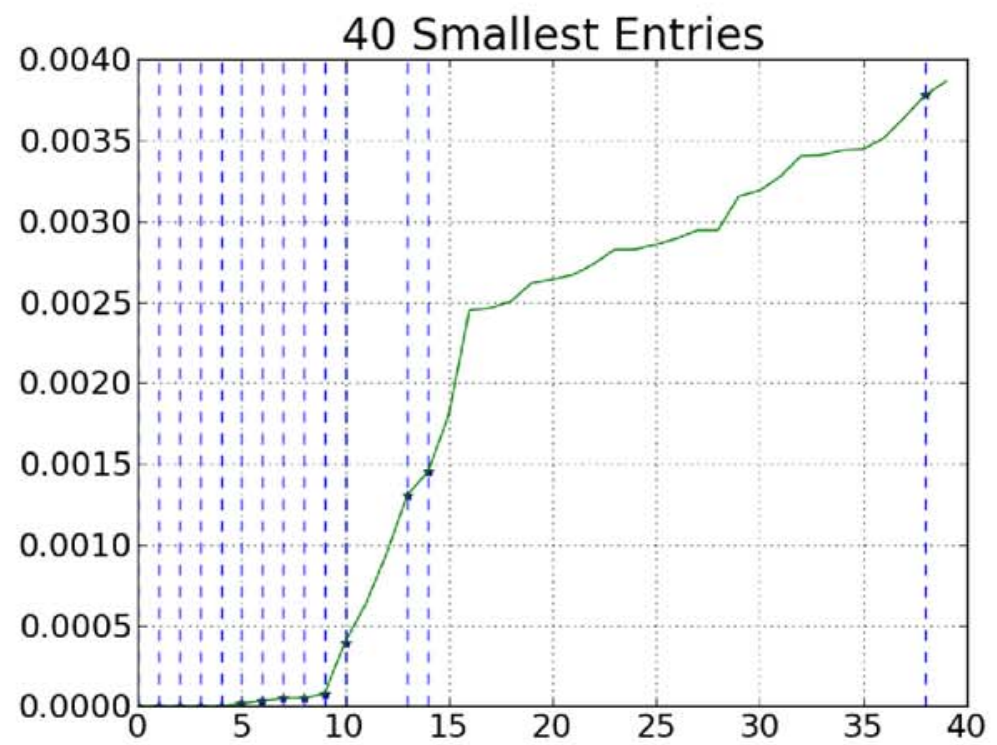

Figure 5.5: Leader's index position in the sorted eigenvector

still equal to 1 . We use $N_{\text {est }}$ to represent the number of leaders in the swarm estimated by the algorithm. $N_{\text {find }}$ represents the number of actual leaders found by the algorithm. $N_{\text {true }}$ represents the number of leaders in the swarm (15 in our experiment). Our result is shown in Table 5.1. This result tells us that the algorithm in section 5.3 can effectively 


\begin{tabular}{|c|c|c|c|c|c|c|c|c|}
\hline \hline Experiment & 1 & 2 & 3 & 4 & 5 & 6 & 7 & 8 \\
\hline$N_{\text {est }}$ & 15 & 12 & 14 & 13 & 14 & 14 & 18 & 14 \\
\hline$N_{\text {find }}$ & 14 & 12 & 13 & 13 & 14 & 14 & 13 & 13 \\
\hline $\begin{array}{c}N_{\text {find }} \\
N_{\text {est }}\end{array}$ & $92.86 \%$ & $93.33 \%$ & $100 \%$ & $92.86 \%$ & $100 \%$ & $100 \%$ & $100 \%$ & $72.22 \%$ \\
\hline$\frac{N_{\text {find }}}{N_{\text {true }}}$ & $86.67 \%$ & $93.33 \%$ & $80 \%$ & $86.67 \%$ & $86.67 \%$ & $93.33 \%$ & $93.33 \%$ & $86.67 \%$ \\
\hline \hline Experiment & 9 & 10 & 11 & 12 & 13 & 14 & Average & \\
\hline$N_{\text {est }}$ & 15 & 13 & 19 & 11 & 14 & 17 & 14.5 & \\
\hline$N_{\text {find }}$ & 14 & 12 & 14 & 11 & 13 & 13 & 13.07 & \\
\hline$\frac{N_{\text {find }}}{N_{\text {est }}}$ & $93.33 \%$ & $92.31 \%$ & $73.68 \%$ & $100 \%$ & $92.86 \%$ & $76.47 \%$ & $91.42 \%$ & \\
\hline$\frac{N_{\text {find }}}{N_{\text {true }}}$ & $93.33 \%$ & $80 \%$ & $93.33 \%$ & $73.33 \%$ & $86.67 \%$ & $86.67 \%$ & $87.14 \%$ & \\
\hline \hline
\end{tabular}

Table 5.1: Results of PageRank method for finding leaders. 14 experiments are performed with the same parameters except the initial orientation and leaders are randomly chosen.

identify leaders. $91.42 \%$ of these possible leaders are true leaders. Additionally, these true leaders compose $87.14 \%$ of the whole leader group.

\subsubsection{The Weight of the External Information}

In our model, the leaders respond to the external information differently based on the local density. So, we would like to know how the algorithm works with different weight of external information. Here we perform some experiments with different $\alpha$ in model (5.4). We choose $\alpha$ from 0.1 to 0.45 incrementing by 0.05 each time. We find that the algorithm also works well. Results are shown in Table 5.2. Decreasing $\alpha$ to the level of $10^{-2}$, the results are shown in Table 5.3. From the result, we find that when $\alpha=0.01$, we could only find less than half of the leaders. We would like to know whether this phenomena is special or a general case. So, we decrease $\alpha$ to the level of $10^{-3}$. When $\alpha=0.009$, the graph for the sorted and normalized eigenvector in shown in the Figure 5.6. We find that half of the leaders have a relatively high score. When $\alpha=0.001$, the result is shown in Figure 5.7. The leaders are almost scattered 


\begin{tabular}{|c|c|c|c|c|c|c|c|c|}
\hline \hline$\alpha$ & 0.1 & 0.15 & 0.2 & 0.25 & 0.3 & 0.35 & 0.4 & 0.45 \\
\hline$N_{\text {est }}$ & 12 & 14 & 15 & 20 & 12 & 13 & 12 & 11 \\
\hline$N_{\text {find }}$ & 12 & 14 & 14 & 15 & 11 & 13 & 11 & 10 \\
\hline$\frac{N_{\text {find }}}{N_{\text {est }}}$ & $100 \%$ & $100 \%$ & $93.33 \%$ & $75 \%$ & $91.67 \%$ & $100 \%$ & $91.67 \%$ & $90.91 \%$ \\
\hline$\frac{N_{\text {find }}}{N_{\text {true }}}$ & $80 \%$ & $93.33 \%$ & $93.33 \%$ & $100 \%$ & $73.33 \%$ & $86.67 \%$ & $73.33 \%$ & $66.67 \%$ \\
\hline \hline
\end{tabular}

Table 5.2: Results for $\alpha$ in $O\left(10^{-1}\right)$

\begin{tabular}{|c|c|c|c|c|c|c|c|c|c|}
\hline \hline$\alpha$ & 0.01 & 0.02 & 0.03 & 0.04 & 0.05 & 0.06 & 0.07 & 0.08 & 0.09 \\
\hline$N_{\text {est }}$ & 12 & 11 & 14 & 15 & 14 & 13 & 13 & 14 & 13 \\
\hline$N_{\text {find }}$ & 7 & 10 & 13 & 14 & 13 & 13 & 13 & 13 & 13 \\
\hline$\frac{N_{\text {find }}}{N_{\text {est }}}$ & $58.33 \%$ & $90.91 \%$ & $92.86 \%$ & $93.33 \%$ & $92.86 \%$ & $100 \%$ & $100 \%$ & $92.86 \%$ & $100 \%$ \\
\hline$\frac{N_{\text {find }}}{N_{\text {true }}}$ & $46.67 \%$ & $66.67 \%$ & $86.67 \%$ & $93.33 \%$ & $86.67 \%$ & $86.67 \%$ & $86.67 \%$ & $86.67 \%$ & $86.67 \%$ \\
\hline \hline
\end{tabular}

Table 5.3: Results for $\alpha$ in $O\left(10^{-2}\right)$

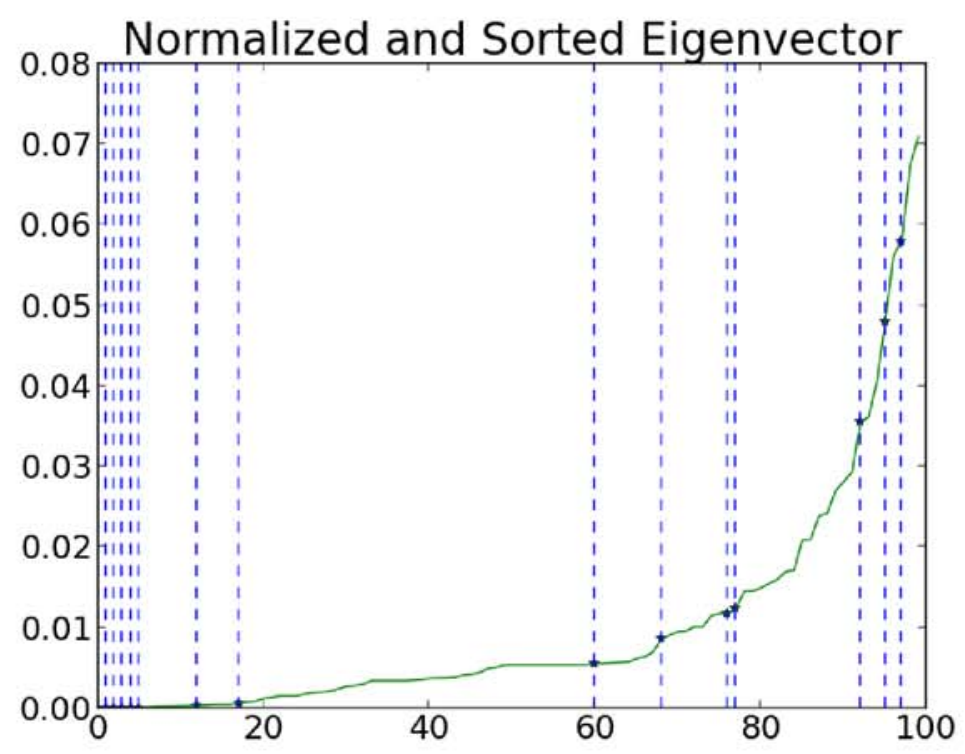

Figure 5.6: Leader's index position in the sorted eigenvector for $\alpha=0.009$ 


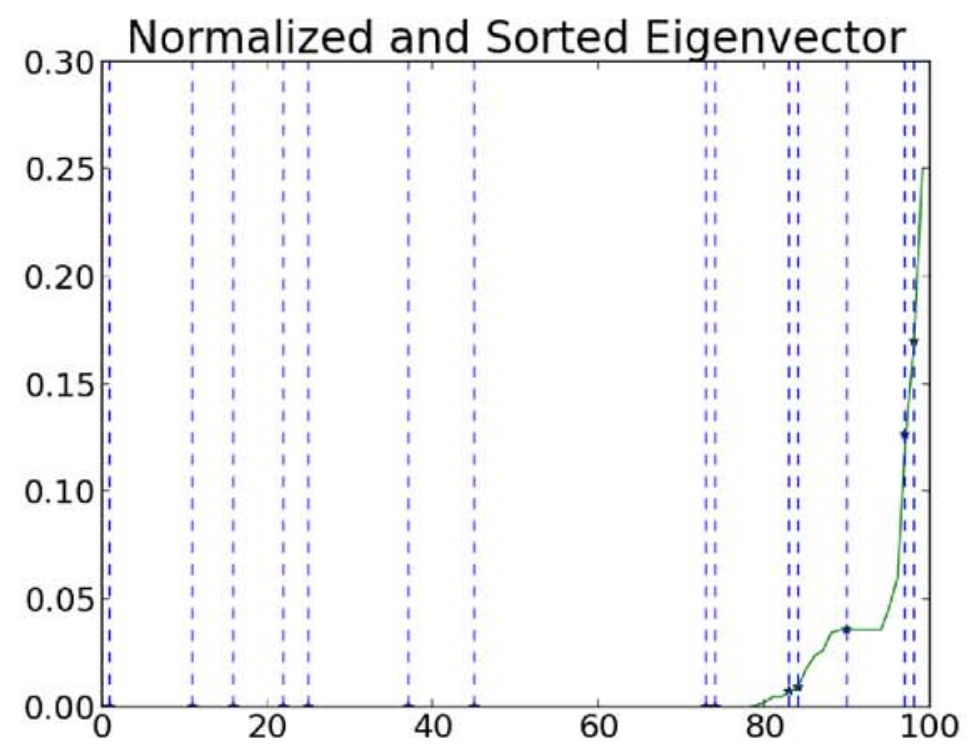

Figure 5.7: Leader's index position in the sorted eigenvector for $\alpha=0.001$

randomly. If we take a further look at the 40 smallest entries in the eigenvector, we find that almost all of them are equal to 0 . So there is no difference between the leaders and the followers from the score estimated by the normalized eigenvector. If we decrease $\alpha$

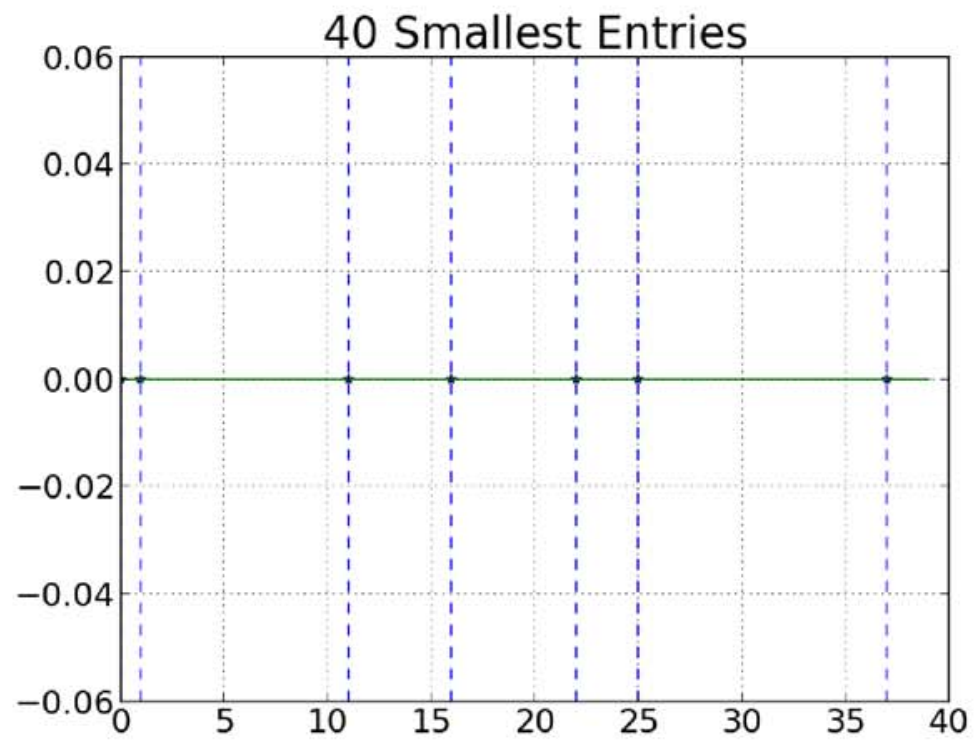

Figure 5.8: Leader's index position in the 40 smallest entires of the sorted eigenvector for $\alpha=0.001$. 
further, the leaders just randomly scattered. For example, when $\alpha=0.0005$, we obtain Figure 5.9. So this method only works for $\alpha$ in the level of $10^{-2}$ or above.

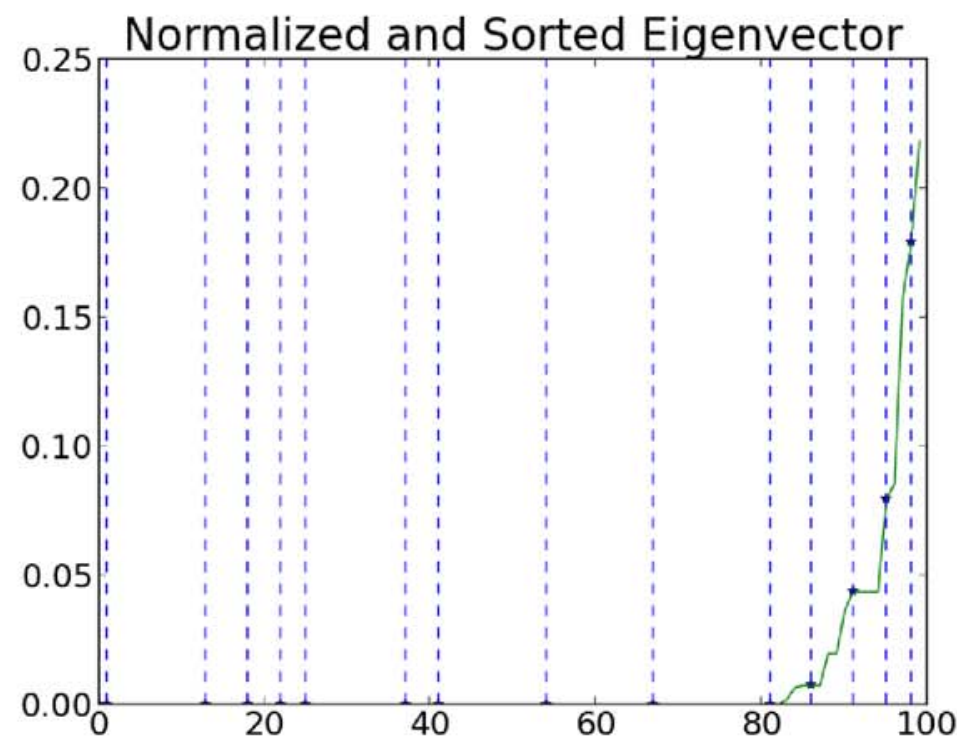

Figure 5.9: Leader's index position in the sorted eigenvector for $\alpha=0.0005$.

\subsubsection{Our model}

We would like to test whether the algorithm will work for our model. We choose the same group of leaders as the ones that were chosen for the simulation in Couzin model. We find that the leaders are almost randomly scattered in the normalized eigenvector as shown in Figure 5.10. In order to find the reason, we look at the coefficient before the external information along the time. The result is shown in Figure 5.11. From the graph, we find that the weight is below $10^{-2}$ except leader 49, so the leaders are randomly scattered in the eigenvector due to the limitation of the PageRank Method.

Take a look at the configurations of the swarms at key time steps (Figure 5.12). We find that local density around leader 49 is relatively low, where leaders respond to the external information strongly. 


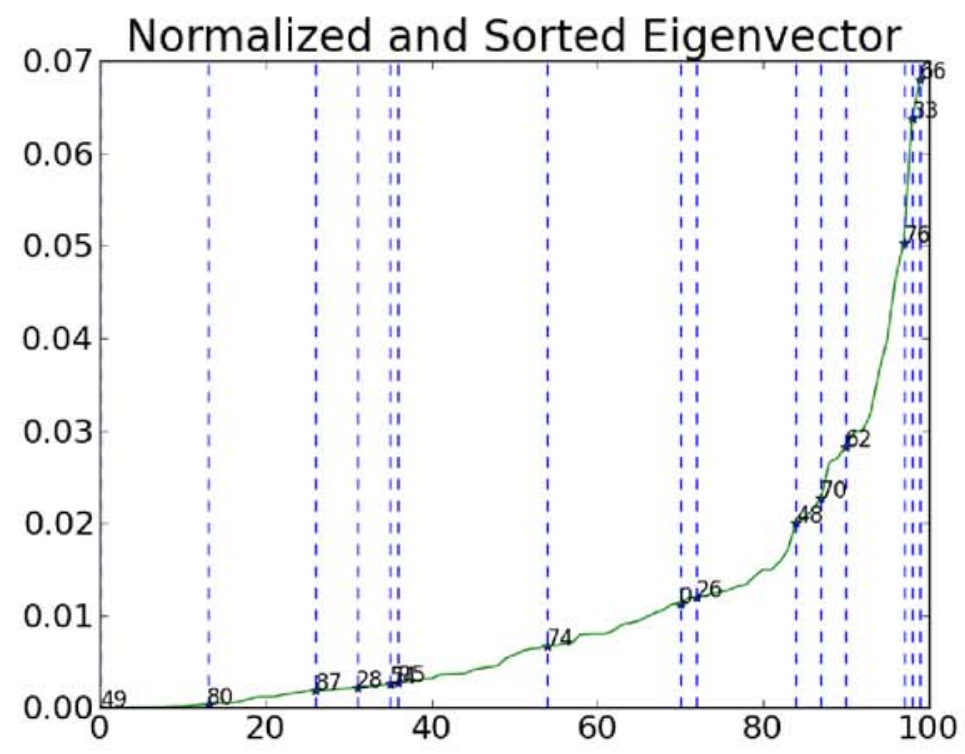

Figure 5.10: Leader's index position in the sorted eigenvector for our model.

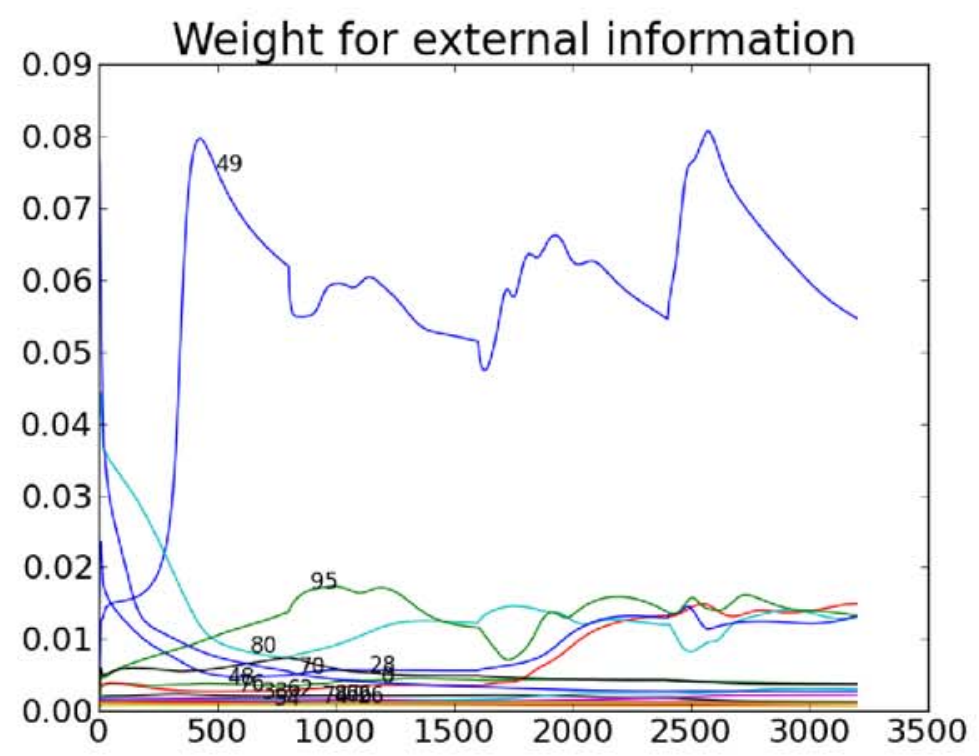

Figure 5.11: Weight before the external information for leaders along the time

\subsection{Conclusion}

In this chapter, we explore a method to find the leaders in the swarm. Due to the knowledge from Chapter 4, we could detect the difference between the leaders 

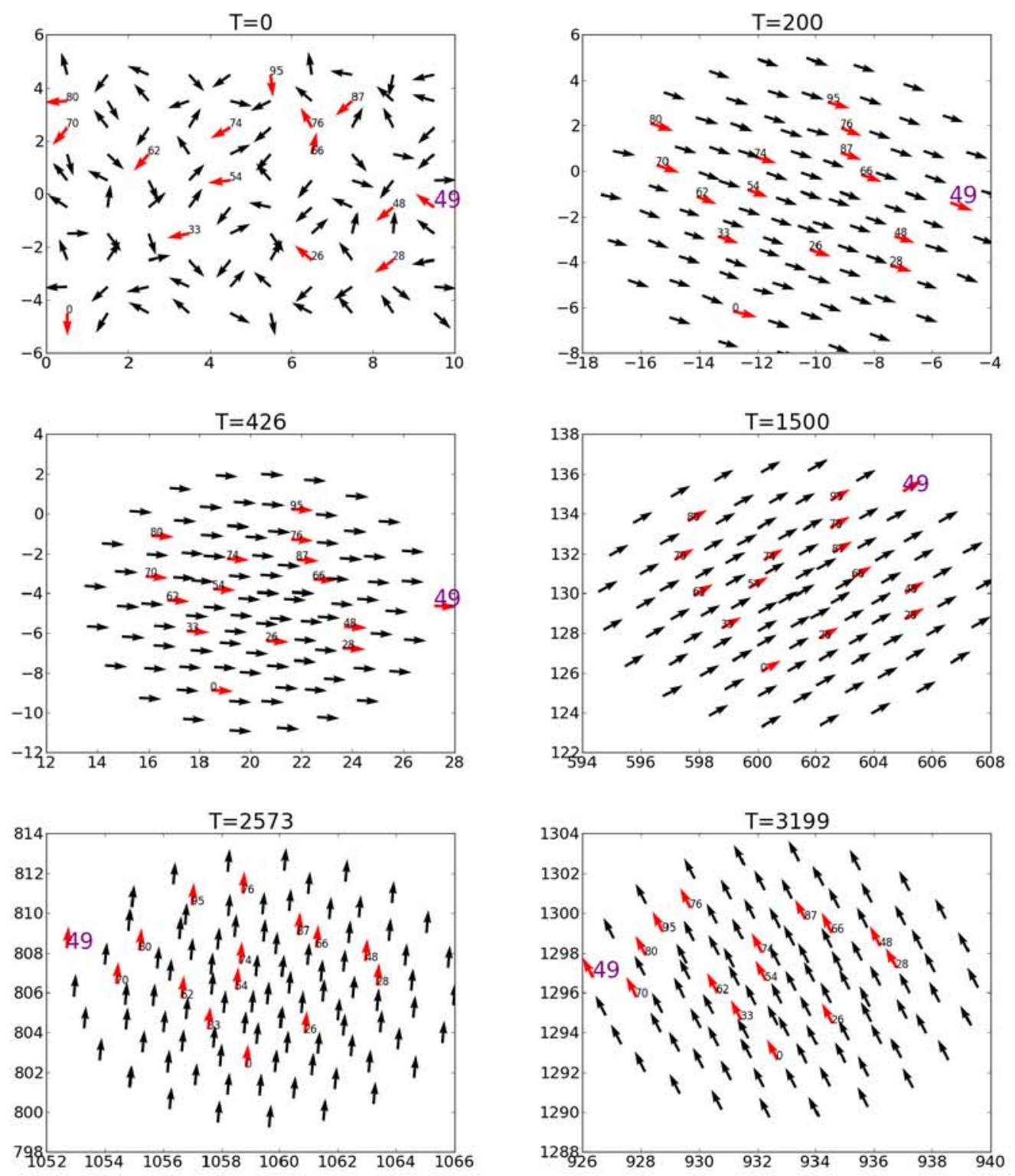

Figure 5.12: Leader 49's movement at key time step

and the followers through transfer entropy. But the limitation is that we separate the groups based on the roles of the receivers firstly, and then calculate the transfer entropy separately for leaders and for followers. Finally, we find the leaders receive less transfer entropy than the followers. In this chapter, we still use transfer entropy to quantify the properties for leaders and followers, but we treat them the same at the beginning when we calculate the transfer entropy. We cannot find the leaders directly from the value 
of the transfer entropy, like ranking the values and then choosing the smallest one to be the leaders or using some cluster methods, so we try other methods. We build a network based on the value of the transfer entropy. Inspired by the network, we apply the PageRank method, which is used by Google to rank the importance of web pages, to locate the leaders in the swarm. We make a small change to this method. In Google search engine, the pages with large scores are ranked higher. But in our algorithm, we choose the ones with lower scores as our leaders. This method could effectively find the leaders if the leaders respond to the external information strongly enough. More exactly, in linear model or Couzin model, the weight before the external information should be greater than $O\left(10^{-2}\right)$. We test the method in our nonlinear model. Since the weight is changing along the time due to the change of the local density, we record the weight before the external information along the time for the leaders. We find that the weight is lower than $O\left(10^{-2}\right)$. So the PageRank method could not find the leaders in our model. Performing the algorithm on our model also shows the same result. From this point of view, we can say that our nonlinear model is really covert.

So far, in this thesis, we introduce a new nonlinear model for large swarms based on the three-zone interactions. Compared with the linear model, the leaders in our model are embedded in the swarm instead of accumulating into the front of the group. We perform stability analysis on the nonlinear model, and find that the stability criteria is as the same as the leaderless model. It tells us that we could inject external information into the system without changing the stability criteria. We verify our analysis result both on ideal platform and QualNet. We also perform several experiments with two group of leaders possessing different external information. The simulation results tell us that the group will follow the average direction when the information differential is small, and the group will randomly choose a direction of the two options if the information differential is large. In order to know when the bifurcation happens, we perform two case studies, which is a simplified form of our nonlinear model. Then we consider a reverse problem: When we observe a group of individuals in motion, is it possible to distinguish the leaders from the followers? If so, 
how do we find the leaders in the swarm. We find that the leaders receive less transfer entropy than the followers. So we could find the difference between the leaders and the followers. Then based on the transfer entropy, we could build a network among all the individuals in the group. We apply the PageRank Method to find the leaders embedded in the swarms. This method could effectively find the leaders if the leaders respond to the external information strong enough. For our model, since the weight before the external information is so small that the leaders are difficult to locate. From this point of view, the leader in our model is really covert.

There are still some open questions that could be done in future. We may apply the PageRank Method to real world data, such as the birds or fish, to see whether we could figure out who are the leaders and who are the followers. We could continue in searching different methods to see whether we could find the leaders in the simulation results performed from our model. There are many connectivity-based ranking algorithms besides PageRank, like HITS [17] and OPIC [1]. We could test those methods on our model to see the output. 


\section{BIBLIOGRAPHY}

[1] Serge Abiteboul, Mihai Preda, and Gregory Cobena. Adaptive on-line page importance computation. In Proceedings of the twelfth international conference on World Wide Web, pages 280-290, 2003.

[2] Rachid Alami, Raja Chatila, and Hajime Asama. Distributed Autonomous Robotic Systems 6. Springer Japan, Tokyo, 2007.

[3] M Andersson and J Wallander. Kin selection and reciprocity in flight formation? Behav. Ecol., 15:158-162, 2003.

[4] I Aoki. A simulation study on the schooling mechanism in fish. Bulletin of the Japanese Society of Scientific Fisheries, 48:1081-1088, 1982.

[5] S. Brin and L. Page. The anatomy of a large-scale hypertextual Web search engine. Computer Networks and ISDN Systems, 1998.

[6] Kurt Bryan and Tanya Leise. The $\$ 25,000,000,000$ eigenvector: The linear algebra behind google. SIAM Review, 48(3):569-581, 2006.

[7] J Buhl, D J T Sumpter, I D Couzin, J J Hale, E Despland, E R Miller, and S J Simpson. From disorder to order in marching locusts. Science (New York, N.Y.), 312(5778):1402-6, June 2006.

[8] A. Caruso, F. Paparella, L. F. M. Vieira, M. Erol, and M. Gerla. The Meandering Current Mobility Model and its Impact on Underwater Mobile Sensor Networks. 2008 IEEE INFOCOM - The 27th Conference on Computer Communications, pages 221-225, April 2008.

[9] Iain D Couzin. Self-Organization and Collective Behavior in Vertebrates. 32:1-75, 2003.

[10] Iain D Couzin, Jens Krause, Nigel R Franks, and Simon A Levin. Effective leadership and decision-making in animal groups on the move. Nature, 433(7025):513-6, February 2005.

[11] ID Couzin, J Krause, R James, GD Ruxton, and N Franks. Collective memory and spatial sorting in animal groups. Journal of Theoretical Biology, 218:1-11, 2002. 
[12] Thomas M. Cover and Joy A. Thomas. Elements of infomation theory. John Wiley \& Sons, Inc., 2006.

[13] Deboran M. Gordon, Richard E. Paul, and Karen Thorpe. What is the function of encounter patterns in ant colonies? Animal Behavior, 45:1083-1100, 1993.

[14] A Huth and C Wissel. The simulation of the movement of fish schools. J. theor. Biol., 156:365-385, 1992.

[15] Xu J, Liu ZR, Liu R, and Yang QF. Information transmission in human cerebral information transmission in human cerebral cortex. Physica D, 106:363-374, 1997.

[16] Graeme D. Ruxton Jens Krause. Living in Groups. Oxford University Press, 2002.

[17] J. M. Kleinberg. Authoritative sources in a hyperlinked environment. Journal of ACM, 46(5):604-632, September 1999.

[18] Joseph T. Lizier, Mikhail Prokopenko, and Albert Y. Zomaya. Local information transfer as a spatiotemporal filter for complex systems. Physical Review E, $77(2): 026110,2008$.

[19] Ryan Lukeman, Yue-Xian Li, and Leah Edelstein-Keshet. Inferring individual rules from collective behavior. Proceedings of the National Academy of Sciences of the United States of America, 107(28):12576-12580, June 2010.

[20] Jennifer M Miller. A Whole Greater Than the Sum of its Parts: Mathematically Modeling and Analyzing Swarms. PhD thesis, University of Delaware, 2012.

[21] Jennifer M Miller, Allison Kolpas, Juchem Neto, Joao Plinio, and Louis F Rossi. A continuum three-zone model for swarms. Bulletin of Mathematical Biology, 74(3):536-61, March 2012.

[22] Jennifer M. Miller, X. Rosalind Wang, Joseph T. Lizier, Mikhail Prokopenko, and Louis F. Rossi. Measuring information dynamics in swarms. In Mikhail Prokopenko, editor, Guided Self-Organization: Inception, pages 343-364. Springer, 2014.

[23] Máté Nagy, Zsuzsa Akos, Dora Biro, and Tamás Vicsek. Hierarchical group dynamics in pigeon flocks. Nature, 464(7290):890-3, April 2010.

[24] Hiro-Sato Niwa. Power-law versus exponential distributions of animal group sizes. Journal of Theoretical Biology, 224(4):451-457, October 2003.

[25] Hiro-Sato Niwa. Space-irrelevant scaling law for fish school sizes. Journal of theoretical biology, 228(3):347-57, June 2004. 
[26] P. Ogren, E. Fiorelli, and N.E. Leonard. Cooperative Control of Mobile Sensor Networks: Adaptive Gradient Climbing in a Distributed Environment. IEEE Transactions on Automatic Control, 49(8):1292-1302, August 2004.

[27] DA Paley, NE Leonard, Rodolphe Sepulchre, Daniel Grünbaum, and JK Parrish. Spatial Patterns in the Dynamics of Engineered and Biological Networks. IEEE Control Systems Magazine, 27(4):89-105, 2007.

[28] BL Partridge. The structure and function of fish schools. Scientific American, 1982.

[29] MIkhail Prokopenko, Fabio Boschetti, and Alex J. Ryan. An InformationTheoretic Primer on Complexity, Self-Organization, and Emergence. Complexity, 15(1), 2009.

[30] D.V. Radakov. Schooling in the ecology of fish. New York: John Wiley \& Sons., 1973.

[31] H Reuter and B Breckling. Self-organization of fish schools: an object-oriented model. Ecol. Model., 75:147-159, 1994.

[32] Louis Rossi, Xiaofeng Han, and Chien-Chung Shen. Autonomous Navigation of Wireless Robot Swarms with Covert Leaders. The Proceedings of First International Conference on Robot Communication and Coordination, 2007.

[33] Thomas Schreiber. Measuring information transfer. Phys. Rev. Lett., 85(2):461464, July 2000.

[34] G.T. Sibley, M.H. Rahimi, and G.S. Sukhatme. Robomote: a tiny mobile robot platform for large-scale ad-hoc sensor networks. In Proceedings 2002 IEEE International Conference on Robotics and Automation (Cat. No.02CH37292), volume 2, pages 1143-1148. IEEE, 2002.

[35] David Sumpter, Jerome Buhl, Dora Biro, and Iain Couzin. Information transfer in moving animal groups. Theory Biosci, 127(2):177-86, June 2008.

[36] David J. T. Sumpter. Collective animal behavior. Princeton University Press, 2010.

[37] Yu Sun, Louis F. Rossi, Hao Luan, and Chien-Chung Shen. Modeling and Analyzing Large Swarms with Covert Leaders. In 2013 IEEE 7th International Conference on Self-Adaptive and Self-Organizing Systems, pages 169-178. IEEE, September 2013.

[38] J.E. Treherne and W.A. Foster. Group transmission of predator avoidance behaviour in a marine insect: The trafalgar effect. Animal Behaviour, 29(3):911-917, 1981. 
[39] T Vicsek, A Czirok, E Ben-Jacob, I Cohen, and O Shochet. Novel Type of Phase Transition in a System of Self-Driven Particles. Phys. Rev. Lett., 75:1226-1229, 1995.

[40] X Rosalind Wang, Jennifer M Miller, Joseph T Lizier, Mikhail Prokopenko, and Louis F Rossi. Quantifying and tracing information cascades in swarms. PloS ONE, 7(7):e40084, January 2012.

[41] X.Rosalind Wang, Jennifer M. Miller, Jseph T. Lizier, MIkhail Prokopenko, and Louis F. Rossi. Measuring information Storage and Transfer in Swarms. In Proceeedings of the Eleventh European Conference on the Synthesis and Simulation of Living Systems, 2011.

[42] K Warburton and J Lazarus. Tendency-distance models of social cohesion in animal groups. J. theor. Biol., 150:473-488, 1991.

[43] Henri Weimerskirch, Julien Martin, Yannick Clerquin, Peggy Alexandre, and Sarka Jiraskova. Energy saving in flight formation. Nature, 413(6857):697-698, 2001.

[44] Edward O Wilson. Chemical communication among workers of the fire ant. Animal Behaviour, 10:148-158, 1962. 